\title{
ANALYSIS OF THE GHS WEIL DESCENT ATTACK ON THE ECDLP OVER CHARACTERISTIC TWO FINITE FIELDS OF COMPOSITE DEGREE
}

\author{
MARKUS MAURER, ALFRED MENEZES AND EDLYN TESKE
}

\begin{abstract}
In this paper, the authors analyze the Gaudry-Hess-Smart (GHS) Weil descent attack on the elliptic curve discrete logarithm problem (ECDLP) for elliptic curves defined over characteristic two finite fields of composite extension degree. For each such field $\mathbb{F}_{2^{N}}$, $N \in[100,600]$, elliptic curve parameters are identified such that: (i) there should exist a cryptographically interesting elliptic curve $E$ over $\mathbb{F}_{2^{N}}$ with these parameters; and (ii) the GHS attack is more efficient for solving the ECDLP in $E\left(\mathbb{F}_{2^{N}}\right)$ than for solving the ECDLP on any other cryptographically interesting elliptic curve over $\mathbb{F}_{2^{N}}$. The feasibility of the GHS attack on the specific elliptic curves is examined over $\mathbb{F}_{2^{176}}, \mathbb{F}_{2^{208}}, \mathbb{F}_{2^{272}}, \mathbb{F}_{2^{304}}$ and $\mathbb{F}_{2^{368}}$, which are provided as examples in the ANSI X9.62 standard for the elliptic curve signature scheme ECDSA. Finally, several concrete instances are provided of the ECDLP over $\mathbb{F}_{2^{N}}, N$ composite, of increasing difficulty; these resist all previously known attacks, but are within reach of the GHS attack.
\end{abstract}

\section{Introduction}

Let $E$ be an elliptic curve defined over a finite field $K=\mathbb{F}_{2^{N}}$. The elliptic curve discrete logarithm problem (ECDLP) in $E(K)$ is as follows: given $E, P \in E(K), r=\operatorname{ord}(P)$ and $Q \in\langle P\rangle$, find the integer $\lambda \in[0, r-1]$ such that $Q=\lambda P$. We write $\lambda=\log _{P} Q$. The ECDLP is of interest because its apparent intractability forms the basis for the security of elliptic curve cryptographic schemes.

The elliptic curve parameters have to be carefully chosen in order to circumvent some known attacks on the ECDLP. We say that an elliptic curve $E$ over $\mathbb{F}_{2^{N}}$ is cryptographically interesting if: (i) $\# E\left(\mathbb{F}_{2^{N}}\right)$ is almost prime (that is, $\# E\left(\mathbb{F}_{2^{N}}\right)=r d$, where $r$ is prime and $d \in\{2,4\})$ in order to provide maximum resistance to the Pohlig-Hellman [28] and Pollard's rho $[29,26]$ attacks; and (ii) $r$ does not divide $2^{N j}-1$ for each $j \in[1, J]$, where $J$ is large enough so that it is computationally infeasible to find discrete logarithms in $\mathbb{F}_{2^{N J}}$ - in order to resist the Weil pairing [24] and Tate pairing [11] attacks.

Frey [10] first proposed using Weil descent as a means to reduce the ECDLP in elliptic curves over $\mathbb{F}_{2^{N}}$ to the discrete logarithm problem in an abelian variety over a proper subfield $\mathbb{F}_{2^{l}}$ of $\mathbb{F}_{2^{N}}$. Frey's method, which we refer to as the Weil descent attack methodology, was further elaborated by Galbraith and Smart [13]. In 2000, Gaudry, Hess and Smart (GHS) [17] showed how Frey's methodology could be used (in most cases) to reduce any instance of the 
ECDLP to an instance of the discrete logarithm problem in the Jacobian of a hyperelliptic curve over $\mathbb{F}_{2^{l}}$. Since subexponential-time algorithms for the hyperelliptic curve discrete logarithm problem (HCDLP) are known, this could have important implications for the security of elliptic curve cryptographic schemes.

The GHS attack was analyzed in $[17,23]$. It was proven to fail for all cryptographically interesting elliptic curves over $\mathbb{F}_{2^{N}}$, where $N \in[160,600]$ is prime. In other words, the hyperelliptic curves $C$ that are produced either have genus too small (whence $J_{C}\left(\mathbb{F}_{2}\right)$ is too small to yield any non-trivial information about the ECDLP in $E\left(\mathbb{F}_{2^{N}}\right)$ ), or have genus too large $\left(g \geqslant 2^{16}-1\right.$, whence the HCDLP in $J_{C}\left(\mathbb{F}_{2}\right)$ is infeasible). The purpose of this paper is to investigate the applicability of the GHS attack on the ECDLP for cryptographically interesting elliptic curves over $\mathbb{F}_{2^{N}}$ for composite $N \in[100,600]$.

The remainder of this paper is organized as follows. Section 2 provides a brief introduction to the relevant theory of hyperelliptic curves. The GHS Weil descent attack is outlined in Section 3, and an overview of the best methods known for solving the ECDLP and HCDLP is given in Section 4. Our analysis of the applicability of the GHS attack on the ECDLP over characteristic two finite fields of composite extension degree is presented in Section 5. In Section 6, a detailed analysis is presented of the feasibility of the GHS attack on certain elliptic curves specified in the ANSI X9.62 standard. Section 7 lists some ECDLP 'challenges' of increasing difficulty, which should resist all previously known attacks, but which are within reach of the GHS attack. Our conclusions are stated in Section 8.

\section{Hyperelliptic curves}

We provide a brief overview of the theory of hyperelliptic curves, relevant to this paper.

\subsection{Hyperelliptic curves}

Let $k=\mathbb{F}_{q}$ denote the finite field of order $q$. The algebraic closure of $\mathbb{F}_{q}$ is $\bar{k}=$ $\bigcup_{n \geqslant 1} \mathbb{F}_{q^{n}}$. A hyperelliptic curve $C$ of genus $g$ over $k$ is defined by a non-singular equation

$$
v^{2}+h(u) v=f(u)
$$

where $h, f \in k[u], \operatorname{deg} f=2 g+1$, and $\operatorname{deg} h \leqslant g$. Let $L$ be an extension field of $k$. The set of L-rational points on $C$ is $C(L)=\left\{(x, y): x, y \in L, y^{2}+h(x) y=f(x)\right\} \cup\{\infty\}$. The opposite of $P=(x, y) \in C(L)$ is $\widetilde{P}=(x,-y-h(x))$; we also define $\widetilde{\infty}=\infty$. Note that $\widetilde{P} \in C(L)$. Except for the case $g=1$ (since a genus 1 hyperelliptic curve is precisely an elliptic curve), there is no natural group law on the set of points $C(L)$. Instead, one considers the Jacobian of $C$ over $k$, which is a finite group.

\subsection{The Jacobian of a hyperelliptic curve}

The set $D^{0}$ of degree zero divisors of $C$ is the set of formal sums $\sum_{P \in C(\bar{k})} m_{P} P$, where $m_{P} \in \mathbb{Z}, \sum m_{P}=0$, and only a finite number of the values of $m_{P}$ are non-zero. $D^{0}$ is a group under the addition rule

$$
\sum m_{P} P+\sum n_{P} P=\sum\left(m_{P}+n_{P}\right) P
$$

Let $\sigma: \bar{k} \rightarrow \bar{k}$ be the Frobenius map defined by $x \mapsto x^{q}$. The map $\sigma$ extends to $C(\bar{k})$ by $(x, y) \mapsto\left(x^{\sigma}, y^{\sigma}\right)$ and $\infty^{\sigma} \mapsto \infty$, and to $D^{0}$ by $\sum m_{P} P \mapsto \sum m_{P} P^{\sigma}$. The set of zero 
divisors defined over $k$ is

$$
D_{k}^{0}=\left\{D \in D^{0}: D^{\sigma}=D\right\}
$$

The function field of $C$ over $k$, denoted $k(C)$, is the field of fractions of the integral domain of polynomial functions $k[u, v] /\left(v^{2}+h(u) v-f(u)\right)$. For $z \in k(C)$, the divisor of $z$ is $\operatorname{div}(z)=\sum_{P \in C(\bar{k})} v_{P}(z) P$, where $v_{P}(z)$ denotes the multiplicity of $P$ as a root of $z$. Now the set $\operatorname{Prin}_{k}=\{\operatorname{div}(z): z \in k(C)\}$ is a subgroup of $D_{k}^{0}$. The Jacobian of $C$ (over $k$ ) is the quotient group $J_{C}(k)=D_{k}^{0} / \operatorname{Prin}_{k}$.

\subsection{Properties of the Jacobian}

$J_{C}(k)$ is a finite group. A theorem of Weil's implies that

$$
(\sqrt{q}-1)^{2 g} \leqslant \# J_{C}(k) \leqslant(\sqrt{q}+1)^{2 g} .
$$

If $D_{1}$ and $D_{2}$ are in the same equivalence class of divisors in $J_{C}(k)$, we write $D_{1} \sim$ $D_{2}$. Each equivalence class has a unique divisor in reduced form - that is, a divisor $\sum_{P \neq \infty} m_{P} P-\left(\sum_{P \neq \infty} m_{P}\right) \infty$ satisfying: (i) $m_{P} \geqslant 0$ for all $P$; (ii) if $m_{P} \geqslant 1$ and $P \neq \widetilde{P}$, then $m_{\widetilde{P}}=0$; (iii) $m_{P}$ is equal to 0 or 1 if $P=\widetilde{P}$; and (iv) $\sum m_{P} \leqslant g$. Such a reduced divisor $D$ can be uniquely represented by a pair of polynomials $a, b \in k[u]$, where: (i) $\operatorname{deg} b<\operatorname{deg} a \leqslant g$; (ii) $a$ is monic; and (iii) $a \mid\left(b^{2}+b h-f\right)$. We write $D=\operatorname{div}(a, b)$ to mean that $D=\operatorname{gcd}(\operatorname{div}(a), \operatorname{div}(b-v))$, where the gcd of two divisors $\sum_{P \neq \infty} m_{P} P-\left(\sum_{P \neq \infty} m_{P}\right) \infty$ and $\sum_{P \neq \infty} n_{P} P-\left(\sum_{P \neq \infty} n_{P}\right) \infty$ is defined to be $\sum_{P \neq \infty} \min \left(m_{P}, n_{P}\right) P-\left(\sum_{P \neq \infty} \min \left(m_{P}, n_{P}\right)\right) \infty$. The degree of $D$ is $\operatorname{deg} a$. Cantor's algorithm $[4,22]$ can be used to compute the sum of two reduced divisors efficiently, and to express the sum in reduced form.

\subsection{Artin's bound}

In the above, we considered only the imaginary form of a hyperelliptic curve, and not the real form, for which $\operatorname{deg}(f)=2 g+2$ in the defining equation (1). Let $C$ be a hyperelliptic curve (real or imaginary) of genus $g$ over $k=\mathbb{F}_{p}$ with $p$ an odd prime. Then $h=0$ in (1). Artin [3] showed that

$$
\# J_{C}(k)= \begin{cases}\sum_{\nu=0}^{2 g} \tau_{v}, & \text { if } \operatorname{deg} f=2 g+1 \\ -\sum_{\nu=1}^{2 g+1} \nu \tau_{\nu}, & \text { if } \operatorname{deg} f=2 g+2\end{cases}
$$

Here, $\tau_{v}=\sum_{\operatorname{deg} F=v}[f / F]$, where the summation is over all degree-v monic polynomials $F \in \mathbb{F}_{p}[u]$ coprime to $f$, and $[f / F]$ is the polynomial Legendre symbol. We trivially have $\left|\tau_{v}\right| \leqslant p^{\nu}$, and Artin showed that $\left|\tau_{v}\right| \leqslant p^{g}(0 \leqslant v \leqslant 2 g)$ if $\operatorname{deg} f=2 g+1$, and $\tau_{2 g+1}=-p^{g}$ and $\left|\tau_{v}\right| \leqslant 2 p^{g}(1 \leqslant v \leqslant 2 g)$ if $\operatorname{deg} f=2 g+2$. These results can be extended to the case $k=\mathbb{F}_{q}$, where $q=p^{l}$ and $p$ is prime, by replacing the Artin character $\chi(F)=[f / F]$ by the general quadratic character. That is, for a monic irreducible polynomial $F \in \mathbb{F}_{q}[u]$ coprime to $f$, let $\chi(F)$ be equal to 1 or -1 , depending on whether the equation $v^{2}+h(u) v \equiv f(u)(\bmod F(u))$ has a solution $v(\bmod F(u))$, or not. It follows that

$$
\# J_{C}(k) \leqslant \begin{cases}g q^{g}+\sum_{\nu=0}^{g} q^{v}, & \text { if } \operatorname{deg} f=2 g+1 \\ \left((2 g+1)^{2}-g(g+1)\right) q^{g}+\sum_{\nu=1}^{g} v q^{v}, & \text { if } \operatorname{deg} f=2 g+2\end{cases}
$$


Since over constant fields of characteristic two the real case is strictly more general than the imaginary case (see [27]), we work with

$$
B_{2}:=\left((2 g+1)^{2}-g(g+1)\right) q^{g}+\sum_{\nu=1}^{g} v q^{\nu}
$$

as an upper bound on the cardinality of the Jacobian. Notice that the larger $q$ is, the larger is the smallest genus $g$ for which the Artin bound $B_{2}$ is indeed smaller than the Hasse-Weil upper bound

$$
B_{1}:=(\sqrt{q}+1)^{2 g}
$$

\section{Weil descent attack}

Let $l$ and $n$ be positive integers, and let $N=\ln$. Let $q=2^{l}$, and let $k=\mathbb{F}_{q}$ and $K=\mathbb{F}_{q^{n}}$. Consider the (non-supersingular) elliptic curve $E$ defined over $K$ by the equation

$$
E: y^{2}+x y=x^{3}+a x^{2}+b, \quad a \in K, b \in K^{*} .
$$

Gaudry, Hess and Smart [17] showed how Weil descent can be used to reduce the ECDLP in $E(K)$ to a discrete logarithm problem in the Jacobian $J_{C}(k)$ of a hyperelliptic curve $C$ defined over $k$. One first constructs the Weil restriction $W_{E / k}$ of scalars of $E$, which is an $n$-dimensional abelian variety over $k$. Then, $W_{E / k}$ is intersected with $n-1$ hyperplanes to eventually obtain the hyperelliptic curve $C$ from an irreducible reduced component in the intersection. We call their reduction algorithm, together with the fastest known algorithm for solving the hyperelliptic curve discrete logarithm problem (see Subsection 4.2), the GHS attack on the ECDLP. The following theorem is proved in [17].

TheOrem I (GAUdRY, Hess AND SMART [I7]). Let $q=2^{l}$, and let

$$
E: y^{2}+x y=x^{3}+a x^{2}+b
$$

be an elliptic curve defined over $K=\mathbb{F}_{q^{n}}$. Let $\sigma: K \rightarrow K$ be the Frobenius automorphism defined by $\alpha \mapsto \alpha^{q}$, and let $b_{i}=\sigma^{i}(b)$ for $0 \leqslant i \leqslant n-1$. Let the magic number for $E$ relative to $n$ be

$$
m=m(b)=\operatorname{dim}_{\mathbb{F}_{2}}\left(\operatorname{Span}_{\mathbb{F}_{2}}\left\{\left(1, b_{0}^{1 / 2}\right),\left(1, b_{1}^{1 / 2}\right), \ldots,\left(1, b_{n-1}^{1 / 2}\right)\right\}\right) .
$$

Assume that

$$
n \text { is odd, or } \quad m(b)=n, \quad \text { or } \quad \operatorname{Tr}_{K / \mathbb{F}_{2}}(a)=0 .
$$

Then the GHS reduction constructs an explicit group homomorphism

$$
\phi: E\left(\mathbb{F}_{q^{n}}\right) \rightarrow J_{C}\left(\mathbb{F}_{q}\right),
$$

where $C$ is a hyperelliptic curve defined over $\mathbb{F}_{q}$ of genus $g$ equal to $2^{m-1}$ or $2^{m-1}-1$.

REMARK 2 (SOLVING ECDLP INSTANCES IN $E\left(\mathbb{F}_{q^{n}}\right)$ ). Assume now that \#E( $\left.\mathbb{F}_{q^{n}}\right)$ is almost prime; that is, $\# E\left(\mathbb{F}_{q^{n}}\right)=r d$, where $r$ is prime and $d$ is small. In [17] it is argued that it is highly unlikely that the kernel of $\phi$ will contain the subgroup of order $r$ of $E\left(\mathbb{F}_{q^{n}}\right)$ unless $E$ is defined over a proper subfield of $\mathbb{F}_{q^{n}}$ containing $\mathbb{F}_{q}$. Thus, $\phi$ can be used to reduce instances of the ECDLP in $\langle P\rangle$, where $P$ is a point of order $r$ in $E\left(\mathbb{F}_{q^{n}}\right)$, to instances of the HCDLP in $J_{C}\left(\mathbb{F}_{q}\right)$. In other words, given $P, Q \in\langle P\rangle$, then $\log _{P} Q=\log _{\phi(P)} \phi(Q)$. 
REMARK 3 (EFFICIENCY OF DETERMINING $C$ AND COMPUTING $\phi$ ). The running-time complexity of the algorithm presented in [17] for finding the defining equation of $C$ and for computing $\phi$ has not been determined. However, if $n g$ is relatively small, say $n g \leqslant 1000$, our extensive experiments suggest that Hess's KASH implementation [18, 6] of the algorithm takes at most a few hours on a workstation.

Formula (5) was analyzed in [23], and Theorem 5 below was obtained. We first need to define the type of an element of $\mathbb{F}_{q^{n}}$.

Definition 4. Let $n=2^{e} n_{1}$, where $n_{1}$ is odd. Let $h=2^{e}$ and $x^{n}-1=\left(f_{0} f_{1} \cdots f_{s}\right)^{h}$, where $f_{0}=x-1$ and the $f_{i}$ are distinct irreducible polynomials over $\mathbb{F}_{2}$ with $\operatorname{deg}\left(f_{i}\right)=d_{i}$ and $1=d_{0}<d_{1} \leqslant d_{2} \leqslant \cdots \leqslant d_{s}$. For $b \in \mathbb{F}_{q^{n}}$, let $\operatorname{Ord}_{b}(x)$ be the unique polynomial $f \in \mathbb{F}_{2}[x]$ of least degree such that $f(\sigma) b=0$; we have $\operatorname{Ord}_{b}(x) \mid\left(x^{n}-1\right)$. For each $i \in[0, s]$, let $j_{i}$ be the largest power of $f_{i}$ that divides $\operatorname{Ord}_{b}(x)$. The type of $b$ is defined to be $\left(j_{0}, j_{1}, \ldots, j_{s}\right)$.

Theorem 5 (see [23]). Let $b \in \mathbb{F}_{q^{n}}$ have type $\left(j_{0}, j_{1}, \ldots, j_{s}\right)$.

(i) Then $m(b)=\sum_{i=0}^{s} j_{i} d_{i}+c$, where $c=1$ if $j_{0}=0$, and $c=0$ if $j_{0} \neq 0$.

(ii) The number of elements $b \in \mathbb{F}_{q^{n}}$ of type $\left(j_{0}, j_{1}, \ldots, j_{s}\right)$ is

$$
\prod_{i=0, j_{i} \neq 0}^{s}\left(q^{j_{i} d_{i}}-q^{\left(j_{i}-1\right) d_{i}}\right) \text {. }
$$

Lemma 6 asserts that condition (6) of Theorem 1 can be weakened.

LemMa 6. Let $E / \mathbb{F}_{q^{n}}$ be an elliptic curve defined by the equation $y^{2}+x y=x^{3}+a x^{2}+b$, where $b \in \mathbb{F}_{q^{n}}$ has type $\left(j_{0}, j_{1}, \ldots, j_{s}\right)$. In Theorem 1 , condition (6) can be replaced by the following, weaker, condition:

$$
n \text { is odd, } \text { or } \quad j_{0}=2^{e}, \quad \text { or } \quad \operatorname{Tr}_{K / \mathbb{F}_{2}}(a)=0 .
$$

Proof. Observe first that if $n$ is even and $m(b)=n$, then $b$ must be of type $\left(2^{e}, \ldots, 2^{e}\right)$, so that $j_{0}=2^{e}$. Thus, (6) indeed implies (8).

Now, let $\bar{f}=(x-1)^{c} \prod_{i=0}^{s} f_{i} j_{i}$, where $c=1$ if $j_{0}=0$, and $c=0$ if $j_{0} \neq 0$. (This function has to replace the function $f$ incorrectly defined in [17, Proof of Lemma 11].) Let $\bar{h}=\left(x^{n}-1\right) / \bar{f}$. From [17, Proof of Lemma 11] it follows that Theorem 1 is true if $\operatorname{Tr}_{K / \mathbb{F}_{2}}(a)=0$ or $\operatorname{Tr}_{K / \mathbb{F}_{2}}(a)+\bar{h}(1)=0$. Thus, if $\operatorname{Tr}_{K / \mathbb{F}_{2}}(a)=1$, Theorem 1 is true if $\bar{h}(1)=1$. Since $x^{n}-1=\left(x^{n_{1}}-1\right)^{2^{e}}=(x-1)^{2^{e}} \cdot \widetilde{k}$ with $\widetilde{k}(1)=1$, we have $\bar{h}(1)=1$ if and only if $(x-1)^{2^{e}}$ divides $\bar{f}$. Since the latter is true if and only if $n$ is odd or $j_{0}=2^{e}$, the lemma is established.

The following equivalent formulation, which has been adapted from [33], is also useful.

LEMma 7. Condition (8) is equivalent to the following condition:

$$
\operatorname{gcd}\left(\frac{x^{n}-1}{\operatorname{lcm}\left(\operatorname{Ord}_{b}(x), x-1\right)}, x-1\right)=1 \quad \text { or } \quad \operatorname{Tr}_{K / \mathbb{F}_{2}}(a)=0 .
$$

Proof. Let $H(x)=\operatorname{gcd}\left(\left(x^{n}-1\right) /\left(\operatorname{lcm}\left(\operatorname{Ord}_{b}(x), x-1\right), x-1\right)\right)$. We show that $H(x)=1$ if and only if $n$ is odd or $j_{0}=2^{e}$. If $n$ is odd, then $x-1$ exactly divides $x^{n}-1$, and thus 
$H(x)=1$. Now assume that $n$ is even. Then $n=2^{e} n_{1}$ with $n_{1}$ odd and $e \geqslant 1$. Let $h=2^{e}$. Since $\operatorname{Ord}_{b}(x)$ divides $x^{n}-1$ and $x^{n}-1=\left(f_{0} f_{1} \cdots f_{s}\right)^{h}$, we have

$$
\begin{aligned}
H(x)=1 & \Longleftrightarrow(x-1) \nmid \frac{x^{n}-1}{\operatorname{lcm}\left(\operatorname{Ord}_{b}(x), x-1\right)} \\
& \Longleftrightarrow f_{0}{ }^{h} \| \operatorname{Ord}_{b}(x) \\
& \Longleftrightarrow j_{0}=h .
\end{aligned}
$$

This completes the proof.

There are $2^{N+1}-2$ isomorphism classes of elliptic curves over $\mathbb{F}_{2^{N}}$ with representatives $y^{2}+x y=x^{3}+b$ and $y^{2}+x y=x^{3}+a x^{2}+b$, where $b \in \mathbb{F}_{2^{N}}^{*}$ and $a \in \mathbb{F}_{2^{N}}$ is a fixed

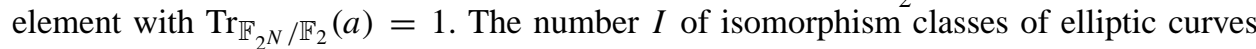
over $\mathbb{F}_{2^{N}}$ with a given magic number $m$ relative to $n$ and satisfying (8) can be efficiently computed using the following lemma.

LEMma 8. Let $n, m \in[1, n]$ be fixed. Let $c_{i, j}=q^{j d_{i}}-q^{(j-1) d_{i}}$ for $0 \leqslant i \leqslant s$ and $1 \leqslant j \leqslant h$. Let

$$
F_{0}(z)= \begin{cases}2\left(z+\sum_{j=1}^{h} c_{0, j} z^{j}\right), & \text { if } n \text { is odd } \\ z+\sum_{j=1}^{h-1} c_{0, j} z^{j}+2 c_{0, h} z^{h}, & \text { if } n \text { is even } .\end{cases}
$$

Let $F_{i}(z)=1+\sum_{j=1}^{h} c_{i, j} z^{j d_{i}}$ for $1 \leqslant i \leqslant s$, and let $F(z)=F_{0}(z) \prod_{i=1}^{s} F_{i}(z)$. Then the number of isomorphism classes of elliptic curves over $\mathbb{F}_{2^{N}}$ with magic number $m$ relative to $n$ and satisfying (8) is $I=\left[z^{m}\right] F(z)$, where '[ ]' denotes the coefficient operator.

Proof. This follows immediately from Theorem 5 and Lemma 6.

In Section 5, we shall be interested in cryptographically interesting elliptic curves that have a magic number $m$ relative to $n$. For certain pairs $(n, m)$, some (or even all) of the elliptic curves that have a magic number $m$ relative to $n$ should be eliminated from consideration because they are defined over a proper subfield $\mathbb{F}_{q^{\mu}}$ of $\mathbb{F}_{q^{n}}$ (with $q^{\mu} \geqslant 8$ ), in which case $\# E\left(\mathbb{F}_{q^{\mu}}\right)$ is a non-trivial factor of $\# E\left(\mathbb{F}_{q^{n}}\right)$, whence $E$ is not cryptographically interesting. Such subfield curves can be identified by the following result.

LEMMA 9. Let $E / \mathbb{F}_{q^{n}}$ be an elliptic curve defined by the equation $y^{2}+x y=x^{3}+a x^{2}+b$, and suppose that (9) holds. Let $\mu$ be the smallest divisor of $n$ such that $\operatorname{Ord}_{b}(x)$ divides $x^{\mu}-1$ over $\mathbb{F}_{2}$. Then $E$ is isomorphic to an elliptic curve defined over $\mathbb{F}_{q^{\mu}}$.

Proof. By assumption, we have $\sigma^{\mu}(b)-b=0$; that is, $b^{q^{\mu}}=b$. Therefore, $b \in \mathbb{F}_{q^{\mu}}$, and (because of the minimality of $\mu$ ) $b$ is not contained in a proper subfield of $\mathbb{F}_{q^{\mu}}$. Now, if $n / \mu$ is odd, there exists an element $c \in \mathbb{F}_{q^{\mu}}$ such that $\operatorname{Tr}_{K / \mathbb{F}_{2}}(c)=1$. Therefore, both for $\operatorname{Tr}_{K / \mathbb{F}_{2}}(a)=0$ and for $\operatorname{Tr}_{K / \mathbb{F}_{2}}(a)=1$, there exists a curve isomorphic to $E$ that is defined over $\mathbb{F}_{q^{\mu}}$ but not over any proper subfield of $\mathbb{F}_{q^{\mu}}$. On the other hand, if $n / \mu$ is even, then $\mu$ divides $n / 2$ and $x^{\mu}-1$ divides $x^{n / 2}-1$. Since $\operatorname{lcm}\left(\operatorname{Ord}_{b}(x), x-1\right)$ divides $x^{\mu}-1$, this implies that $\operatorname{gcd}\left(\left(x^{n}-1\right)\left(\operatorname{lcm}\left(\operatorname{Ord}_{b}(x), x-1\right)\right), x-1\right)=x-1$, so that by (9) we have $\operatorname{Tr}_{K / \mathbb{F}_{2}}(a)=0$. Again, it follows that there exists a curve isomorphic to $E$ that is defined over $\mathbb{F}_{q^{\mu}}$ but not over any proper subfield of $\mathbb{F}_{q^{\mu}}$.

Corollary io. Let $E / \mathbb{F}_{q^{n}}$ be an elliptic curve defined by the equation $y^{2}+x y=x^{3}+$ $a x^{2}+b$, and suppose that (9) holds. Then $E$ is isomorphic to an elliptic curve defined over a proper subfield $\mathbb{F}_{q^{\mu}}$ of $\mathbb{F}_{q^{n}}$ if and only if $\operatorname{Ord}_{b}(x)$ divides $x^{\mu}-1$ for some proper divisor $\mu$ of $n$. 
If $n$ is an odd prime, we have the following theorem.

THEOREM I I (see [23]). Let $n$ be an odd prime, let $\bar{t}$ be the multiplicative order of 2 modulo $n$, and let $s=(n-1) / \bar{t}$. Then the following statements hold.

(i) $x^{n}-1$ factors over $\mathbb{F}_{2}$ as $(x-1) f_{1} f_{2} \cdots f_{s}$, where the functions $f_{i}$ are distinct irreducible polynomials of degree $\bar{t}$.

(ii) The smallest admissible value of $m(b)$ greater than 1 is $m(b)=\bar{t}+1$.

(iii) Let $\sigma: \mathbb{F}_{q^{n}} \rightarrow \mathbb{F}_{q^{n}}$ be the Frobenius map defined by $x \mapsto x^{q}$. Define

$$
B=\left\{b \in \mathbb{F}_{q^{n}} \backslash \mathbb{F}_{q}:(\sigma-1) f_{i}(\sigma)(b)=0 \text { for some } 1 \leqslant i \leqslant s\right\},
$$

and let $a \in \mathbb{F}_{q^{n}}$ be an element of trace 1 . Then for all $b \in B$, the elliptic curves $y^{2}+x y=x^{3}+b$ and $y^{2}+x y=x^{3}+a x^{2}+b$ have $m(b)=\bar{t}+1$. Furthermore, no element $b \in \mathbb{F}_{q^{n}} \backslash B$ has $m(b)=\bar{t}+1$.

(iv) The cardinality of the set $B$ is $q s\left(q^{\bar{t}}-1\right)$.

\section{Algorithms for the ECDLP and HCDLP}

\section{1. $E C D L P$}

Let $E / \mathbb{F}_{2^{N}}$ be a cryptographically interesting elliptic curve, and let $r$ be the large prime divisor of $\# E\left(\mathbb{F}_{2^{N}}\right)$. Then Pollard's rho algorithm $[29,15,34]$ for solving the ECDLP in the subgroup of order $r$ of $E\left(\mathbb{F}_{2^{N}}\right)$ has an expected running time of $(\sqrt{\pi r}) / 2$ elliptic curve additions. Since $E$ is cryptographically interesting, $r \approx 2^{N-1}$ (taking into account that there is always a cofactor at least 2 ). We henceforth use $\left(\sqrt{\pi 2^{N-1}}\right) / 2$ to express the running time of Pollard's rho algorithm. Note that the algorithm can be effectively parallelized (see [26]), so that its expected running time on a network of $S$ processors is $\left(\sqrt{\pi 2^{N-1}}\right) /(2 S)$.

\section{2. $H C D L P$}

Let $C$ be a genus $g$ hyperelliptic curve over $k=\mathbb{F}_{q}$. The HCDLP is the following: given $C, D_{1} \in J_{C}(k), r=\operatorname{ord}\left(D_{1}\right)$, and $D_{2} \in\left\langle D_{1}\right\rangle$, find the integer $\lambda \in[0, r-1]$ such that $D_{2}=\lambda D_{1}$. We shall assume that $r$ is prime.

Definition I2 (ENGE-GAUdRY ALGorithm). We describe the Enge-Gaudry (EG) indexcalculus algorithm $[\mathbf{1 6}, \mathbf{8}]$ for the HCDLP. A reduced divisor $D=\operatorname{div}(a, b) \in J_{C}(k)$ is called a prime divisor if $a$ is irreducible over $k$. Each reduced divisor $D=\operatorname{div}(a, b) \in$ $J_{C}(k)$ can be expressed as a sum of prime divisors as follows: if $a=a_{1}^{e_{1}} a_{2}^{e_{2}} \cdots a_{L}^{e_{L}}$ is the factorization of $a$ into monic irreducibles over $k$, then $D=\sum_{i=1}^{L} e_{i} \operatorname{div}\left(a_{i}, b_{i}\right)$, where $b_{i}=b \bmod a_{i}$ for all $i \in[1, L]$. Such a $D$ is said to be $t$-smooth if $\max \left\{\operatorname{deg} a_{i}\right\} \leqslant t$.

In the Enge-Gaudry algorithm, a smoothness bound $t$ is first chosen. Next, the factor base $\left\{P_{1}, P_{2}, \ldots, P_{w}\right\}$ is constructed: for each prime divisor $D=\operatorname{div}(a, b)$ of degree less than or equal to $t$, exactly one of $D$ and $-D$ is included in the factor base. Then, a random walk (á la Teske [32]) is performed in the set of reduced divisors equivalent to divisors of the form $\alpha D_{1}+\beta D_{2}$ and the $t$-smooth divisors encountered in this walk are stored; each $t$-smooth divisor yields a relation $\alpha_{i} D_{1}+\beta_{i} D_{2} \sim R_{i}=\sum_{j} e_{i j} P_{j}$. When $w+5$ different relations have been found, one can find by linear algebra modulo $r$ a non-trivial linear combination

$$
\sum_{i=1}^{w+5} \gamma_{i}\left(e_{i 1}, e_{i 2}, \ldots, e_{i w}\right)=(0,0, \ldots, 0) \text {. }
$$


Thus $\sum_{i=1}^{w+5} \gamma_{i} R_{i}=0$, whence

$$
\sum \gamma_{i}\left(\alpha_{i} D_{1}+\beta_{i} D_{2}\right)=0 \text { and } \log _{D_{1}} D_{2}=-\left(\sum \gamma_{i} \alpha_{i}\right) /\left(\sum \gamma_{i} \beta_{i}\right) \bmod r \text {. }
$$

\subsection{Analysis}

The EG algorithm has a subexponential-time running time of

$$
O\left(\exp \left((\sqrt{2}+o(1)) \sqrt{\log q^{g} \log \log q^{g}}\right)\right)
$$

bit operations for $g / \log q \rightarrow \infty$. The following non-asymptotic analysis of the running time for the relation-gathering stage was given in [20]. A good approximation for the number $A_{l}$ of prime divisors of degree $l$ in the factor base is

$$
A_{l} \approx \frac{1}{2}\left(\frac{1}{l} \sum_{d \mid l} \mu\left(\frac{l}{d}\right) q^{d}\right),
$$

where $\mu$ is the Möbius function. The factor base size $w$ is therefore well approximated by

$$
F(t)=\sum_{l=1}^{t} A_{l}=\frac{1}{2} \sum_{l=1}^{t}\left(\frac{1}{l} \sum_{d \mid l} \mu\left(\frac{l}{d}\right) q^{d}\right) .
$$

By [20, Lemma 2], the number of $t$-smooth reduced divisors in $J_{C}(k)$ is

$$
M(t)=\sum_{i=1}^{g}\left(\left[x^{i}\right] \prod_{l=1}^{t}\left(\frac{1+x^{l}}{1-x^{l}}\right)^{A_{l}}\right),
$$

where ' $[$ ]' denotes the coefficient operator. Under the heuristic assumption that the proportion of $t$-smooth divisors in $\left\langle D_{1}\right\rangle$ is the same as the proportion of $t$-smooth divisors in the full group $J_{C}(k)$, the expected number of random-walk iterations before a $t$-smooth divisor is encountered is

$$
E(t)=\# J_{C}(k) / M(t) .
$$

Finally, the expected number of random-walk iterations before $F(t)+5$ relations are generated is

$$
T(t)=(F(t)+5) E(t)
$$

The system of sparse linear equations can be solved using Lanczos's algorithm [5]. A good estimate for the expected running time is $\alpha(F(t)+5)^{2}$ arithmetic operations modulo $n$, where $\alpha$ is the average number of non-zero coefficients in an equation. Since $\alpha \leqslant g$, the approximation

$$
L(t)=F(t)^{2}
$$

that we will use henceforth for the running time of the linear algebra stage is a reasonably good one.

\section{Analysis}

For each composite $N \in[100,600]$, we determine and compare the running times of the GHS attack and Pollard's rho method for solving the ECDLP in (potentially) cryptographically interesting elliptic curves over $\mathbb{F}_{2^{N}}$. 
Algorithm 13 determines the elliptic curve parameters (in terms of $n, m$ and $g$ ) such that:

(i) there should exist (see Remark 21) a cryptographically interesting elliptic curve $E$ over $\mathbb{F}_{2^{N}}$ with these parameters; and

(ii) the GHS attack is more efficient for solving the ECDLP in $E\left(\mathbb{F}_{2^{N}}\right)$ than for solving the ECDLP on any other cryptographically interesting elliptic curve over $\mathbb{F}_{2^{N}}$.

\subsection{Cases of Algorithm 13}

Three cases are considered.

Case 1. The first case, denoted by EG1, considers only GHS attack parameters where the factor base has size at most $10^{7} \approx 2^{23}$. Solving sparse linear systems of this dimension is on the edge of what is considered feasible today [21]. Thus the EG1 running times are restricted to ECDLP instances where the linear algebra stage of the GHS attack is feasible today.

If the number $A_{1}$ of degree-one divisors is greater than $10^{7}$ for some hyperelliptic curve of genus $g$ over $\mathbb{F}_{2^{l}}$, then, in order to achieve a factor base size less than or equal to $10^{7}$, the Enge-Gaudry algorithm could be modified by selecting the factor base to consist of only a proportion $1 / \varepsilon$ of all prime divisors of degree 1; see [17]. However, the expected time to find a smooth divisor will be increased by a factor of $\varepsilon^{g}$. We therefore decided not to consider this modification in our analysis.

Case 2. The second case, denoted by EG2, places no restriction on the factor base size, nor does it take into account the running time of the linear algebra stage when selecting the optimal elliptic curve parameters. Listing EG2 running times is important, because they will become relevant should faster algorithms be discovered for solving sparse linear systems.

Case 3. The third case, denoted by EG3, places no restriction on the factor base size, but does consider the running time of the linear algebra stage when selecting the optimal elliptic curve parameters.

\subsection{Running times}

We express the running time for Pollard's rho method in terms of elliptic curve operations. For the EG1 and EG2 cases, the running times for the GHS attack are expressed in terms of random-walk iterations in the Jacobian of the hyperelliptic curve. We do not consider the different bit-complexities of operations for elliptic and hyperelliptic curves, since these are expected to be roughly the same. For the running time of the GHS attack in the EG3 case, both the number of random-walk iterations and the number of steps in the linear algebra stage are considered. Comparing the running times of the two stages is problematic because of the different bit-complexities for the basic operations involved and because, unlike the linear algebra stage, the random-walk stage can be easily parallelized on a large distributed system of computers, since the individual processors do not have to communicate with each other. Nevertheless, for the sake of concreteness, we select GHS attack parameters in the EG3 case, so that the maximum of the running times of the two stages is minimized. As was justified in Remark 3, we can ignore the time spent on mapping the ECDLP instance to a HCDLP instance in each of the EG1, EG2 and EG3 cases. 


\section{AlgORITHM I3 (FINDING OPTIMAL GHS ATTACK PARAMETERS).}

InPut: $N$, 'EG1' or 'EG2' or 'EG3'.

OUTPUT: Parameters $n, m$ and $g$, for which there should exist a cryptographically interesting elliptic curve over $\mathbb{F}_{2^{N}}$ whose ECDLP is most easily solved with the GHS attack; optimal smoothness bound $t$; (estimated) factor base size $F$; (estimated) expected running time $T$ in terms of random-walk iterations; and, in the EG3 case, (estimated) maximum $T_{M}$ of the running times of the random-walk and linear algebra stages.

1. For all divisors $n \geqslant 2$ of $N$, do the following.

(a) Set $l \leftarrow N / n$ and $q \leftarrow 2^{l}$.

(b) $\left\{\right.$ For EG1: The $10^{7}$ bound on the factor base size must be violated if $A_{1}=$ $2^{l-1}>10^{7}$. $\}$

Case EG1: If $l \geqslant 25$, then set $T_{n} \leftarrow \infty$ and go to step 1 .

(c) Write $n=n_{1} h$ where $h=2^{e}$ and $n_{1}$ is odd.

(d) $\left\{\right.$ Find the irreducible factors of $x^{n_{1}}-1$ over $\mathbb{F}_{2}$ and their degrees.

Factor $x^{n_{1}}-1=f_{0}(x) f_{1}(x) \cdots f_{s}(x)$, where the $f_{i}$ are irreducible over $\mathbb{F}_{2}$, and where $1=d_{0} \leqslant d_{1} \leqslant d_{2} \leqslant \cdots \leqslant d_{s}$, where $d_{i}=\operatorname{deg}\left(f_{i}\right)$.

(e) \{ Compute a lower bound $m^{\prime}$ on the magic number $m$ relative to $n$ that yields a large enough Jacobian (see Remark 14). \}

For $m^{\prime}=2,3, \ldots, n$, do the following.

(i) Set $g \leftarrow 2^{m^{\prime}-1}-1$. Compute $B_{1}$ and $B_{2}$ as defined in (4) and (3).

(ii) If $\min \left\{\log _{2} B_{1}, \log _{2} B_{2}\right\} \geqslant N-3$, then go to step 1(f).

(iii) Set $g \leftarrow 2^{m^{\prime}-1}$. Compute $B_{1}$ and $B_{2}$ as defined in (4) and (3).

(iv) If $\min \left\{\log _{2} B_{1}, \log _{2} B_{2}\right\} \geqslant N-3$, then go to step 1 (f).

(f) $\{$ Find the smallest admissible magic number $m$ relative to $n$ (see Theorem 5). $\}$ For $m=m^{\prime}, m^{\prime}+1, \ldots, n$, do the following.

If $m$ can be written in the form $\sum_{i=0}^{s} d_{i} j_{i}$ with $0 \leqslant j_{i} \leqslant h, j_{0} \geqslant 1$, then:

$\{$ Determine if there are any elliptic curves having magic number $m$ relative to $n$ that are not defined over a proper subfield $\mathbb{F}_{q^{\mu}}$ of $\mathbb{F}_{q^{n}}$ (see Remark 15). \}

For each expression $m=\sum_{i=0}^{s} d_{i} j_{i}$ with $0 \leqslant j_{i} \leqslant h, j_{0} \geqslant 1$, do the following.

(i) Let $f(x)=\prod_{i=0}^{s} f_{i}(x)^{j_{i}}$.

(ii) Let $\mu$ be the smallest divisor of $n$ such that $f(x)$ divides $x^{\mu}-1$.

(iii) If $\mu=n$, then go to step $1(\mathrm{~g})$.

(g) If $m>m^{\prime}$, then set $g \leftarrow 2^{m-1}-1$.

(h) $\{$ If the size of the Jacobian is not too large (that is, if $g l \leqslant 4096$; see Remark 16), then find the optimum smoothness bound $t$ for the Enge-Gaudry algorithm using:

(11) to estimate the factor base size $F(t)$,

(13) to estimate the expected running time $E(t)$ to find a smooth divisor with $\# J_{C}\left(\mathbb{F}_{q}\right)=2^{g l}$,

(14) to estimate the expected running time $T(t)$ of the random-walk stage, and

(15) to estimate the running time $L(t)$ of the linear algebra stage. $\}$

If $g l \geqslant 4097$, then set $T_{n} \leftarrow \infty$.

Else:

(i) Case EG1: set $S \leftarrow\left\{1 \leqslant t \leqslant 120: F(t) \leqslant 10^{7}\right\}$.

Case EG2 or EG3: set $S \leftarrow\{1,2, \ldots, 120\}$. 
(ii) Case EG1 or EG2: let $t$ be the index in $S$ that minimizes $T(t)$. Case EG3: let $t$ be the index in $S$ that minimizes $T_{M}(t)=\max \{T(t), L(t)\}$.

(iii) Set $m_{n} \leftarrow m ; g_{n} \leftarrow g$; $t_{n} \leftarrow t ; F_{n} \leftarrow F\left(t_{n}\right)$.

Case EG1 or EG2: set $T_{n} \leftarrow T\left(t_{n}\right)$.

Case EG3: set $T_{n} \leftarrow T_{M}\left(t_{n}\right)$.

2. If $T_{n}=\infty$ for all $n$, output ' $g l \geqslant 4097$ for all $n$ '.

Else, let $n$ be the index for which $T_{n}$ is a minimum, and output ' $\left(n, m_{n}, g_{n}, t_{n}, F_{n}, T_{n}\right)$ '.

\subsection{Explanations of some steps of Algorithm 13}

REMARK I4 (LOWER BOUND ON $\log _{2} B_{1}$ AND $\log _{2} B_{2}$ ). If we restrict our attention to cryptographically interesting elliptic curves $E$ over $\mathbb{F}_{2^{N}}$ with $\# E\left(\mathbb{F}_{2^{N}}\right)=d r$, where $d \in\{2,4\}$ and $r$ is prime, then

$$
r \geqslant \# E\left(\mathbb{F}_{2^{N}}\right) / 4 \geqslant\left(2^{N / 2}-1\right)^{2} / 4>2^{N-1} / 4=2^{N-3}, \quad \text { for } N \geqslant 4 .
$$

Thus, if the hyperelliptic curve $C$ over $\mathbb{F}_{q}$ generated by the GHS reduction has genus $g$, then by (3) and (4) a necessary condition for $J_{C}\left(\mathbb{F}_{q}\right)$ to have a subgroup of order $r$ is $\min \left(B_{1}, B_{2}\right) \geqslant \# J_{C}\left(\mathbb{F}_{q}\right) \geqslant 2^{N-3}$.

REMARK I5 (ELIMINATION OF SUBFIELD CURVES). There are some $(N, l, g)$ parameters for which elliptic curves over $\mathbb{F}_{2^{N}}$ with parameters $(l, g)$ do exist, but none of these is cryptographically interesting. For example, if $N=160$, the ECDLP is most easily solved with the GHS attack if $(n, l, m, g)=(8,20,4,8)$. Then, for the attack to work (see Lemma 6 ,

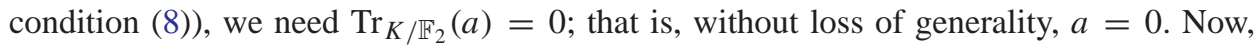
consider an elliptic curve $E: y^{2}+x y=x^{3}+b$ over $\mathbb{F}_{2}{ }^{160}$ that yields the magic number $m=4$ on performing the GHS attack with $n=8$. We have $x^{n}-1=(x-1)^{8}$, and hence $(\sigma-1)^{4} b=0$ where $\sigma: \mathbb{F}_{2^{160}} \rightarrow \mathbb{F}_{2^{160}}$ is defined by $\alpha \mapsto \alpha^{2^{20}}$. That is, $b \in \mathbb{F}_{2^{80}}$, which implies that $\# E\left(\mathbb{F}_{2^{80}}\right)$ divides $\# E\left(\mathbb{F}_{2}{ }^{160}\right)$. Hence $E$ is not cryptographically interesting. The next easiest instance of an ECDLP over $\mathbb{F}_{2160}$ for which a cryptographically interesting curve can exist is $(n, l, m, g)=(20,8,6,31)$. Such a phenomenon always occurs when $(n, m)=(8,4)$ are the GHS parameters for which the ECDLP is most easily solved, which is the case for $N=176,184,192$ and many other $N$ divisible by 8 . But also for $N=224$, where $(n, m)=(32,6)$ would be best, we find that $\# E\left(\mathbb{F}_{256}\right)$ must divide $\# E\left(\mathbb{F}_{2224}\right)$ for any elliptic curve with these parameters. Another example is $N=304$, where $(n, m)=(16,5)$ would be optimal; here we find that $\# E\left(\mathbb{F}_{2^{304}}\right)$ must be divisible by $\# E\left(\mathbb{F}_{2^{152}}\right)$. Corollary 10 is therefore used in step 1(f) of Algorithm 13 to eliminate from consideration elliptic curves defined over a proper subfield $\mathbb{F}_{q^{\mu}}$ of $\mathbb{F}_{q^{n}}$. Note that step 1(f) does not exclude elliptic curves $E$ defined over proper subfields of $\mathbb{F}_{q^{n}}$ not containing $\mathbb{F}_{q}$. However, we expect that such elliptic curves will be uniformly distributed among the classes of curves identified by $\operatorname{Ord}_{b}(x)$ (relative to the Frobenius map $\alpha \mapsto \alpha^{q}$ ), so they will not significantly affect the counts $I$ of potentially cryptographically interesting elliptic curves having a certain magic number $m$ relative to $n$.

REMARK I 6 (THE RESTRICTION THAT $g l \leqslant 4096$ ). For $g \geqslant 4097$, we were unable to compute the expected running time of EG1, EG2 or EG3 because of computational limitations when computing the Taylor series expansions needed to evaluate $M(t)$ (see formula (12)). We therefore ignore all instances $(n, l, g)$ where $g l \geqslant 4097$. Notice that in this case the 
Jacobian $J_{C}\left(\mathbb{F}_{q}\right)$ has size at least $2^{4097}$, whence any (cryptographically interesting) HCDLP instance in $J_{C}\left(\mathbb{F}_{q}\right)$ is infeasible using the known index-calculus algorithms. In particular, if $l=1$ and $g=4095$, the smallest running time for EG2 is obtained with $t=120$, and amounts to $\approx 2^{307}$ random-walk iterations, which is more than the expected number of elliptic curve operations using Pollard's rho method for $N=600$.

\subsection{Analysis}

The outputs of Algorithm 13 with composite $N \in[100,600]$ as inputs are listed in Appendix A. For the purposes of illustration, a small excerpt from this table is given in Table 1. In these tables, the entries for $F, T, T_{M}$ and $\rho$ are the logarithms (base 2, rounded to the nearest integer) of the factor base size $F(t)$, the expected number $T(t)$ of randomwalk iterations in the Enge-Gaudry algorithm, the maximum $T_{M}(t)$ of $T(t)$ and $L(t)$, and the number of elliptic curve operations in Pollard's rho method, respectively. $D 1$ and $D 2$ denote the differences $\rho-T$ (if positive) for EG1 and EG2, respectively, while $D 3$ denotes the difference $\rho-T_{M}$ (if positive) for EG3. $I$ denotes the logarithm (base 2, rounded) of the number of isomorphism classes of elliptic curves which have a magic number $m$ relative to $n$ satisfying (8), and which are not defined over a proper subfield $\mathbb{F}_{q^{\mu}}$ of $\mathbb{F}_{q^{n}}$. If for some $N$ data is given for EG2 or EG3 but not for EG1, we are in the situation that $g l \geqslant 4097$ for all divisors $l \leqslant 24$ of $N$ (such as for $N=164$ and 166 in Table 1). If for some $N$ data is given for none of EG1, EG2 or EG3, we are in the situation that $g l \geqslant 4097$ for all $l$ dividing $N$ (such as for $N=169$ in Table 1).

REMARK I7 (FURTHER LIMITATIONS OF OUR ANALYSIS). Our analysis yields the same running times whenever $(g, l)$ are the same, independently of $N$ (for example, $T=59$ when $(g, l)=(15,20)$ for both $N=160$ and $N=280$ in the EG1 case; see Appendix A). This is because the running time of the Enge-Gaudry algorithm is computed under the assumption that $\# J_{C}\left(\mathbb{F}_{q}\right) \approx q^{g}=2^{g l}$. However, we expect that $\# J_{C}\left(\mathbb{F}_{q}\right)$ will be divisible only by the large prime that divides $\# E\left(\mathbb{F}_{q^{n}}\right)$. Hence if $g l \gg N$, it may well be the case that the Jacobian obtained from Weil descent is much smaller in size than $q^{g}$, which would then lead to a significantly smaller value $E(t)=\# J_{C}(k) / M(t)$, and hence also to a significantly smaller running time $T(t)$. This observation is particularly meaningful where $l=1$, in which case the Hasse-Weil lower bound $(\sqrt{2}-1)^{2 g} \leqslant \# J_{C}\left(\mathbb{F}_{2}\right)$ is trivial. For example, if $(l, g)=(1,511)$, we have $T=2^{105}$ for EG1 for $N=170,465,508,510$ and 511 . Thus, caution must be exercised when interpreting our data for those $N$ where $g l \gg N$. Nevertheless, if $g l \approx N$, our running-time estimates are precise.

REMARK I 8 (SUCCESS OF THE GHS ATTACK). There are some composite $N \in[160,600]$ for which the GHS attack succeeds on some cryptographically interesting elliptic curves over $\mathbb{F}_{2^{N}}$. That is, Pollard's rho algorithm is infeasible for solving the ECDLP on such curves, and the GHS attack is successful in reducing instances of the ECDLP on these curves to instances of the HCDLP that are solvable using known algorithms and existing computer technology. Examples of such $N$ are 161, 180, 186, 217, 248, 300 (see Section 7).

For many other composite $N \in[160,600]$, the GHS attack, though infeasible today, is successful in that it is significantly faster than Pollard's rho algorithm for solving the ECDLP on a large class of elliptic curves over $\mathbb{F}_{2^{N}}$. A striking example is $N=600$, where the GHS attack can solve the ECDLP on $2^{202}$ curves over $\mathbb{F}_{2^{600}}$ in about $2^{79}$ steps, which is less than the $2^{299}$ steps for Pollard's rho algorithm. 
Table 1: Sample output of Algorithm 13 (see Appendix A).

\begin{tabular}{cccccccccccc}
\hline EG1 & $N$ & $n$ & $l$ & $m$ & $g$ & $I$ & $t$ & $F$ & $T$ & $\rho$ & $D 1$ \\
\hline & 160 & 8 & 20 & 5 & 15 & 100 & 1 & 19 & 59 & 79 & 20 \\
& 161 & 7 & 23 & 4 & 7 & 94 & 1 & 22 & 34 & 80 & 46 \\
& 162 & 9 & 18 & 7 & 63 & 127 & 1 & 17 & 307 & 80 & - \\
& 164 & - & - & - & - & - & - & - & - & - & - \\
& 165 & 15 & 11 & 5 & 15 & 57 & 2 & 20 & 37 & 82 & 45 \\
& 166 & - & - & - & - & - & - & - & - & - & - \\
& 168 & 7 & 24 & 4 & 7 & 98 & 1 & 23 & 35 & 83 & 48 \\
& 169 & - & - & - & - & - & - & - & - & - & - \\
& 170 & 170 & 1 & 10 & 511 & 13 & 28 & 23 & 105 & 84 & -
\end{tabular}

\begin{tabular}{rrrrrrrrrrrr}
\hline EG2 & $N$ & $n$ & $l$ & $m$ & $g$ & $I$ & $t$ & $F$ & $T$ & $\rho$ & $D 2$ \\
\hline & 160 & 4 & 40 & 3 & 4 & 120 & 1 & 39 & 44 & 79 & 35 \\
& 161 & 7 & 23 & 4 & 7 & 94 & 1 & 22 & 34 & 80 & 46 \\
& 162 & 6 & 27 & 4 & 7 & 109 & 1 & 26 & 38 & 80 & 42 \\
& 164 & 4 & 41 & 3 & 4 & 123 & 1 & 40 & 45 & 81 & 36 \\
& 165 & 15 & 11 & 5 & 15 & 57 & 2 & 20 & 37 & 82 & 45 \\
& 166 & 2 & 83 & 2 & 2 & 167 & 1 & 82 & 83 & 82 & - \\
& 168 & 7 & 24 & 4 & 7 & 98 & 1 & 23 & 35 & 83 & 48 \\
& 169 & - & - & - & - & - & - & - & - & - & - \\
170 & 5 & 34 & 5 & 15 & 171 & 1 & 33 & 73 & 84 & 11
\end{tabular}

\begin{tabular}{rrrrrrrrrrrrr}
\hline EG3 & $N$ & $n$ & $l$ & $m$ & $g$ & $I$ & $t$ & $F$ & $T$ & $T_{M}$ & $\rho$ & $D 3$ \\
\hline & 160 & 8 & 20 & 5 & 15 & 100 & 1 & 19 & 59 & 59 & 79 & 20 \\
& 161 & 7 & 23 & 4 & 7 & 94 & 1 & 22 & 34 & 44 & 80 & 36 \\
& 162 & 6 & 27 & 4 & 7 & 109 & 1 & 26 & 38 & 52 & 80 & 28 \\
& 164 & 4 & 41 & 3 & 4 & 123 & 1 & 40 & 45 & 80 & 81 & 1 \\
& 165 & 15 & 11 & 5 & 15 & 57 & 2 & 20 & 37 & 40 & 82 & 42 \\
& 166 & 2 & 83 & 2 & 2 & 167 & 1 & 82 & 83 & 164 & 82 & - \\
& 168 & 28 & 6 & 6 & 31 & 37 & 4 & 21 & 41 & 42 & 83 & 41 \\
& 169 & - & - & - & - & - & - & - & - & - & - & - \\
& 170 & 5 & 34 & 5 & 15 & 171 & 1 & 33 & 73 & 73 & 84 & 11 \\
\hline
\end{tabular}

There are also many composite $N$ for which the GHS attack is infeasible today, yet it takes less time than Pollard's rho algorithm for solving the ECDLP on essentially all elliptic curves over $\mathbb{F}_{2^{N}}$. Examples of such $N$ are 170, 185, 190, 215, 220 (all with GHS attack parameters $n=5, m=5$ ). For $N=185$, the GHS attack takes about $2^{76}$ steps, versus about $2^{92}$ for Pollard's rho algorithm. This case is of practical significance because a specific elliptic curve over $\mathbb{F}_{2185}$ is listed in the IETF standard [19] for key establishment.

REMARK I9 (FAILURE OF THE GHS ATTACK). We can conclude that for those composite $N \in[100,600]$ for which $D 3<0$ for EG3, the GHS attack does not reduce the level of security offered: Pollard's rho method is the faster algorithm for all elliptic curves over $\mathbb{F}_{2^{N}}$. We emphasize that our statements about the failure of the GHS attack are made under the assumption that the Enge-Gaudry algorithm is essentially the best index-calculus algorithm for the HCDLP. 
REMARK 20 (EFFECTIVENESS OF THE GHS ATtACK). When $D 1>0$ or $D 2>0$ or $D 3>0$ for some composite $N \in[100,600]$, the level of security offered by some cryptographically interesting elliptic curves defined over $\mathbb{F}_{2^{N}}$ may be reduced by means of the GHS attack. However, note that our data corresponds to elliptic curves with least possible magic numbers and genera, and usually only a small proportion of elliptic curves yield this minimal magic number. For example, if $N=161$, then only $\approx 2^{94}$ out of $\approx 2^{162}$ elliptic curves over $\mathbb{F}_{2^{161}}$ have magic number $m=4$ relative to $n=7$. Correspondingly, for $N=165$ the proportion of non-subfield elliptic curves with magic number $m=5$ relative to $n=15$ is only $\approx 2^{57}$ out of $2^{166}$. Galbraith, Hess and Smart [14] (see also [12]) presented an algorithm with expected average running time of $O\left(q^{n / 4+\varepsilon}\right)$ for explicitly computing an isogeny between two isogenous elliptic curve over $\mathbb{F}_{q^{n}}$. (Two elliptic curves $E_{1} / \mathbb{F}_{q^{n}}$ and $E_{2} / \mathbb{F}_{q^{n}}$ are said to be isogenous over $\mathbb{F}_{q^{n}}$ if $\# E_{1}\left(\mathbb{F}_{q^{n}}\right)=\# E_{2}\left(\mathbb{F}_{q^{n}}\right)$.) They observed that this algorithm can be used to extend the effectiveness of the GHS attack. In other words, given an ECDLP instance on some cryptographically interesting elliptic curve $E_{1} / \mathbb{F}_{2^{N}}$, one can check whether $E_{1}$ is isogenous to some elliptic curve $E_{2} / \mathbb{F}_{2^{N}}$ that yields an easier HCDLP than $E_{1}$, and then use an isogeny $\phi: E_{1} \rightarrow E_{2}$ to map the ECDLP instance to an instance of the ECDLP in $E_{2}\left(\mathbb{F}_{2^{N}}\right)$. For example, in the case $N=165$, we can expect that roughly $2^{135}$ out of $2^{166}$ elliptic curves over $\mathbb{F}_{2^{165}}$ will be isogenous to one of the $\approx 2^{57}$ elliptic curves over $\mathbb{F}_{2^{165}}$ having magic number $m=5$ relative to $n=15$. Note, however, that finding a curve with $m=5$ isogenous to a given elliptic curve over $\mathbb{F}_{2^{165}}$ (assuming that such an isogenous curve exists) may be difficult, as one has essentially to search through the entire set of $2^{57}$ curves.

REMARK 2I. (FINDING CRYPTOGRAPHICALLY INTERESTING ELLIPTIC CURVES WITH GIVEN $(N, l, m)$ PARAMETERS). One can attempt to find a cryptographically interesting elliptic curve with given $(N, l, m)$ parameters as follows. First, select an arbitrary $b$ from the set

$$
B=\left\{b \in \mathbb{F}_{q^{n}}: m(b)=m \text { and } b \notin \mathbb{F}_{q^{\mu}} \text { for all proper divisors } \mu \text { of } n\right\} ;
$$

it can be seen from Theorem 5(i) that the elements of $B$ can be efficiently enumerated. Next, compute $H=\# E_{b}\left(\mathbb{F}_{2^{N}}\right)$, where $E_{b}: y^{2}+x y=x^{3}+b$, using Satoh's algorithm [30, 9], and test whether either $H$ or $2^{N+1}+2-H$ (the order of the twist of $E_{b}$ ) is almost a prime. Observe that if $b \in B$, then $b^{2} \in B$. Moreover, $E_{b}$ and $E_{b^{2}}$ are isogenous over $\mathbb{F}_{2^{N}}$. Thus, if $b \in B$ has already been tested, then one should not select $b^{2^{i}}$ for any $1 \leqslant i \leqslant N-1$. Now, it is known that the order of a randomly selected elliptic curve over $\mathbb{F}_{2^{N}}$ is roughly uniformly distributed over the even integers in the Hasse interval $\left[\left(2^{N / 2}-1\right)^{2},\left(2^{N / 2}+1\right)^{2}\right]$. Thus, if the set $B$ has sufficiently large cardinality (which can be determined from Theorem 5), then we can expect to quickly find an elliptic curve of almost prime order.

\section{Elliptic curves from ANSI X9.62}

The ANSI X9.62 standard [1, Appendix H.4] lists specific elliptic curves over fields of characteristic two of the composite extension degrees $N=176,208,272,304,368$. These $N$ factor as $16 \cdot p$, where $p \in\{11,13,17,19,23\}$ is prime. Table 2 lists the elliptic curve parameters in hexadecimal notation, where each curve is defined by the equation $y^{2}+x y=x^{3}+a x^{2}+b$. Notice that in all cases the coefficients $a$ and $b$ lie in the proper subfield $\mathbb{F}_{2^{16}}$ of $\mathbb{F}_{2^{N}}$, whence\# $E\left(\mathbb{F}_{2^{N}}\right)=r d$ with $r$ prime and $d \in\left[2^{16}+1-2^{9}, 2^{16}+1+2^{9}\right]$.

For a curve defined over a proper subfield of $\mathbb{F}_{q^{n}}$ containing $\mathbb{F}_{q}$, it cannot be argued that the kernel of the map $\phi$ defined in (7) would not contain the large subgroup of order $r$ of $E\left(\mathbb{F}_{q^{n}}\right)$. In fact, the opposite is true. 
Table 2: Sample elliptic curves from ANSI X9.62.

$\mathrm{E} 176, N=176, \mathbb{F}_{2176}=\mathbb{F}_{2}[z] /\left(z^{176}+z^{43}+z^{2}+z+1\right), \# \mathrm{E} 176\left(\mathbb{F}_{2176}\right)=65390 \cdot r$
$a=$ E4E6DB2995065C407D9D39B8D0967B96704BA8E9C90B
$b=$ 5DDA470ABE6414DE8EC133AE28E9BBD7FCEC0AE0FFF2
$r=10092537397$ ECA4F6145799D62B0A19CE06FE26AD

$\mathrm{E} 208, N=208, \mathbb{F}_{2^{208}}=\mathbb{F}_{2}[z] /\left(z^{208}+z^{83}+z^{2}+z+1\right), \# \mathrm{E} 208\left(\mathbb{F}_{2208}\right)=65096 \cdot r$ $a=0$

$b=\mathrm{C} 8619 \mathrm{ED} 45 \mathrm{~A} 62 \mathrm{E} 6212 \mathrm{E} 1160349 \mathrm{E} 2 \mathrm{BFA} 844439 \mathrm{FAFC} 2 \mathrm{~A} 3 \mathrm{FD} 1638 \mathrm{~F} 9 \mathrm{E}$ $r=101 \mathrm{BAF} 95 \mathrm{C} 9723 \mathrm{C57B6C21DA2EFF2D5ED588BDD5717E212F9D}$

$\mathrm{E} 272, N=272, \mathbb{F}_{2272}=\mathbb{F}_{2}[z] /\left(z^{272}+z^{56}+z^{3}+z+1\right), \# \mathrm{E} 272\left(\mathbb{F}_{2272}\right)=65286 \cdot r$ $a=91 \mathrm{~A} 091 \mathrm{~F} 03 \mathrm{~B} 5 \mathrm{FBA} 4 \mathrm{AB} 2 \mathrm{CCF} 4$ 9C4EDD220FB028712D42BE752B2C40094DBAC DB586FB20

$b=7167 \mathrm{EFC} 92 \mathrm{BB} 2 \mathrm{E} 3 \mathrm{CE} 7 \mathrm{C} 8 \mathrm{AAAFF} 34 \mathrm{E} 12 \mathrm{~A} 9 \mathrm{C} 557003 \mathrm{D} 7 \mathrm{C} 73 \mathrm{~A} 6 \mathrm{FAF} 003 \mathrm{~F} 99 \mathrm{~F} 6 \mathrm{CC} 8$ $482 \mathrm{E} 540 \mathrm{~F} 7$

$r=100 \mathrm{FAF} 51354 \mathrm{E} 0 \mathrm{E} 39 \mathrm{E} 48$ 92DF6E319C72C8161603FA45AA7B998A167B8F1E 629521

$\mathrm{E} 304, N=304, \mathbb{F}_{2^{304}}=\mathbb{F}_{2}[z] /\left(z^{304}+z^{11}+z^{2}+z+1\right), \# \mathrm{E} 304\left(\mathbb{F}_{2^{304}}\right)=65070 \cdot r$ $a=\mathrm{FD} 0 \mathrm{D} 693149 \mathrm{~A} 118 \mathrm{~F} 651 \mathrm{E} 6 \mathrm{DCE} 6802085377 \mathrm{E} 5 \mathrm{~F} 882 \mathrm{D} 1 \mathrm{~B} 510 \mathrm{~B} 44160074 \mathrm{C} 1288$ $078365 \mathrm{~A} 0396 \mathrm{C} 8 \mathrm{E} 681$

$b=$ BDDB97E555A50A908E43B01C798EA5DAA6788F1EA2 794EFCF57166B8C14 $039601 \mathrm{E} 55827340 \mathrm{BE}$

$r=101 \mathrm{D} 556572 \mathrm{AABAC} 800101 \mathrm{D} 556572 \mathrm{AABAC} 8001022 \mathrm{D}$ C91DD173F 8FB561DA $6899164443051 \mathrm{D}$

$\mathrm{E} 368, N=368, \mathbb{F}_{2^{368}}=\mathbb{F}_{2}[z] /\left(z^{368}+z^{85}+z^{2}+z+1\right), \# \mathrm{E} 368\left(\mathbb{F}_{2} 368\right)=65392 \cdot r$ $a=\mathrm{E} 0 \mathrm{D} 2 \mathrm{EE} 25095206 \mathrm{~F} 5 \mathrm{E} 2 \mathrm{~A} 4 \mathrm{~F}$ 9ED22 9F1F256E79A0E2B455970D8D0D865BD 44 778C576D 62F0AB7519CCD2A1A906AE30D

$b=$ FC1217D4320A90452C760A58EDCD30C8DD069B3C34453837A34ED50CB54 917E1C2112D84D164F444F8F74786046A

$r=10090512 \mathrm{DA} 9 \mathrm{AF} 72 \mathrm{~B} 0834$ 9D 98A5DD4C7B0532ECA51CE03E2D10F3B7AC579 BD87E909AE40A6F131E9CFCE5BD967 
REMARK 22 (FAILURE OF GHS-ATTACK FOR SUbFIELD CURVES). Let $E / \mathbb{F}_{q^{n}}$ be an elliptic curve defined by the equation $y^{2}+x y=x^{3}+a x^{2}+b$. Let $\mathbb{F}_{q}(a, b)$ be the smallest extension of $\mathbb{F}_{q}$ over which $E$ is defined. Then for any extension field $K$ of $\mathbb{F}_{q}(a, b)$, the GHS Weil descent of $E / K$ down to $\mathbb{F}_{q}$ is independent of $K$. That is, the GHS Weil descent of $E / K$ down to $\mathbb{F}_{q}$ yields the same (up to birational equivalence) hyperelliptic curve $C / \mathbb{F}_{q}$ as the GHS Weil descent of $E / \mathbb{F}_{q}(a, b)$. This can be derived from the facts that the defining equations for $\mathfrak{D}$ in $\left[17\right.$, Lemma 2] depend only on $\mathbb{F}_{q}(a, b)$ but not on $K$, and that the same is true for the set $\Delta_{0}$ in [17, proof of Lemma 6]. Thus, if $\mathbb{F}_{q}(a, b) \neq \mathbb{F}_{q^{n}}$, only points in the small subgroup $E\left(\mathbb{F}_{q}(a, b)\right)$ of $E\left(\mathbb{F}_{q^{n}}\right)$ are likely to be mapped to non-trivial divisors in the Jacobian $J_{C}\left(\mathbb{F}_{q}\right)$, while points in the subgroup of order $r$ will be mapped to the zero divisor, which is of no use for solving ECDLPs in $E\left(\mathbb{F}_{q^{n}}\right)$.

REMARK 23 (SUCCESS OF THE GHS-ATTACK FOR SUbFIELD CURVES). If $\mathbb{F}_{q}(a, b)=\mathbb{F}_{q^{n}}$, the same arguments as in the non-subfield case apply, and the GHS Weil descent should yield a map $\phi$ whose kernel does not contain the subgroup of order $r$. Let $n^{*}$ be the smallest integer such that $a, b \in \mathbb{F}_{2^{n *}}$. Then, with $q=2^{l}$, we have $\mathbb{F}_{q}(a, b)=\mathbb{F}_{q^{n}}$ if and only if $\operatorname{lcm}\left(n^{*}, l\right)=N$.

For the curves given in Table $2, n^{*}=16$ and $p=N / n^{*}$ is prime. Remarks 22 and 23 imply that we need to analyze exactly those descents from $\mathbb{F}_{q^{n}}$ down to $\mathbb{F}_{q}$ for which $\operatorname{gcd}(n, p)=1$.

We can compute the values of $m$ using formula (5) of Theorem 1 for the various decompositions $N=n l$ without actually performing the GHS reduction. Having computed $m$, and using the fact that $g$ is equal to $2^{m-1}$ or $2^{m-1}-1$, for each decomposition $N=n l$ we can estimate the respective running times for the Enge-Gaudry algorithm as explained in Section 4.2.

Our results for the five ANSI X9.62 curves are listed in Table 3. For all cases where $m<13$, we performed the GHS reductions to determine the exact genera of the resulting hyperelliptic curves; we found that in all cases, $g=2^{m-1}$ (and never that $g=2^{m-1}-1$ ). For each $(n, l, m, g)$, we then computed the optimal smoothness bound $t$, the estimated size $F$ of the factor base, and the corresponding expected running times $T$ and $T_{M}$ for the three cases of the Enge-Gaudry algorithm: with and without the upper bound $10^{7}$ on the factor base size (Cases EG1 and EG2, respectively), and with consideration of the running time for the linear algebra step (Case EG3). For comparison, we list the expected running time $\rho=2 \sqrt{\pi r / N}$ of Pollard's rho method in a subgroup of order $r$ combined with the speedup of $[15,34]$ that is applicable since the elliptic curves are defined over $\mathbb{F}_{216}$. In Table 3 , the entries for $F, T, T_{M}$ and $\rho$ are the logarithms (base 2, rounded to the nearest integer) of the actual values. $D 1$ and $D 2$ denote the differences $\rho-T$ (if positive) for EG1 and EG2, respectively, while $D 3$ denotes the difference $\rho-T_{M}$ (if positive) for EG3. For each curve, the data corresponding to the smallest value of $T$ is given in bold face.

Regardless of the fact that $\phi$ maps points in the large prime-order subgroup of $E\left(\mathbb{F}_{q^{n}}\right)$ to the zero divisor (class) of the resulting Jacobian of the hyperelliptic curve, we determined the attack data also for those descents where $\operatorname{gcd}(n, p)>1$; that is, where $l=2^{i}$ for $i \in\{0,1,2,3,4\}$. These data become relevant should means be found such that $\phi$ does not kill the large prime-order subgroup. We found that, in all except two cases, either the $m$ values are too small for the Jacobians to potentially contain the large prime-order subgroup (see also Remark 25), or the genera of the hyperelliptic curves are larger than $2^{12}-1$, and are thus too large for the resulting HCDLP to be feasible. 
Table 3: GHS attack data for some elliptic curves from ANSI X9.62.

\begin{tabular}{|c|c|c|c|c|c|c|c|c|c|c|c|c|c|c|c|c|c|c|c|c|}
\hline \multirow[b]{2}{*}{$\mathrm{E} N$} & \multirow[b]{2}{*}{$n$} & \multirow[b]{2}{*}{$l$} & \multirow[b]{2}{*}{$m$} & \multirow[b]{2}{*}{$g$} & \multicolumn{5}{|c|}{ EG1 } & \multicolumn{5}{|c|}{ EG2 } & \multicolumn{6}{|c|}{ EG3 } \\
\hline & & & & & $t$ & $F$ & $T$ & $\rho$ & $D 1$ & $t$ & $F$ & $T$ & $\rho$ & $D 2$ & $t$ & $F$ & $T$ & $T_{M}$ & $\rho$ & $D 3$ \\
\hline \multirow[t]{4}{*}{ E176 } & 2 & 88 & 2 & 2 & - & - & - & - & - & 1 & 87 & 88 & 78 & - & 1 & 87 & 88 & 174 & 78 & - \\
\hline & 4 & 44 & 4 & 8 & - & - & - & - & - & 1 & 43 & 58 & 78 & 20 & 1 & 43 & 58 & 86 & 78 & - \\
\hline & 8 & 22 & 8 & 128 & 1 & 21 & 737 & 78 & - & 6 & 128 & 222 & 78 & - & 5 & 107 & 225 & 225 & 78 & - \\
\hline & 16 & 11 & 16 & $2^{15}$ & - & - & - & - & - & - & - & - & - & - & - & - & - & - & - & - \\
\hline \multirow[t]{4}{*}{ E208 } & 2 & 104 & 2 & 2 & - & - & - & - & - & 1 & 103 & 104 & 94 & - & 1 & 103 & 104 & 206 & 94 & - \\
\hline & 4 & 52 & 4 & 8 & - & - & - & - & - & 1 & 51 & 66 & 94 & 28 & 1 & 51 & 66 & 102 & 94 & - \\
\hline & 8 & 26 & 7 & 64 & - & - & - & - & - & 4 & 101 & 161 & 94 & - & 3 & 75 & 162 & 162 & 94 & - \\
\hline & 16 & 13 & 14 & $2^{13}$ & - & - & - & - & - & - & - & - & - & - & - & - & - & - & - & - \\
\hline \multirow[t]{4}{*}{ E272 } & 2 & 136 & 2 & 2 & - & - & - & - & - & 1 & 135 & 136 & 126 & - & 1 & 135 & 136 & 270 & 126 & - \\
\hline & 4 & 68 & 4 & 8 & - & - & - & - & - & 1 & 67 & 82 & 126 & 44 & 1 & 67 & 82 & 134 & 126 & - \\
\hline & 8 & 34 & 8 & 128 & - & - & - & - & - & 5 & 167 & 285 & 126 & - & 4 & 133 & 289 & 289 & 126 & - \\
\hline & 16 & 17 & 16 & $2^{15}$ & - & - & - & - & - & - & - & - & - & - & - & - & - & - & - & - \\
\hline \multirow[t]{4}{*}{ E304 } & 2 & 152 & 2 & 2 & - & - & - & - & - & 1 & 151 & 153 & 142 & - & 1 & 151 & 152 & 302 & 142 & - \\
\hline & 4 & 76 & 4 & 8 & - & - & - & - & - & 1 & 75 & 90 & 142 & 52 & 1 & 75 & 90 & 150 & 142 & - \\
\hline & 8 & 38 & 8 & 128 & - & - & - & - & - & 4 & 149 & 305 & 142 & - & 4 & 149 & 305 & 305 & 142 & - \\
\hline & 16 & 19 & 16 & $2^{15}$ & - & - & - & - & - & - & - & - & - & - & - & - & - & - & - & - \\
\hline \multirow[t]{4}{*}{ E368 } & 2 & 184 & 2 & 2 & - & - & - & - & - & 1 & 183 & 184 & 174 & - & 1 & 183 & 184 & 366 & 174 & - \\
\hline & 4 & 92 & 4 & 8 & - & - & - & - & - & 1 & 91 & 106 & 174 & 68 & 1 & 91 & 106 & 182 & 174 & - \\
\hline & 8 & 46 & 7 & 64 & - & - & - & - & - & 3 & 135 & 222 & 174 & - & 2 & 90 & 229 & 229 & 174 & - \\
\hline & 16 & 23 & 13 & $2^{12}$ & - & - & - & - & - & - & - & - & - & - & - & - & - & - & - & - \\
\hline
\end{tabular}

The two exceptions to this are E176 with $(n, m, g)=(88,8,128)$ and E272 with $(n, m, g)=(136,8,128)$, for which we would have attack data as listed in Table 4 if the GHS descent were not doomed to fail for the reasons given in Remark 22.

Table 4: GHS attack data in two special cases.

\begin{tabular}{rrrrrrrrrrr}
\hline EG1 & E $N$ & $n$ & $l$ & $m$ & $g$ & $t$ & $F$ & $T$ & $\rho$ & $D 1$ \\
\hline & E176 & 88 & 2 & 8 & 128 & 13 & 22 & 54 & 78 & 24 \\
& E272 & 136 & 2 & 8 & 128 & 13 & 22 & 54 & 126 & 72
\end{tabular}

\begin{tabular}{rrrrrrrrrrr}
\hline EG2 & EN & $n$ & $l$ & $m$ & $g$ & $t$ & $F$ & $T$ & $\rho$ & $D 2$ \\
\hline & E176 & 88 & 2 & 8 & 128 & 17 & 29 & 51 & 78 & 27 \\
& E272 & 136 & 2 & 8 & 128 & 17 & 29 & 51 & 126 & 75
\end{tabular}

\begin{tabular}{rrrrrrrrrrrr}
\hline EG3 & EN & $n$ & $l$ & $m$ & $g$ & $t$ & $F$ & $T$ & $T_{M}$ & $\rho$ & $D 3$ \\
\hline & E176 & 88 & 2 & 8 & 128 & 15 & 26 & 52 & 52 & 78 & 26 \\
& E272 & 136 & 2 & 8 & 128 & 15 & 26 & 52 & 52 & 126 & 74 \\
\hline
\end{tabular}


REMARK 24 (FAILURE OF THE GHS ATtACK FOR Ei76 AND E272). When evaluating the mapping $\phi: E\left(\mathbb{F}_{2^{176}}\right) \rightarrow J_{C}\left(\mathbb{F}_{2^{2}}\right)$ (where $E=$ E176) constructed by the GHS attack, we found that the large subgroup $\langle P\rangle$ of prime order $r \approx 2^{160}$ is indeed contained in the kernel of $\phi$, and is thus of no use for solving ECDLPs in $E\left(\mathbb{F}_{2} 176\right)$. The same situation was observed with the mapping $\phi: E\left(\mathbb{F}_{2^{272}}\right) \rightarrow J_{C}\left(\mathbb{F}_{2^{2}}\right)$ for $E=\mathrm{E} 272$.

ReMARK 25 ( $m$ VAlues FOR THE CASES $l=1,2,4,8,16$ ). Suppose that $l \in\{1,2,4,8,16\}$, and let $\sigma: \alpha \mapsto \alpha^{2^{l}}$ be the Frobenius map on $\mathbb{F}_{2^{N}}$. Then, since $b \in \mathbb{F}_{2^{16}} \backslash \mathbb{F}_{2^{8}}$, we have $(\sigma+1)^{16 / l} b=0$ but $(\sigma+1)^{8 / l} b \neq 0$. Thus we expect that $8 / l<m \leqslant 16 / l$.

REMARK 26 (APPLICABILITy OF THE GHS REDUCTION). ForE176, E272 andE304, we have $\operatorname{Tr}_{K / \mathbb{F}_{2}}(a)=1$, so that condition (6) of Theorem 1 is not satisfied for these curves whenever $m(b) \neq n$. However, the weaker condition (8) of Lemma 6 does hold, and that is why the GHS reduction does produce hyperelliptic curves of genus $2^{m-1}$ or $2^{m-1}-1$ over $\mathbb{F}_{q}$, even when $m \neq n$.

REMARK 27 (EXISTENCE OFISOGENOUSCURVESTHAT MAY YIELDEASIER HCDLPINSTANCES). To exclude the applicability of the extended GHS attack (see [14] and Remark 20), we checked whether any of the ANSI X9.62 curves are isogenous to an elliptic curve for which the GHS reduction produces an easier HCDLP instance. For this, we use a modification of Algorithm 13 that allows the elliptic curve to be defined over a proper subfield $\mathbb{F}_{2^{l \mu}}$ of $\mathbb{F}_{2^{N}}$ with $l \mu \leqslant 16$ if and only if $\operatorname{gcd}(n, p)=1$ (see Remarks 22 and 23). That is, if $\operatorname{gcd}(n, p)=1$, in Algorithm 13 we accept $m$ even if Corollary 10 applies, as long as $l \mu \leqslant 16$.

Since this time we are interested not only in the best instance $(N, n, m)$, but in any instance for which the GHS attack yields an algorithm that is more efficient than Pollard rho, we give the estimated running times for all decompositions $N=n l$ in Table 5. The notation is the same as in Table 1 . Observe that with the single exception of $(N, n, m)=$ $(208,208,13)$, for all the parameters listed here curves exist that are defined over the full field $\mathbb{F}_{q^{n}}$ and no proper subfield of it.

(i) EI76. The only possibility to improve on the GHS attack highlighted in Table 3 is to find a curve that is isogenous to $\mathrm{E} 176$, and for which $(n, m)=(8,5)$. Since there are $I \approx 2^{110}$ isomorphism classes of curves over $\mathbb{F}_{2^{176}}$ with these parameters, it is quite possible that such a curve exists. However, finding such a curve seems to be harder than solving the ECDLP using Pollard rho.

(ii) E208. When allowing a factor base up to $2^{25}$ elements, we could improve on the GHS attack highlighted in Table 3. However, similar to the case of E176, it does not seem feasible to find a curve isogenous to E208 with $(n, m)=(8,5)$ from among $2^{130}$ isomorphism classes.

(iii) E272. The best option would be to find a curve isogenous to E272 with $(n, m)=(8,5)$. But even if working with a factor base of size $2^{33}$ were feasible, finding such a curve from among the $2^{170}$ isomorphism classes seems well beyond the realm of feasibility.

(iv) E304. For the same reasons as for E272, it is not possible to improve on the GHS attack using isogenies.

(v) E368. This case is the same as E304. 
Table 5: Extended GHS attack data for the ANSI X9.62 elliptic curves.

\begin{tabular}{|c|c|c|c|c|c|c|c|c|c|c|c|c|c|c|c|c|c|c|c|c|c|}
\hline \multirow[b]{2}{*}{$\mathrm{E} N$} & \multirow[b]{2}{*}{$n$} & \multirow[b]{2}{*}{$l$} & \multirow[b]{2}{*}{$m$} & \multirow[b]{2}{*}{$g$} & \multirow[b]{2}{*}{$I$} & \multicolumn{5}{|c|}{ EG1 } & \multicolumn{5}{|c|}{ EG2 } & \multicolumn{6}{|c|}{ EG3 } \\
\hline & & & & & & $t$ & $F$ & $T$ & $\rho$ & $D 1$ & $t$ & $F$ & $T$ & $\rho$ & $D 2$ & $t$ & $F$ & $T$ & $T_{M}$ & $\rho$ & $D 3$ \\
\hline \multirow[t]{9}{*}{ E176 } & 2 & 88 & 2 & 2 & 177 & - & - & - & - & - & 1 & 87 & 88 & 78 & - & 1 & 87 & 88 & 174 & 78 & - \\
\hline & 4 & 44 & 3 & 4 & 132 & - & - & - & - & - & 1 & 43 & 48 & 78 & 30 & 1 & 43 & 48 & 86 & 78 & - \\
\hline & 8 & 22 & 5 & 15 & 110 & 1 & 21 & 61 & 78 & 17 & 2 & 42 & 59 & 78 & 19 & 1 & 21 & 61 & 61 & 78 & 17 \\
\hline & 11 & 16 & - & - & - & - & - & - & - & - & - & - & - & - & - & - & - & - & - & - & - \\
\hline & 16 & 11 & 9 & 255 & 99 & 2 & 20 & 839 & 78 & - & 12 & 127 & 225 & 78 & - & 10 & 106 & 230 & 230 & 78 & - \\
\hline & 22 & 8 & - & - & - & - & - & - & - & - & - & - & - & - & - & - & - & - & - & - & - \\
\hline & 44 & 4 & - & - & - & - & - & - & - & - & - & - & - & - & - & - & - & - & - & - & - \\
\hline & 88 & 2 & - & - & - & - & - & - & - & - & - & - & - & - & - & - & - & - & - & - & - \\
\hline & 176 & 1 & - & - & - & - & - & - & - & - & - & - & - & - & - & - & - & - & - & - & - \\
\hline \multirow[t]{9}{*}{ E208 } & 2 & 104 & 2 & 2 & 209 & - & - & - & - & - & 1 & 103 & 104 & 94 & - & 1 & 103 & 104 & 206 & 94 & - \\
\hline & 4 & 52 & 3 & 4 & 156 & - & - & - & - & - & 1 & 51 & 56 & 94 & 38 & 1 & 51 & 56 & 102 & 94 & - \\
\hline & 8 & 26 & 5 & 15 & 130 & - & - & - & - & - & 1 & 25 & 65 & 94 & 29 & 1 & 25 & 65 & 65 & 94 & 29 \\
\hline & 13 & 16 & - & - & - & - & - & - & - & - & - & - & - & - & - & - & - & - & - & - & - \\
\hline & 16 & 13 & 9 & 255 & 117 & 1 & 12 & 1688 & 94 & - & 11 & 139 & 248 & 94 & - & 10 & 126 & 250 & 252 & 94 & - \\
\hline & 26 & 8 & - & - & - & - & - & - & - & - & - & - & - & - & - & - & - & - & - & - & - \\
\hline & 52 & 4 & - & - & - & - & - & - & - & - & - & - & - & - & - & - & - & - & - & - & - \\
\hline & 104 & 2 & - & - & - & - & - & - & - & - & - & - & - & - & - & - & - & - & - & - & - \\
\hline & 208 & 1 & 13 & 4095 & 14 & 28 & 23 & 1208 & 94 & - & 120 & 113 & 307 & 94 & - & 120 & 113 & 307 & 307 & 94 & - \\
\hline \multirow[t]{9}{*}{ E272 } & 2 & 136 & 2 & 2 & 273 & - & - & - & - & - & 1 & 135 & 136 & 126 & - & 1 & 135 & 136 & 270 & 126 & - \\
\hline & 4 & 68 & 3 & 4 & 204 & - & - & - & - & - & 1 & 67 & 72 & 126 & 54 & 1 & 67 & 72 & 134 & 126 & - \\
\hline & 8 & 34 & 5 & 15 & 170 & - & - & - & - & - & 1 & 33 & 73 & 126 & 53 & 1 & 33 & 73 & 73 & 126 & 53 \\
\hline & 16 & 17 & - & - & - & - & - & - & - & - & - & - & - & - & - & - & - & - & - & - & - \\
\hline & 17 & 16 & 9 & 255 & 146 & 1 & 15 & 1691 & 126 & - & 10 & 156 & 280 & 126 & - & 9 & 140 & 282 & 282 & 126 & - \\
\hline & 34 & 8 & 10 & 511 & 82 & 3 & 21 & 1260 & 126 & - & 21 & 163 & 283 & 126 & - & 18 & 139 & 286 & 286 & 126 & - \\
\hline & 68 & 4 & 11 & 1023 & 45 & 6 & 21 & 1352 & 126 & - & 42 & 162 & 284 & 126 & - & 37 & 142 & 287 & 287 & 126 & - \\
\hline & 136 & 2 & - & - & - & - & - & - & - & - & - & - & - & - & - & - & - & - & - & - & - \\
\hline & 272 & 1 & - & - & - & - & - & - & - & - & - & - & - & - & - & - & - & - & - & - & - \\
\hline \multirow[t]{9}{*}{ E304 } & 2 & 152 & 2 & 2 & 305 & - & - & - & - & - & 1 & 151 & 152 & 142 & - & 1 & 151 & 152 & 302 & 142 & - \\
\hline & 4 & 76 & 3 & 4 & 228 & - & - & - & - & - & 1 & 75 & 80 & 142 & 62 & 1 & 75 & 80 & 150 & 142 & - \\
\hline & 8 & 38 & 5 & 15 & 190 & - & - & - & - & - & 1 & 37 & 77 & 142 & 65 & 1 & 37 & 77 & 77 & 142 & 65 \\
\hline & 16 & 19 & - & - & - & - & - & - & - & - & - & - & - & - & - & - & - & - & - & - & - \\
\hline & 19 & 16 & - & - & - & - & - & - & - & - & - & - & - & - & - & - & - & - & - & - & - \\
\hline & 38 & 8 & - & - & - & - & - & - & - & - & - & - & - & - & - & - & - & - & - & - & - \\
\hline & 76 & 4 & - & - & - & - & - & - & - & - & - & - & - & - & - & - & - & - & - & - & - \\
\hline & 152 & 2 & - & - & - & - & - & - & - & - & - & - & - & - & - & - & - & - & - & - & - \\
\hline & 304 & 1 & - & - & - & - & - & - & - & - & - & - & - & - & - & - & - & - & - & - & - \\
\hline \multirow[t]{9}{*}{ E368 } & 2 & 184 & 2 & 2 & 369 & - & - & - & - & - & 1 & 183 & 184 & 174 & - & 1 & 183 & 184 & 366 & 174 & - \\
\hline & 4 & 92 & 3 & 4 & 276 & - & - & - & - & - & 1 & 91 & 96 & 174 & 78 & 1 & 91 & 96 & 182 & 174 & - \\
\hline & 8 & 46 & 5 & 15 & 230 & - & - & - & - & - & 1 & 45 & 85 & 174 & 89 & 1 & 45 & 85 & 90 & 174 & 84 \\
\hline & 16 & 23 & - & - & - & - & - & - & - & - & - & - & - & - & - & - & - & - & - & - & - \\
\hline & 23 & 16 & - & - & - & - & - & - & - & - & - & - & - & - & - & - & - & - & - & - & - \\
\hline & 46 & 8 & - & - & - & - & - & - & - & - & - & - & - & - & - & - & - & - & - & - & - \\
\hline & 92 & 4 & - & - & - & - & - & - & - & - & - & - & - & - & - & - & - & - & - & - & - \\
\hline & 184 & 2 & - & - & - & - & - & - & - & - & - & - & - & - & - & - & - & - & - & - & - \\
\hline & 368 & 1 & - & - & - & - & - & - & - & - & - & - & - & - & - & - & - & - & - & - & - \\
\hline
\end{tabular}


Our analysis shows that the extended GHS attack does not yield a faster algorithm to solve the ECDLP for the ANSI X9.62 curves. However, once the problem of finding an isogenous curve from among the most vulnerable isomorphism classes identified above can be solved more efficiently, the GHS attack takes significantly fewer steps than Pollard's rho algorithm.

\section{ECDLP challenges}

We present some cryptographically interesting ECDLP instances that we hope will help stimulate interest in both computational and theoretical work on the ECDLP, Weil descent, and the HCDLP. Appendix B provides details on how the ECDLP instances were generated verifiably at random in such a way that the solutions were not known to us a priori. The ECDLP instances themselves, as well as the hyperelliptic curves and divisors produced by invoking Hess's KASH program [18] for performing the GHS reduction, are presented in Appendix C. The remainder of this section provides a rationale for the choice of elliptic curves.

The cryptographically interesting elliptic curves E161, E180, E186, E217, E248 and E300 were specially selected from the class of elliptic curves over $\mathbb{F}_{2161}, \mathbb{F}_{2^{180}}, \mathbb{F}_{2^{186}}, \mathbb{F}_{2^{217}}$, $\mathbb{F}_{2248}$ and $\mathbb{F}_{2^{300}}$, respectively, for which the GHS attack yields HCDLP instances that are within reach of the Enge-Gaudry algorithm. Furthermore, Pollard's rho algorithm for solving the ECDLP on these elliptic curves is infeasible. E186, E217 and E248 are extensions of the E62, E93, E124 and E155 series of elliptic curves analyzed in [20]: these are elliptic curves defined over $\mathbb{F}_{2^{31 l}}$ for which the GHS attack yields a genus 31 hyperelliptic curve over $\mathbb{F}_{2^{l}}$. The low genus of 31 is possible because the multiplicative order of 2 modulo 31 is small (see Theorem 11).

Table 6 lists the $(n, l, g)$ GHS attack parameters that yield HCDLP instances that can be solved in $\approx 2^{T_{M}}$ steps using a smoothness bound of $t$ and a factor base of size $\approx 2^{F}$. Note that $T_{M} \ll \rho$, where $2^{\rho}$ is the approximate time taken to solve an ECDLP instance using Pollard's rho algorithm. The Enge-Gaudry parameters $(t, F, T)$ were selected to minimize the running time $T_{M}$. Table 7 illustrates how the factor base size, the expected number of random-walk steps $\left(\approx 2^{E}\right)$ to find a smooth divisor and the total expected number of random-walk steps depend on the smoothness bound $t$. The ECDLP in E161 is expected to be a little easier than the ECDLP in the E155 curve of [20], which has $T_{M}=37$. The latter problem was concluded to be tractable in [20], on the basis of experimental data gathered by solving the ECDLP in E62, E93 and E124.

We emphasize that these ECDLP challenge problems may become more tractable if advances are made in index-calculus methods for the HCDLP, or in techniques for solving large systems of sparse linear equations. Another avenue for improvement is to apply the Weil descent methodology to map the ECDLP efficiently to the DLP in abelian varieties (not necessarily hyperelliptic), which are easier to solve that the HCDLP instances produced by the GHS attack. For an illustration of this possibility, see [2], where Weil descent is used to reduce the ECDLP in elliptic curves over characteristic three finite fields to the DLP in $C_{a b}$ curves. See also [7] for a study on Weil restriction.

The E176 and E272 elliptic curves are from ANSI X9.62. As discussed in Section 6, the GHS reduction maps these elliptic curves to hyperelliptic curves of genus 128 over $\mathbb{F}_{2^{2}}$, where the HCDLP is feasible. However, the large prime-order subgroup is mapped to the zero divisor, since for both curves, $\mathbb{F}_{2^{2}}(a, b)=\mathbb{F}_{2^{16}} \neq \mathbb{F}_{q^{n}}$. It is an open problem whether and how the GHS attack could be modified in this case so that the resulting map does not kill the large prime order subgroup. Diem [7, Proposition 3.13] shows how Weil descent 
Table 6: GHS attack parameters for the challenge curves.

\begin{tabular}{rrrrrrrrrr}
\hline Curve & $N$ & $n$ & $l$ & $g$ & $t$ & $F$ & $T$ & $T_{M}$ & $\rho$ \\
\hline E161 & 161 & 7 & 23 & 7 & 1 & 22 & 34 & 44 & 80 \\
E180 & 180 & 30 & 6 & 31 & 4 & 21 & 41 & 42 & 89 \\
E186 & 186 & 31 & 6 & 31 & 4 & 21 & 41 & 42 & 92 \\
E217 & 217 & 31 & 7 & 31 & 3 & 18 & 49 & 49 & 108 \\
E248 & 248 & 31 & 8 & 31 & 3 & 21 & 52 & 52 & 123 \\
E300 & 300 & 30 & 10 & 31 & 3 & 27 & 58 & 58 & 149 \\
\hline E176 & 176 & 88 & 2 & 128 & 15 & 26 & 52 & 52 & 78 \\
E272 & 272 & 136 & 2 & 128 & 15 & 26 & 52 & 52 & 126 \\
\hline E161-2 & 161 & 7 & 23 & 64 & 3 & 66 & 153 & 153 & 80 \\
\hline
\end{tabular}

Table 7: Some Enge-Gaudry parameters for the challenge curves.

\begin{tabular}{|c|c|c|c|c|c|c|c|c|c|c|c|c|c|c|c|}
\hline \multicolumn{16}{|c|}{ E161 } \\
\hline$t$ & 1 & 2 & 3 & 4 & 5 & 6 & 7 & 8 & 9 & 10 & 11 & 12 & 13 & 14 & 15 \\
\hline$F$ & 22 & 44 & 66 & 89 & 112 & 134 & 157 & 180 & 203 & 226 & 249 & 271 & 294 & 317 & 340 \\
\hline$E$ & 12 & 4 & 2 & 1 & 1 & 0 & 0 & 0 & 0 & 0 & 0 & 0 & 0 & 0 & 0 \\
\hline$T$ & 34 & 48 & 68 & 90 & 112 & 135 & 157 & 180 & 203 & 226 & 249 & 271 & 294 & 317 & 340 \\
\hline \multicolumn{16}{|c|}{ E180 } \\
\hline$t$ & 1 & 2 & 3 & 4 & 5 & 6 & 7 & 8 & 9 & 10 & 11 & 12 & 13 & 14 & 15 \\
\hline$F$ & 11 & 22 & 33 & 45 & 57 & 68 & 80 & 92 & 104 & 116 & 128 & 139 & 151 & 163 & 175 \\
\hline$E$ & 40 & 17 & 9 & 6 & 4 & 3 & 2 & 1 & 1 & 1 & 1 & 0 & 0 & 0 & 0 \\
\hline$T$ & 51 & 39 & 43 & 51 & 61 & 71 & 82 & 93 & 105 & 116 & 128 & 140 & 152 & 163 & 175 \\
\hline \multicolumn{16}{|c|}{ E186 } \\
\hline$t$ & 1 & 2 & 3 & 4 & 5 & 6 & 7 & 8 & 9 & 10 & 11 & 12 & 13 & 14 & 15 \\
\hline$F$ & 5 & 10 & 15 & 21 & 27 & 32 & 38 & 44 & 50 & 56 & 62 & 67 & 73 & 79 & 85 \\
\hline$E$ & 109 & 51 & 30 & 20 & 15 & 11 & 8 & 7 & 5 & 4 & 4 & 3 & 3 & 2 & 2 \\
\hline$T$ & 114 & 61 & 46 & 41 & 41 & 43 & 47 & 51 & 55 & 60 & 65 & 70 & 76 & 81 & 87 \\
\hline \multicolumn{16}{|c|}{ E217 } \\
\hline$t$ & 1 & 2 & 3 & 4 & 5 & 6 & 7 & 8 & 9 & 10 & 11 & 12 & 13 & 14 & 15 \\
\hline$F$ & 6 & 12 & 18 & 25 & 32 & 38 & 45 & 52 & 59 & 66 & 73 & 79 & 86 & 93 & 100 \\
\hline$E$ & 112 & 51 & 30 & 20 & 15 & 11 & 8 & 7 & 5 & 4 & 4 & 3 & 3 & 2 & 2 \\
\hline$T$ & 118 & 63 & 49 & 45 & 46 & 49 & 54 & 59 & 64 & 70 & 76 & 82 & 89 & 95 & 102 \\
\hline \multicolumn{16}{|c|}{ E248 } \\
\hline$t$ & 1 & 2 & 3 & 4 & 5 & 6 & 7 & 8 & 9 & 10 & 11 & 12 & 13 & 14 & 15 \\
\hline$F$ & 7 & 14 & 21 & 29 & 37 & 44 & 52 & 60 & 68 & 76 & 84 & 91 & 99 & 107 & 115 \\
\hline$E$ & 112 & 51 & 30 & 20 & 15 & 11 & 8 & 7 & 5 & 4 & 4 & 3 & 3 & 2 & 2 \\
\hline$T$ & 119 & 65 & 52 & 49 & 51 & 55 & 61 & 67 & 73 & 80 & 87 & 94 & 102 & 109 & 117 \\
\hline \multicolumn{16}{|c|}{ E300 } \\
\hline$t$ & 1 & 2 & 3 & 4 & 5 & 6 & 7 & 8 & 9 & 10 & 11 & 12 & 13 & 14 & 15 \\
\hline$F$ & 19 & 38 & 57 & 77 & 97 & 116 & 136 & 156 & 176 & 196 & 216 & 235 & 255 & 275 & 295 \\
\hline$E$ & 40 & 17 & 9 & 6 & 4 & 3 & 2 & 1 & 1 & 1 & 1 & 0 & 0 & 0 & 0 \\
\hline$T$ & 59 & 55 & 67 & 83 & 101 & 119 & 138 & 157 & 177 & 196 & 216 & 236 & 256 & 275 & 295 \\
\hline
\end{tabular}


could be applied to reduce an ECDLP in an elliptic curve $E\left(\mathbb{F}_{2^{p t}}\right)$ defined over $\mathbb{F}_{2^{t}}$ to a DLP in the group $\mathrm{Cl}^{0}(C)$ of divisor classes of degree zero of a curve $C$ of genus $\leqslant 2^{2 t}-1$ defined over $\mathbb{F}_{2^{2 t}}$. Here, $p$ is an odd prime, and $t=\operatorname{ord}_{2}(p)$ denotes the order of 2 modulo $p$. For example, an elliptic curve over $\mathbb{F}_{2^{136}}$ defined over $\mathbb{F}_{2^{8}}$ could be transformed to the DLP in $\mathrm{Cl}^{0}(C)$ of a curve $C$ of genus $\leqslant 2^{16}-1$ defined over $\mathbb{F}_{2^{16}}$; however, Diem's result does not apply to E176 or E272.

Finally, the E161-2 elliptic curve was generated at random from the set of all cryptographically interesting elliptic curves over $\mathbb{F}_{2^{161}}$ (see Appendix B). The GHS reduction yielded $(m, g)=(7,64)$ for $(n, l)=(7,23), m=23$ for $(n, l)=(23,7)$, and $m=158$ for $(n, l)=(161,1)$. All three resulting HCDLPs are outside the realm of feasibility of the Enge-Gaudry algorithm. However, from the results in [14] (see Remark 20), it is likely that there exists an elliptic curve $E^{\prime}$ over $\mathbb{F}_{2161}$ that is isogenous to E161-2, and for which the GHS reduction produces a hyperelliptic curve of genus 7 over $\mathbb{F}_{2^{23}}$ in which the HCDLP is feasible. If such an elliptic curve $E^{\prime}$ could be found (this is no easy task, since there are approximately $2^{94}$ isomorphism classes of elliptic curves over $\mathbb{F}_{2^{161}}$ with $m=4$ for $n=7$ ), then the isogeny could be computed using the algorithm in [14].

\section{Conclusions}

We analyzed the GHS Weil descent attack on the ECDLP for elliptic curves defined over characteristic two finite fields $\mathbb{F}_{2^{N}}$ of composite extension degree $N \in[100,600]$. For some such fields, there are cryptographically interesting elliptic curves over $\mathbb{F}_{2^{N}}$ where the ECDLP succumbs to the GHS attack. We provided ECDLP 'challenges' over six such fields: $\mathbb{F}_{2^{161}}, \mathbb{F}_{2^{180}}, \mathbb{F}_{2^{186}}, \mathbb{F}_{2^{217}}, \mathbb{F}_{2^{248}}$ and $\mathbb{F}_{2^{300}}$. For other such fields $\mathbb{F}_{2^{N}}$, our results demonstrate that there are no cryptographically interesting elliptic curves over $\mathbb{F}_{2^{N}}$ for which the GHS attack yields an ECDLP solver that is faster than Pollard's rho method. Our analysis suggests that the five elliptic curves over $\mathbb{F}_{2^{176}}, \mathbb{F}_{2^{208}}, \mathbb{F}_{2^{272}}, \mathbb{F}_{2^{304}}$ and $\mathbb{F}_{2^{368}}$ in ANSI X9.62 resist the GHS attack.

We stress that any statement we have made regarding the failure of the GHS attack on some elliptic curves over some field $\mathbb{F}_{2^{N}}$ is dependent on the assumption that the EngeGaudry algorithm cannot be significantly improved. Also, we stress that failure of the GHS attack does not imply failure of the Weil descent methodology - there may be other useful curves that lie on the Weil restriction $W_{E / k}$, but that were not constructed by the GHS method. We thus hope that our work can serve as a stimulus for further work on the Weil descent method, on subexponential-time index-calculus methods for the HCDLP, and on algorithms for solving large systems of sparse linear equations.

Acknowledgements. We used KASH, Magma, Maple and NTL in our work. We would like to thank Steven Galbraith, Florian Hess, Antoine Joux, David McKinnon, Nigel Smart and an anonymous referee for their helpful advice and comments. Thanks in particular to Florian Hess for explaining to us the contents of Remark 22, and to Antoine Joux for answering our questions about solving large systems of sparse linear equations. We would also like to thank NSERC for providing partial funding for this research.

\section{Appendix A. Results of our analysis}

For an explanation of the notation used in the following tables, see Section 5. 
Table 8: Results of our analysis

\begin{tabular}{|c|c|c|c|c|c|c|c|c|c|c|c|c|c|c|c|c|c|c|c|c|c|c|c|c|c|c|c|c|c|c|c|}
\hline \multirow[b]{2}{*}{$N$} & \multicolumn{10}{|c|}{ EG1 } & \multicolumn{10}{|c|}{ EG2 } & \multicolumn{11}{|c|}{ EG3 } \\
\hline & $n$ & $l$ & $m$ & $g$ & $I$ & $t$ & $F$ & $T$ & $\rho$ & $D 1$ & $n$ & $l$ & $m$ & $g$ & $I$ & $t$ & $F$ & $T$ & $\rho$ & $D 2$ & $n$ & $l$ & $m$ & $g$ & $I$ & $t$ & $F$ & $T$ & $T_{M}$ & $\rho$ & D3 \\
\hline 100 & 5 & 20 & 5 & 15 & 101 & 1 & 19 & 59 & 49 & - & 4 & 25 & 3 & 4 & 75 & 1 & 24 & 29 & 49 & 20 & 4 & 25 & 3 & 4 & 75 & 1 & 24 & 29 & 48 & 49 & 1 \\
\hline 102 & 6 & 17 & 4 & 7 & 69 & 1 & 16 & 28 & 50 & 22 & 6 & 17 & 4 & 7 & 69 & 1 & 16 & 28 & 50 & 22 & 6 & 17 & 4 & 7 & 69 & 1 & 16 & 28 & 32 & 50 & 18 \\
\hline 104 & 8 & 13 & 5 & 15 & 65 & 1 & 12 & 52 & 51 & - & 4 & 26 & 3 & 4 & 78 & 1 & 25 & 30 & 51 & 21 & 8 & 13 & 5 & 15 & 65 & 2 & 24 & 41 & 48 & 51 & 3 \\
\hline 105 & 7 & 15 & 4 & 7 & 62 & 1 & 14 & 26 & 52 & 26 & 7 & 15 & 4 & 7 & 62 & 1 & 14 & 26 & 52 & 26 & 7 & 15 & 4 & 7 & 62 & 1 & 14 & 26 & 28 & 52 & 24 \\
\hline 106 & - & - & - & - & - & - & - & - & - & - & 2 & 53 & 2 & 2 & 107 & 1 & 52 & 53 & 52 & - & 2 & 53 & 2 & 2 & 107 & 1 & 52 & 53 & 104 & 52 & - \\
\hline 108 & 6 & 18 & 4 & 7 & 73 & 1 & 17 & 29 & 53 & 24 & 6 & 18 & 4 & 7 & 73 & 1 & 17 & 29 & 53 & 24 & 12 & 9 & 5 & 15 & 45 & 2 & 16 & 33 & 33 & 53 & 20 \\
\hline 110 & 5 & 22 & 5 & 15 & 111 & 1 & 21 & 61 & 54 & - & 2 & 55 & 2 & 2 & 111 & 1 & 54 & 55 & 54 & - & 5 & 22 & 5 & 15 & 111 & 1 & 21 & 61 & 61 & 54 & - \\
\hline 111 & - & - & - & - & - & - & - & - & - & - & 3 & 37 & 3 & 3 & 112 & 1 & 36 & 39 & 55 & 16 & 3 & 37 & 3 & 3 & 112 & 1 & 36 & 39 & 72 & 55 & - \\
\hline 112 & 7 & 16 & 4 & 7 & 66 & 1 & 15 & 27 & 55 & 28 & 7 & 16 & 4 & 7 & 66 & 1 & 15 & 27 & 55 & 28 & 7 & 16 & 4 & 7 & 66 & 1 & 15 & 27 & 30 & 55 & 25 \\
\hline 114 & 6 & 19 & 4 & 7 & 77 & 1 & 18 & 30 & 56 & 26 & 6 & 19 & 4 & 7 & 77 & 1 & 18 & 30 & 56 & 26 & 6 & 19 & 4 & 7 & 77 & 1 & 18 & 30 & 36 & 56 & 20 \\
\hline 115 & 5 & 23 & 5 & 15 & 116 & 1 & 22 & 62 & 57 & - & 5 & 23 & 5 & 15 & 116 & 2 & 44 & 61 & 57 & - & 5 & 23 & 5 & 15 & 116 & 1 & 22 & 62 & 62 & 57 & - \\
\hline 116 & - & - & - & - & - & - & - & - & - & - & 4 & 29 & 3 & 4 & 87 & 1 & 28 & 33 & 57 & 24 & 4 & 29 & 3 & 4 & 87 & 1 & 28 & 33 & 56 & 57 & 1 \\
\hline 117 & 9 & 13 & 7 & 63 & 92 & 1 & 12 & 302 & 58 & - & 3 & 39 & 3 & 3 & 118 & 1 & 38 & 41 & 58 & 17 & 3 & 39 & 3 & 3 & 118 & 1 & 38 & 41 & 76 & 58 & - \\
\hline 118 & - & - & - & - & - & - & - & - & - & - & 2 & 59 & 2 & 2 & 119 & 1 & 58 & 59 & 58 & - & 2 & 59 & 2 & 2 & 119 & 1 & 58 & 59 & 116 & 58 & - \\
\hline 119 & 7 & 17 & 4 & 7 & 70 & 1 & 16 & 28 & 59 & 31 & 7 & 17 & 4 & 7 & 70 & 1 & 16 & 28 & 59 & 31 & 7 & 17 & 4 & 7 & 70 & 1 & 16 & 28 & 32 & 59 & 27 \\
\hline 120 & 6 & 20 & 4 & 7 & 81 & 1 & 19 & 31 & 59 & 28 & 6 & 20 & 4 & 7 & 81 & 1 & 19 & 31 & 59 & 28 & 15 & 8 & 5 & 15 & 42 & 2 & 14 & 31 & 31 & 59 & 28 \\
\hline 121 & - & - & - & - & - & - & - & - & - & - & - & - & - & - & - & - & - & - & - & - & - & - & - & - & - & - & - & - & - & - & - \\
\hline 122 & - & - & - & - & - & - & - & - & - & - & 2 & 61 & 2 & 2 & 123 & 1 & 60 & 61 & 60 & - & 2 & 61 & 2 & 2 & 123 & 1 & 60 & 61 & 120 & 60 & - \\
\hline 123 & - & - & - & - & - & - & - & - & - & - & 3 & 41 & 3 & 3 & 124 & 1 & 40 & 43 & 61 & 18 & 3 & 41 & 3 & 3 & 124 & 1 & 40 & 43 & 80 & 61 & - \\
\hline 124 & 31 & 4 & 6 & 31 & 28 & 5 & 17 & 31 & 61 & 30 & 31 & 4 & 6 & 31 & 28 & 5 & 17 & 31 & 61 & 30 & 62 & 2 & 7 & 63 & 17 & 10 & 16 & 32 & 32 & 61 & 29 \\
\hline 125 & - & - & - & - & - & - & - & - & - & - & 5 & 25 & 5 & 15 & 126 & 1 & 24 & 64 & 62 & - & 5 & 25 & 5 & 15 & 126 & 1 & 24 & 64 & 64 & 62 & - \\
\hline 126 & 7 & 18 & 4 & 7 & 74 & 1 & 17 & 29 & 62 & 33 & 7 & 18 & 4 & 7 & 74 & 1 & 17 & 29 & 62 & 33 & 63 & 2 & 7 & 63 & 18 & 10 & 16 & 32 & 32 & 62 & 30 \\
\hline 128 & 8 & 16 & 5 & 15 & 80 & 1 & 15 & 55 & 63 & 8 & 4 & 32 & 3 & 4 & 96 & 1 & 31 & 36 & 63 & 27 & 8 & 16 & 5 & 15 & 80 & 1 & 15 & 55 & 55 & 63 & 8 \\
\hline 129 & - & - & - & - & - & - & - & - & - & - & 3 & 43 & 3 & 3 & 130 & 1 & 42 & 45 & 64 & 19 & 3 & 43 & 3 & 3 & 130 & 1 & 42 & 45 & 84 & 64 & - \\
\hline 130 & 10 & 13 & 6 & 31 & 79 & 1 & 12 & 125 & 64 & - & 2 & 65 & 2 & 2 & 131 & 1 & 64 & 65 & 64 & - & 5 & 26 & 5 & 15 & 131 & 1 & 25 & 65 & 65 & 64 & - \\
\hline 132 & 6 & 22 & 4 & 7 & 89 & 1 & 21 & 33 & 65 & 32 & 6 & 22 & 4 & 7 & 89 & 1 & 21 & 33 & 65 & 32 & 12 & 11 & 5 & 15 & 55 & 2 & 20 & 37 & 40 & 65 & 25 \\
\hline 133 & 7 & 19 & 4 & 7 & 78 & 1 & 18 & 30 & 66 & 36 & 7 & 19 & 4 & 7 & 78 & 1 & 18 & 30 & 66 & 36 & 7 & 19 & 4 & 7 & 78 & 1 & 18 & 30 & 36 & 66 & 30 \\
\hline 134 & - & - & - & - & - & - & - & - & - & - & 2 & 67 & 2 & 2 & 135 & 1 & 66 & 67 & 66 & - & 2 & 67 & 2 & 2 & 135 & 1 & 66 & 67 & 132 & 66 & - \\
\hline
\end{tabular}




\begin{tabular}{|c|c|c|c|c|c|c|c|c|c|c|c|c|c|c|c|c|c|c|c|c|c|c|c|c|c|c|c|c|c|c|c|}
\hline \multirow[b]{2}{*}{$N$} & \multicolumn{10}{|c|}{ EG1 } & \multicolumn{10}{|c|}{ EG2 } & \multicolumn{11}{|c|}{ EG3 } \\
\hline & $n$ & $l$ & $m$ & $g$ & $I$ & $t$ & $F$ & $T$ & $\rho$ & $D 1$ & $n$ & $l$ & $m$ & $g$ & $I$ & $t$ & $F$ & $T$ & $\rho$ & $D 2$ & $n$ & $l$ & $m$ & $g$ & $I$ & $t$ & $F$ & $T$ & $T_{M}$ & $\rho$ & D3 \\
\hline 135 & 15 & 9 & 5 & 15 & 47 & 2 & 16 & 33 & 67 & 34 & 15 & 9 & 5 & 15 & 47 & 2 & 16 & 33 & 67 & 34 & 15 & 9 & 5 & 15 & 47 & 2 & 16 & 33 & 33 & 67 & 34 \\
\hline 136 & 8 & 17 & 5 & 15 & 85 & 1 & 16 & 56 & 67 & 11 & 4 & 34 & 3 & 4 & 102 & 1 & 33 & 38 & 67 & 29 & 8 & 17 & 5 & 15 & 85 & 1 & 16 & 56 & 56 & 67 & 11 \\
\hline 138 & 6 & 23 & 4 & 7 & 93 & 1 & 22 & 34 & 68 & 34 & 6 & 23 & 4 & 7 & 93 & 1 & 22 & 34 & 68 & 34 & 6 & 23 & 4 & 7 & 93 & 1 & 22 & 34 & 44 & 68 & 24 \\
\hline 140 & 7 & 20 & 4 & 7 & 82 & 1 & 19 & 31 & 69 & 38 & 7 & 20 & 4 & 7 & 82 & 1 & 19 & 31 & 69 & 38 & 14 & 10 & 5 & 15 & 52 & 2 & 18 & 35 & 36 & 69 & 33 \\
\hline 141 & - & - & - & - & - & - & - & - & - & - & 3 & 47 & 3 & 3 & 142 & 1 & 46 & 49 & 70 & 21 & 3 & 47 & 3 & 3 & 142 & 1 & 46 & 49 & 92 & 70 & - \\
\hline 142 & - & - & - & - & - & - & - & - & - & - & 2 & 71 & 2 & 2 & 143 & 1 & 70 & 71 & 70 & - & 2 & 71 & 2 & 2 & 143 & 1 & 70 & 71 & 140 & 70 & - \\
\hline 143 & - & - & - & - & - & - & - & - & - & - & - & - & - & - & - & - & - & - & - & - & - & - & - & - & - & - & - & - & - & - & - \\
\hline 144 & 6 & 24 & 4 & 7 & 97 & 1 & 23 & 35 & 71 & 36 & 6 & 24 & 4 & 7 & 97 & 1 & 23 & 35 & 71 & 36 & 12 & 12 & 5 & 15 & 60 & 2 & 22 & 39 & 44 & 71 & 27 \\
\hline 145 & - & - & - & - & - & - & - & - & - & - & 5 & 29 & 5 & 15 & 146 & 1 & 28 & 68 & 72 & 4 & 5 & 29 & 5 & 15 & 146 & 1 & 28 & 68 & 68 & 72 & 4 \\
\hline 146 & 146 & 1 & 11 & 1023 & 14 & 28 & 23 & 232 & 72 & - & 2 & 73 & 2 & 2 & 147 & 1 & 72 & 73 & 72 & - & 73 & 2 & 10 & 511 & 24 & 34 & 62 & 125 & 125 & 72 & - \\
\hline 147 & 7 & 21 & 4 & 7 & 86 & 1 & 20 & 32 & 73 & 41 & 7 & 21 & 4 & 7 & 86 & 1 & 20 & 32 & 73 & 41 & 7 & 21 & 4 & 7 & 86 & 1 & 20 & 32 & 40 & 73 & 33 \\
\hline 148 & - & - & - & - & - & - & - & - & - & - & 4 & 37 & 3 & 4 & 111 & 1 & 36 & 41 & 73 & 32 & 4 & 37 & 3 & 4 & 111 & 1 & 36 & 41 & 72 & 73 & 1 \\
\hline 150 & 15 & 10 & 5 & 15 & 52 & 2 & 18 & 35 & 74 & 39 & 15 & 10 & 5 & 15 & 52 & 2 & 18 & 35 & 74 & 39 & 15 & 10 & 5 & 15 & 52 & 2 & 18 & 35 & 36 & 74 & 38 \\
\hline 152 & 8 & 19 & 5 & 15 & 95 & 1 & 18 & 58 & 75 & 17 & 4 & 38 & 3 & 4 & 114 & 1 & 37 & 42 & 75 & 33 & 8 & 19 & 5 & 15 & 95 & 1 & 18 & 58 & 58 & 75 & 17 \\
\hline 153 & 51 & 3 & 9 & 255 & 30 & 9 & 23 & 165 & 76 & - & 3 & 51 & 3 & 3 & 154 & 1 & 50 & 53 & 76 & 23 & 3 & 51 & 3 & 3 & 154 & 1 & 50 & 53 & 100 & 76 & - \\
\hline 154 & 7 & 22 & 4 & 7 & 90 & 1 & 21 & 33 & 76 & 43 & 7 & 22 & 4 & 7 & 90 & 1 & 21 & 33 & 76 & 43 & 14 & 11 & 5 & 15 & 57 & 2 & 20 & 37 & 40 & 76 & 36 \\
\hline 155 & 31 & 5 & 6 & 31 & 34 & 5 & 22 & 36 & 77 & 41 & 31 & 5 & 6 & 31 & 34 & 5 & 22 & 36 & 77 & 41 & 31 & 5 & 6 & 31 & 34 & 4 & 17 & 37 & 37 & 77 & 40 \\
\hline 156 & 12 & 13 & 5 & 15 & 65 & 1 & 12 & 52 & 77 & 25 & 6 & 26 & 4 & 7 & 105 & 1 & 25 & 37 & 77 & 40 & 12 & 13 & 5 & 15 & 65 & 2 & 24 & 41 & 48 & 77 & 29 \\
\hline 158 & - & - & - & - & - & - & - & - & - & - & 2 & 79 & 2 & 2 & 159 & 1 & 78 & 79 & 78 & - & 2 & 79 & 2 & 2 & 159 & 1 & 78 & 79 & 156 & 78 & - \\
\hline 159 & - & - & - & - & - & - & - & - & - & - & 3 & 53 & 3 & 3 & 160 & 1 & 52 & 55 & 79 & 24 & 3 & 53 & 3 & 3 & 160 & 1 & 52 & 55 & 104 & 79 & - \\
\hline 160 & 8 & 20 & 5 & 15 & 100 & 1 & 19 & 59 & 79 & 20 & 4 & 40 & 3 & 4 & 120 & 1 & 39 & 44 & 79 & 35 & 8 & 20 & 5 & 15 & 100 & 1 & 19 & 59 & 59 & 79 & 20 \\
\hline 161 & 7 & 23 & 4 & 7 & 94 & 1 & 22 & 34 & 80 & 46 & 7 & 23 & 4 & 7 & 94 & 1 & 22 & 34 & 80 & 46 & 7 & 23 & 4 & 7 & 94 & 1 & 22 & 34 & 44 & 80 & 36 \\
\hline 162 & 9 & 18 & 7 & 63 & 127 & 1 & 17 & 307 & 80 & - & 6 & 27 & 4 & 7 & 109 & 1 & 26 & 38 & 80 & 42 & 6 & 27 & 4 & 7 & 109 & 1 & 26 & 38 & 52 & 80 & 28 \\
\hline 164 & - & - & - & - & - & - & - & - & - & - & 4 & 41 & 3 & 4 & 123 & 1 & 40 & 45 & 81 & 36 & 4 & 41 & 3 & 4 & 123 & 1 & 40 & 45 & 80 & 81 & 1 \\
\hline 165 & 15 & 11 & 5 & 15 & 57 & 2 & 20 & 37 & 82 & 45 & 15 & 11 & 5 & 15 & 57 & 2 & 20 & 37 & 82 & 45 & 15 & 11 & 5 & 15 & 57 & 2 & 20 & 37 & 40 & 82 & 42 \\
\hline 166 & - & - & - & - & - & - & - & - & - & - & 2 & 83 & 2 & 2 & 167 & 1 & 82 & 83 & 82 & - & 2 & 83 & 2 & 2 & 167 & 1 & 82 & 83 & 164 & 82 & - \\
\hline 168 & 7 & 24 & 4 & 7 & 98 & 1 & 23 & 35 & 83 & 48 & 7 & 24 & 4 & 7 & 98 & 1 & 23 & 35 & 83 & 48 & 28 & 6 & 6 & 31 & 37 & 4 & 21 & 41 & 42 & 83 & 41 \\
\hline 169 & - & - & - & - & - & - & - & - & - & - & - & - & - & - & - & - & - & - & - & - & - & - & - & - & - & - & - & - & - & - & - \\
\hline
\end{tabular}




\begin{tabular}{|c|c|c|c|c|c|c|c|c|c|c|c|c|c|c|c|c|c|c|c|c|c|c|c|c|c|c|c|c|c|c|c|}
\hline \multirow[b]{2}{*}{$N$} & \multicolumn{10}{|c|}{ EG1 } & \multicolumn{10}{|c|}{ EG2 } & \multicolumn{11}{|c|}{ EG3 } \\
\hline & $n$ & $l$ & $m$ & $g$ & $I$ & $t$ & $F$ & $T$ & $\rho$ & $D 1$ & $n$ & $l$ & $m$ & $g$ & I & $t$ & $F$ & $T$ & $\rho$ & $D 2$ & $n$ & $l$ & $m$ & $g$ & $I$ & $t$ & $F$ & $T$ & $T_{M}$ & $\rho$ & D3 \\
\hline 170 & 170 & 1 & 10 & 511 & 13 & 28 & 23 & 105 & 84 & - & 5 & 34 & 5 & 15 & 171 & 1 & 33 & 73 & 84 & 11 & 5 & 34 & 5 & 15 & 171 & 1 & 33 & 73 & 73 & 84 & 11 \\
\hline 171 & 9 & 19 & 7 & 63 & 134 & 1 & 18 & 308 & 85 & - & 3 & 57 & 3 & 3 & 172 & 1 & 56 & 59 & 85 & 26 & 3 & 57 & 3 & 3 & 172 & 1 & 56 & 59 & 112 & 85 & - \\
\hline 172 & - & - & - & - & - & - & - & - & - & - & 4 & 43 & 3 & 4 & 129 & 1 & 42 & 47 & 85 & 38 & 4 & 43 & 3 & 4 & 129 & 1 & 42 & 47 & 84 & 85 & 1 \\
\hline 174 & - & - & - & - & - & - & - & - & - & - & 6 & 29 & 4 & 7 & 117 & 1 & 28 & 40 & 86 & 46 & 6 & 29 & 4 & 7 & 117 & 1 & 28 & 40 & 56 & 86 & 30 \\
\hline 175 & 35 & 5 & 8 & 127 & 42 & 5 & 22 & 139 & 87 & - & 7 & 25 & 4 & 7 & 102 & 1 & 24 & 36 & 87 & 51 & 7 & 25 & 4 & 7 & 102 & 1 & 24 & 36 & 48 & 87 & 39 \\
\hline 176 & 8 & 22 & 5 & 15 & 110 & 1 & 21 & 61 & 87 & 26 & 4 & 44 & 3 & 4 & 132 & 1 & 43 & 48 & 87 & 39 & 8 & 22 & 5 & 15 & 110 & 1 & 21 & 61 & 61 & 87 & 26 \\
\hline 177 & - & - & - & - & - & - & - & - & - & - & 3 & 59 & 3 & 3 & 178 & 1 & 58 & 61 & 88 & 27 & 3 & 59 & 3 & 3 & 178 & 1 & 58 & 61 & 116 & 88 & - \\
\hline 178 & 178 & 1 & 13 & 4095 & 16 & 28 & 23 & 1208 & 88 & - & 2 & 89 & 2 & 2 & 179 & 1 & 88 & 89 & 88 & - & 2 & 89 & 2 & 2 & 179 & 1 & 88 & 89 & 176 & 88 & - \\
\hline 180 & 15 & 12 & 5 & 15 & 62 & 2 & 22 & 39 & 89 & 50 & 15 & 12 & 5 & 15 & 62 & 2 & 22 & 39 & 89 & 50 & 30 & 6 & 6 & 31 & 38 & 4 & 21 & 41 & 42 & 89 & 47 \\
\hline 182 & 14 & 13 & 5 & 15 & 67 & 1 & 12 & 52 & 90 & 38 & 7 & 26 & 4 & 7 & 106 & 1 & 25 & 37 & 90 & 53 & 14 & 13 & 5 & 15 & 67 & 2 & 24 & 41 & 48 & 90 & 42 \\
\hline 183 & - & - & - & - & - & - & - & - & - & - & 3 & 61 & 3 & 3 & 184 & 1 & 60 & 63 & 91 & 28 & 3 & 61 & 3 & 3 & 184 & 1 & 60 & 63 & 120 & 91 & - \\
\hline 184 & 8 & 23 & 5 & 15 & 115 & 1 & 22 & 62 & 91 & 29 & 4 & 46 & 3 & 4 & 138 & 1 & 45 & 50 & 91 & 41 & 8 & 23 & 5 & 15 & 115 & 1 & 22 & 62 & 62 & 91 & 29 \\
\hline 185 & - & - & - & - & - & - & - & - & - & - & 5 & 37 & 5 & 15 & 186 & 1 & 36 & 76 & 92 & 16 & 5 & 37 & 5 & 15 & 186 & 1 & 36 & 76 & 76 & 92 & 16 \\
\hline 186 & 31 & 6 & 6 & 31 & 40 & 4 & 21 & 41 & 92 & 51 & 31 & 6 & 6 & 31 & 40 & 5 & 27 & 41 & 92 & 51 & 31 & 6 & 6 & 31 & 40 & 4 & 21 & 41 & 42 & 92 & 50 \\
\hline 187 & 17 & 11 & 9 & 255 & 101 & 2 & 20 & 839 & 93 & - & 17 & 11 & 9 & 255 & 101 & 12 & 127 & 225 & 93 & - & 17 & 11 & 9 & 255 & 101 & 10 & 106 & 230 & 230 & 93 & - \\
\hline 188 & - & - & - & - & - & - & - & - & - & - & 4 & 47 & 3 & 4 & 141 & 1 & 46 & 51 & 93 & 42 & 4 & 47 & 3 & 4 & 141 & 1 & 46 & 51 & 92 & 93 & 1 \\
\hline 189 & 63 & 3 & 7 & 63 & 25 & 9 & 23 & 42 & 94 & 52 & 7 & 27 & 4 & 7 & 110 & 1 & 26 & 38 & 94 & 56 & 63 & 3 & 7 & 63 & 25 & 8 & 20 & 43 & 43 & 94 & 51 \\
\hline 190 & 10 & 19 & 6 & 31 & 115 & 1 & 18 & 131 & 94 & - & 5 & 38 & 5 & 15 & 191 & 1 & 37 & 77 & 94 & 17 & 5 & 38 & 5 & 15 & 191 & 1 & 37 & 77 & 77 & 94 & 17 \\
\hline 192 & 12 & 16 & 5 & 15 & 80 & 1 & 15 & 55 & 95 & 40 & 6 & 32 & 4 & 7 & 129 & 1 & 31 & 43 & 95 & 52 & 12 & 16 & 5 & 15 & 80 & 1 & 15 & 55 & 55 & 95 & 40 \\
\hline 194 & - & - & - & - & - & - & - & - & - & - & 2 & 97 & 2 & 2 & 195 & 1 & 96 & 97 & 96 & - & 2 & 97 & 2 & 2 & 195 & 1 & 96 & 97 & 192 & 96 & - \\
\hline 195 & 15 & 13 & 5 & 15 & 67 & 1 & 12 & 52 & 97 & 45 & 15 & 13 & 5 & 15 & 67 & 2 & 24 & 41 & 97 & 56 & 15 & 13 & 5 & 15 & 67 & 2 & 24 & 41 & 48 & 97 & 49 \\
\hline 196 & 28 & 7 & 6 & 31 & 43 & 3 & 18 & 49 & 97 & 48 & 7 & 28 & 4 & 7 & 114 & 1 & 27 & 39 & 97 & 58 & 28 & 7 & 6 & 31 & 43 & 3 & 18 & 49 & 49 & 97 & 48 \\
\hline 198 & 9 & 22 & 7 & 63 & 155 & 1 & 21 & 311 & 98 & - & 6 & 33 & 4 & 7 & 133 & 1 & 32 & 44 & 98 & 54 & 6 & 33 & 4 & 7 & 133 & 1 & 32 & 44 & 64 & 98 & 34 \\
\hline 200 & 10 & 20 & 6 & 31 & 121 & 1 & 19 & 132 & 99 & - & 4 & 50 & 3 & 4 & 150 & 1 & 49 & 54 & 99 & 45 & 8 & 25 & 5 & 15 & 125 & 1 & 24 & 64 & 64 & 99 & 35 \\
\hline 201 & - & - & - & - & - & - & - & - & - & - & 3 & 67 & 3 & 3 & 202 & 1 & 66 & 69 & 100 & 31 & 3 & 67 & 3 & 3 & 202 & 1 & 66 & 69 & 132 & 100 & - \\
\hline 202 & - & - & - & - & - & - & - & - & - & - & 2 & 101 & 2 & 2 & 203 & 1 & 100 & 101 & 100 & - & 2 & 101 & 2 & 2 & 203 & 1 & 100 & 101 & 200 & 100 & - \\
\hline 203 & - & - & - & - & - & - & - & - & - & - & 7 & 29 & 4 & 7 & 118 & 1 & 28 & 40 & 101 & 61 & 7 & 29 & 4 & 7 & 118 & 1 & 28 & 40 & 56 & 101 & 45 \\
\hline 204 & 12 & 17 & 5 & 15 & 85 & 1 & 16 & 56 & 101 & 45 & 6 & 34 & 4 & 7 & 137 & 1 & 33 & 45 & 101 & 56 & 12 & 17 & 5 & 15 & 85 & 1 & 16 & 56 & 56 & 101 & 45 \\
\hline
\end{tabular}




\begin{tabular}{|c|c|c|c|c|c|c|c|c|c|c|c|c|c|c|c|c|c|c|c|c|c|c|c|c|c|c|c|c|c|c|c|}
\hline \multirow[b]{2}{*}{$N$} & \multicolumn{10}{|c|}{ EG1 } & \multicolumn{10}{|c|}{ EG2 } & \multicolumn{11}{|c|}{ EG3 } \\
\hline & $n$ & $l$ & $m$ & $g$ & $I$ & $t$ & $F$ & $T$ & $\rho$ & $D 1$ & $n$ & $l$ & $m$ & $g$ & $I$ & $t$ & $F$ & $T$ & $\rho$ & $D 2$ & $n$ & $l$ & $m$ & $g$ & $I$ & $t$ & $F$ & $T$ & $T_{M}$ & $\rho$ & D3 \\
\hline 205 & - & - & - & - & - & - & - & - & - & - & 5 & 41 & 5 & 15 & 206 & 1 & 40 & 80 & 102 & 22 & 5 & 41 & 5 & 15 & 206 & 1 & 40 & 80 & 80 & 102 & 22 \\
\hline 206 & - & - & - & - & - & - & - & - & - & - & 2 & 103 & 2 & 2 & 207 & 1 & 102 & 103 & 102 & - & 2 & 103 & 2 & 2 & 207 & 1 & 102 & 103 & 204 & 102 & - \\
\hline 207 & 9 & 23 & 7 & 63 & 162 & 1 & 22 & 312 & 103 & - & 3 & 69 & 3 & 3 & 208 & 1 & 68 & 71 & 103 & 32 & 3 & 69 & 3 & 3 & 208 & 1 & 68 & 71 & 136 & 103 & - \\
\hline 208 & 16 & 13 & 9 & 255 & 117 & 1 & 12 & 1688 & 103 & - & 4 & 52 & 3 & 4 & 156 & 1 & 51 & 56 & 103 & 47 & 8 & 26 & 5 & 15 & 130 & 1 & 25 & 65 & 65 & 103 & 38 \\
\hline 209 & - & - & - & - & - & - & - & - & - & - & - & - & - & - & - & - & - & - & - & - & - & - & - & - & - & - & - & - & - & - & - \\
\hline 210 & 30 & 7 & 6 & 31 & 44 & 3 & 18 & 49 & 104 & 55 & 7 & 30 & 4 & 7 & 122 & 1 & 29 & 41 & 104 & 63 & 30 & 7 & 6 & 31 & 44 & 3 & 18 & 49 & 49 & 104 & 55 \\
\hline 212 & - & - & - & - & - & - & - & - & - & - & 4 & 53 & 3 & 4 & 159 & 1 & 52 & 57 & 105 & 48 & 4 & 53 & 3 & 4 & 159 & 1 & 52 & 57 & 104 & 105 & 1 \\
\hline 213 & - & - & - & - & - & - & - & - & - & - & 3 & 71 & 3 & 3 & 214 & 1 & 70 & 73 & 106 & 33 & 3 & 71 & 3 & 3 & 214 & 1 & 70 & 73 & 140 & 106 & - \\
\hline 214 & - & - & - & - & - & - & - & - & - & - & 2 & 107 & 2 & 2 & 215 & 1 & 106 & 107 & 106 & - & 2 & 107 & 2 & 2 & 215 & 1 & 106 & 107 & 212 & 106 & - \\
\hline 215 & - & - & - & - & - & - & - & - & - & - & 5 & 43 & 5 & 15 & 216 & 1 & 42 & 82 & 107 & 25 & 5 & 43 & 5 & 15 & 216 & 1 & 42 & 82 & 84 & 107 & 23 \\
\hline 216 & 12 & 18 & 5 & 15 & 90 & 1 & 17 & 57 & 107 & 50 & 6 & 36 & 4 & 7 & 145 & 1 & 35 & 47 & 107 & 60 & 12 & 18 & 5 & 15 & 90 & 1 & 17 & 57 & 57 & 107 & 50 \\
\hline 217 & 31 & 7 & 6 & 31 & 46 & 3 & 18 & 49 & 108 & 59 & 7 & 31 & 4 & 7 & 126 & 1 & 30 & 42 & 108 & 66 & 31 & 7 & 6 & 31 & 46 & 3 & 18 & 49 & 49 & 108 & 59 \\
\hline 218 & - & - & - & - & - & - & - & - & - & - & 2 & 109 & 2 & 2 & 219 & 1 & 108 & 109 & 108 & - & 2 & 109 & 2 & 2 & 219 & 1 & 108 & 109 & 216 & 108 & - \\
\hline 219 & 73 & 3 & 10 & 511 & 34 & 9 & 23 & 375 & 109 & - & 3 & 73 & 3 & 3 & 220 & 1 & 72 & 75 & 109 & 34 & 3 & 73 & 3 & 3 & 220 & 1 & 72 & 75 & 144 & 109 & - \\
\hline 220 & 10 & 22 & 6 & 31 & 133 & 1 & 21 & 134 & 109 & - & 4 & 55 & 3 & 4 & 165 & 1 & 54 & 59 & 109 & 50 & 5 & 44 & 5 & 15 & 221 & 1 & 43 & 83 & 86 & 109 & 23 \\
\hline 221 & 17 & 13 & 9 & 255 & 119 & 1 & 12 & 1688 & 110 & - & 17 & 13 & 9 & 255 & 119 & 11 & 139 & 248 & 110 & - & 17 & 13 & 9 & 255 & 119 & 10 & 126 & 250 & 252 & 110 & - \\
\hline 222 & - & - & - & - & - & - & - & - & - & - & 6 & 37 & 4 & 7 & 149 & 1 & 36 & 48 & 110 & 62 & 6 & 37 & 4 & 7 & 149 & 1 & 36 & 48 & 72 & 110 & 38 \\
\hline 224 & 28 & 8 & 6 & 31 & 49 & 3 & 21 & 52 & 111 & 59 & 7 & 32 & 4 & 7 & 130 & 1 & 31 & 43 & 111 & 68 & 28 & 8 & 6 & 31 & 49 & 3 & 21 & 52 & 52 & 111 & 59 \\
\hline 225 & 15 & 15 & 5 & 15 & 77 & 1 & 14 & 54 & 112 & 58 & 15 & 15 & 5 & 15 & 77 & 2 & 28 & 45 & 112 & 67 & 15 & 15 & 5 & 15 & 77 & 1 & 14 & 54 & 54 & 112 & 58 \\
\hline 226 & - & - & - & - & - & - & - & - & - & - & 2 & 113 & 2 & 2 & 227 & 1 & 112 & 113 & 112 & - & 2 & 113 & 2 & 2 & 227 & 1 & 112 & 113 & 224 & 112 & - \\
\hline 228 & 12 & 19 & 5 & 15 & 95 & 1 & 18 & 58 & 113 & 55 & 6 & 38 & 4 & 7 & 153 & 1 & 37 & 49 & 113 & 64 & 12 & 19 & 5 & 15 & 95 & 1 & 18 & 58 & 58 & 113 & 55 \\
\hline 230 & 10 & 23 & 6 & 31 & 139 & 1 & 22 & 135 & 114 & - & 5 & 46 & 5 & 15 & 231 & 1 & 45 & 85 & 114 & 29 & 5 & 46 & 5 & 15 & 231 & 1 & 45 & 85 & 90 & 114 & 24 \\
\hline 231 & 21 & 11 & 6 & 31 & 68 & 2 & 20 & 71 & 115 & 44 & 7 & 33 & 4 & 7 & 134 & 1 & 32 & 44 & 115 & 71 & 21 & 11 & 6 & 31 & 68 & 3 & 30 & 61 & 61 & 115 & 54 \\
\hline 232 & - & - & - & - & - & - & - & - & - & - & 4 & 58 & 3 & 4 & 174 & 1 & 57 & 62 & 115 & 53 & 8 & 29 & 5 & 15 & 145 & 1 & 28 & 68 & 68 & 115 & 47 \\
\hline 234 & 18 & 13 & 8 & 127 & 105 & 1 & 12 & 721 & 116 & - & 6 & 39 & 4 & 7 & 157 & 1 & 38 & 50 & 116 & 66 & 6 & 39 & 4 & 7 & 157 & 1 & 38 & 50 & 76 & 116 & 40 \\
\hline 235 & - & - & - & - & - & - & - & - & - & - & 5 & 47 & 5 & 15 & 236 & 1 & 46 & 86 & 117 & 31 & 5 & 47 & 5 & 15 & 236 & 1 & 46 & 86 & 92 & 117 & 25 \\
\hline 236 & - & - & - & - & - & - & - & - & - & - & 4 & 59 & 3 & 4 & 177 & 1 & 58 & 63 & 117 & 54 & 4 & 59 & 3 & 4 & 177 & 1 & 58 & 63 & 116 & 117 & 1 \\
\hline 237 & - & - & - & - & - & - & - & - & - & - & 3 & 79 & 3 & 3 & 238 & 1 & 78 & 81 & 118 & 37 & 3 & 79 & 3 & 3 & 238 & 1 & 78 & 81 & 156 & 118 & - \\
\hline
\end{tabular}




\begin{tabular}{|c|c|c|c|c|c|c|c|c|c|c|c|c|c|c|c|c|c|c|c|c|c|c|c|c|c|c|c|c|c|c|c|}
\hline \multirow[b]{2}{*}{$N$} & \multicolumn{10}{|c|}{ EG1 } & \multicolumn{10}{|c|}{ EG2 } & \multicolumn{11}{|c|}{ EG3 } \\
\hline & $n$ & $l$ & $m$ & $g$ & $I$ & $t$ & $F$ & $T$ & $\rho$ & $D 1$ & $n$ & $l$ & $m$ & $g$ & $I$ & $t$ & $F$ & $T$ & $\rho$ & $D 2$ & $n$ & $l$ & $m$ & $g$ & $I$ & $t$ & $F$ & $T$ & $T_{M}$ & $\rho$ & $D 3$ \\
\hline 238 & 14 & 17 & 5 & 15 & 87 & 1 & 16 & 56 & 118 & 62 & 7 & 34 & 4 & 7 & 138 & 1 & 33 & 45 & 118 & 73 & 14 & 17 & 5 & 15 & 87 & 1 & 16 & 56 & 56 & 118 & 62 \\
\hline 240 & 30 & 8 & 6 & 31 & 50 & 3 & 21 & 52 & 119 & 67 & 15 & 16 & 5 & 15 & 82 & 2 & 30 & 47 & 119 & 72 & 30 & 8 & 6 & 31 & 50 & 3 & 21 & 52 & 52 & 119 & 67 \\
\hline 242 & - & - & - & - & - & - & - & - & - & - & 2 & 121 & 2 & 2 & 243 & 1 & 120 & 121 & 120 & - & 2 & 121 & 2 & 2 & 243 & 1 & 120 & 121 & 240 & 120 & - \\
\hline 243 & - & - & - & - & - & - & - & - & - & - & 3 & 81 & 3 & 3 & 244 & 1 & 80 & 83 & 121 & 38 & 3 & 81 & 3 & 3 & 244 & 1 & 80 & 83 & 160 & 121 & - \\
\hline 244 & - & - & - & - & - & - & - & - & - & - & 4 & 61 & 3 & 4 & 183 & 1 & 60 & 65 & 121 & 56 & 4 & 61 & 3 & 4 & 183 & 1 & 60 & 65 & 120 & 121 & 1 \\
\hline 245 & 35 & 7 & 8 & 127 & 58 & 3 & 18 & 235 & 122 & - & 7 & 35 & 4 & 7 & 142 & 1 & 34 & 46 & 122 & 76 & 7 & 35 & 4 & 7 & 142 & 1 & 34 & 46 & 68 & 122 & 54 \\
\hline 246 & - & - & - & - & - & - & - & - & - & - & 6 & 41 & 4 & 7 & 165 & 1 & 40 & 52 & 122 & 70 & 6 & 41 & 4 & 7 & 165 & 1 & 40 & 52 & 80 & 122 & 42 \\
\hline 247 & - & - & - & - & - & - & - & - & - & - & - & - & - & - & - & - & - & - & - & - & - & - & - & - & - & - & - & - & - & - & - \\
\hline 248 & 31 & 8 & 6 & 31 & 52 & 3 & 21 & 52 & 123 & 71 & 31 & 8 & 6 & 31 & 52 & 4 & 29 & 49 & 123 & 74 & 31 & 8 & 6 & 31 & 52 & 3 & 21 & 52 & 52 & 123 & 71 \\
\hline 249 & - & - & - & - & - & - & - & - & - & - & 3 & 83 & 3 & 3 & 250 & 1 & 82 & 85 & 124 & 39 & 3 & 83 & 3 & 3 & 250 & 1 & 82 & 85 & 164 & 124 & - \\
\hline 250 & - & - & - & - & - & - & - & - & - & - & 5 & 50 & 5 & 15 & 251 & 1 & 49 & 89 & 124 & 35 & 5 & 50 & 5 & 15 & 251 & 1 & 49 & 89 & 98 & 124 & 26 \\
\hline 252 & 252 & 1 & 9 & 255 & 11 & 28 & 23 & 53 & 125 & 72 & 7 & 36 & 4 & 7 & 146 & 1 & 35 & 47 & 125 & 78 & 63 & 4 & 7 & 63 & 32 & 7 & 24 & 52 & 52 & 125 & 73 \\
\hline 253 & - & - & - & - & - & - & - & - & - & - & - & - & - & - & - & - & - & - & - & - & - & - & - & - & - & - & - & - & - & - & - \\
\hline 254 & 254 & 1 & 9 & 255 & 13 & 28 & 23 & 53 & 126 & 73 & 127 & 2 & 8 & 127 & 21 & 17 & 29 & 51 & 126 & 75 & 127 & 2 & 8 & 127 & 21 & 15 & 26 & 51 & 52 & 126 & 74 \\
\hline 255 & 255 & 1 & 9 & 255 & 14 & 28 & 23 & 53 & 127 & 74 & 15 & 17 & 5 & 15 & 87 & 2 & 32 & 49 & 127 & 78 & 255 & 1 & 9 & 255 & 14 & 30 & 25 & 52 & 52 & 127 & 75 \\
\hline 256 & 16 & 16 & 9 & 255 & 144 & 1 & 15 & 1691 & 127 & - & 4 & 64 & 3 & 4 & 192 & 1 & 63 & 68 & 127 & 59 & 8 & 32 & 5 & 15 & 160 & 1 & 31 & 71 & 71 & 127 & 56 \\
\hline 258 & - & - & - & - & - & - & - & - & - & - & 6 & 43 & 4 & 7 & 173 & 1 & 42 & 54 & 128 & 74 & 6 & 43 & 4 & 7 & 173 & 1 & 42 & 54 & 84 & 128 & 44 \\
\hline 259 & - & - & - & - & - & - & - & - & - & - & 7 & 37 & 4 & 7 & 150 & 1 & 36 & 48 & 129 & 81 & 7 & 37 & 4 & 7 & 150 & 1 & 36 & 48 & 72 & 129 & 57 \\
\hline 260 & 20 & 13 & 7 & 63 & 91 & 1 & 12 & 302 & 129 & - & 4 & 65 & 3 & 4 & 195 & 1 & 64 & 69 & 129 & 60 & 10 & 26 & 6 & 31 & 157 & 2 & 50 & 101 & 101 & 129 & 28 \\
\hline 261 & - & - & - & - & - & - & - & - & - & - & 3 & 87 & 3 & 3 & 262 & 1 & 86 & 89 & 130 & 41 & 9 & 29 & 7 & 63 & 204 & 3 & 84 & 169 & 169 & 130 & - \\
\hline 262 & - & - & - & - & - & - & - & - & - & - & 2 & 131 & 2 & 2 & 263 & 1 & 130 & 131 & 130 & - & 2 & 131 & 2 & 2 & 263 & 1 & 130 & 131 & 260 & 130 & - \\
\hline 264 & 12 & 22 & 5 & 15 & 110 & 1 & 21 & 61 & 131 & 70 & 6 & 44 & 4 & 7 & 177 & 1 & 43 & 55 & 131 & 76 & 12 & 22 & 5 & 15 & 110 & 1 & 21 & 61 & 61 & 131 & 70 \\
\hline 265 & - & - & - & - & - & - & - & - & - & - & 5 & 53 & 5 & 15 & 266 & 1 & 52 & 92 & 132 & 40 & 5 & 53 & 5 & 15 & 266 & 1 & 52 & 92 & 104 & 132 & 28 \\
\hline 266 & 14 & 19 & 5 & 15 & 97 & 1 & 18 & 58 & 132 & 74 & 7 & 38 & 4 & 7 & 154 & 1 & 37 & 49 & 132 & 83 & 14 & 19 & 5 & 15 & 97 & 1 & 18 & 58 & 58 & 132 & 74 \\
\hline 267 & - & - & - & - & - & - & - & - & - & - & 3 & 89 & 3 & 3 & 268 & 1 & 88 & 91 & 133 & 42 & 3 & 89 & 3 & 3 & 268 & 1 & 88 & 91 & 176 & 133 & - \\
\hline 268 & - & - & - & - & - & - & - & - & - & - & 4 & 67 & 3 & 4 & 201 & 1 & 66 & 71 & 133 & 62 & 4 & 67 & 3 & 4 & 201 & 1 & 66 & 71 & 132 & 133 & 1 \\
\hline 270 & 15 & 18 & 5 & 15 & 92 & 1 & 17 & 57 & 134 & 77 & 15 & 18 & 5 & 15 & 92 & 2 & 34 & 51 & 134 & 83 & 30 & 9 & 6 & 31 & 56 & 3 & 24 & 55 & 55 & 134 & 79 \\
\hline 272 & 34 & 8 & 10 & 511 & 82 & 3 & 21 & 1260 & 135 & - & 4 & 68 & 3 & 4 & 204 & 1 & 67 & 72 & 135 & 63 & 8 & 34 & 5 & 15 & 170 & 1 & 33 & 73 & 73 & 135 & 62 \\
\hline
\end{tabular}




\begin{tabular}{|c|c|c|c|c|c|c|c|c|c|c|c|c|c|c|c|c|c|c|c|c|c|c|c|c|c|c|c|c|c|c|c|}
\hline \multirow[b]{2}{*}{$N$} & \multicolumn{10}{|c|}{ EG1 } & \multicolumn{10}{|c|}{ EG2 } & \multicolumn{11}{|c|}{ EG3 } \\
\hline & $n$ & $l$ & $m$ & $g$ & $I$ & $t$ & $F$ & $T$ & $\rho$ & $D 1$ & $n$ & $l$ & $m$ & $g$ & $I$ & $t$ & $F$ & $T$ & $\rho$ & D2 & $n$ & $l$ & $m$ & $g$ & $I$ & $t$ & $F$ & $T$ & $T_{M}$ & $\rho$ & D3 \\
\hline 273 & 21 & 13 & 6 & 31 & 80 & 1 & 12 & 125 & 136 & 11 & 7 & 39 & 4 & 7 & 158 & 1 & 38 & 50 & 136 & 86 & 21 & 13 & 6 & 31 & 80 & 3 & 36 & 67 & 72 & 136 & 64 \\
\hline 274 & - & - & - & - & - & - & - & - & - & - & 2 & 137 & 2 & 2 & 275 & 1 & 136 & 137 & 136 & - & 2 & 137 & 2 & 2 & 275 & 1 & 136 & 137 & 272 & 136 & - \\
\hline 275 & - & - & - & - & - & - & - & - & - & - & 5 & 55 & 5 & 15 & 276 & 1 & 54 & 94 & 137 & 43 & 5 & 55 & 5 & 15 & 276 & 1 & 54 & 94 & 108 & 137 & 29 \\
\hline 276 & 12 & 23 & 5 & 15 & 115 & 1 & 22 & 62 & 137 & 75 & 6 & 46 & 4 & 7 & 185 & 1 & 45 & 57 & 137 & 80 & 12 & 23 & 5 & 15 & 115 & 1 & 22 & 62 & 62 & 137 & 75 \\
\hline 278 & - & - & - & - & - & - & - & - & - & - & 2 & 139 & 2 & 2 & 279 & 1 & 138 & 139 & 138 & - & 2 & 139 & 2 & 2 & 279 & 1 & 138 & 139 & 276 & 138 & - \\
\hline 279 & 31 & 9 & 6 & 31 & 58 & 2 & 16 & 67 & 139 & 72 & 31 & 9 & 6 & 31 & 58 & 4 & 33 & 53 & 139 & 86 & 31 & 9 & 6 & 31 & 58 & 3 & 24 & 55 & 55 & 139 & 84 \\
\hline 280 & 14 & 20 & 5 & 15 & 102 & 1 & 19 & 59 & 139 & 80 & 7 & 40 & 4 & 7 & 162 & 1 & 39 & 51 & 139 & 88 & 28 & 10 & 6 & 31 & 61 & 3 & 27 & 58 & 58 & 139 & 81 \\
\hline 282 & - & - & - & - & - & - & - & - & - & - & 6 & 47 & 4 & 7 & 189 & 1 & 46 & 58 & 140 & 82 & 6 & 47 & 4 & 7 & 189 & 1 & 46 & 58 & 92 & 140 & 48 \\
\hline 284 & - & - & - & - & - & - & - & - & - & - & 4 & 71 & 3 & 4 & 213 & 1 & 70 & 75 & 141 & 66 & 4 & 71 & 3 & 4 & 213 & 1 & 70 & 75 & 140 & 141 & 1 \\
\hline 285 & 15 & 19 & 5 & 15 & 97 & 1 & 18 & 58 & 142 & 84 & 15 & 19 & 5 & 15 & 97 & 2 & 36 & 53 & 142 & 89 & 15 & 19 & 5 & 15 & 97 & 1 & 18 & 58 & 58 & 142 & 84 \\
\hline 286 & - & - & - & - & - & - & - & - & - & - & 2 & 143 & 2 & 2 & 287 & 1 & 142 & 143 & 142 & - & 2 & 143 & 2 & 2 & 287 & 1 & 142 & 143 & 284 & 142 & - \\
\hline 287 & - & - & - & - & - & - & - & - & - & - & 7 & 41 & 4 & 7 & 166 & 1 & 40 & 52 & 143 & 91 & 7 & 41 & 4 & 7 & 166 & 1 & 40 & 52 & 80 & 143 & 63 \\
\hline 288 & 12 & 24 & 5 & 15 & 120 & 1 & 23 & 63 & 143 & 80 & 6 & 48 & 4 & 7 & 193 & 1 & 47 & 59 & 143 & 84 & 12 & 24 & 5 & 15 & 120 & 1 & 23 & 63 & 63 & 143 & 80 \\
\hline 290 & - & - & - & - & - & - & - & - & - & - & 5 & 58 & 5 & 15 & 291 & 1 & 57 & 97 & 144 & 47 & 10 & 29 & 6 & 31 & 175 & 2 & 56 & 107 & 112 & 144 & 32 \\
\hline 291 & - & - & - & - & - & - & - & - & - & - & 3 & 97 & 3 & 3 & 292 & 1 & 96 & 99 & 145 & 46 & 3 & 97 & 3 & 3 & 292 & 1 & 96 & 99 & 192 & 145 & - \\
\hline 292 & 292 & 1 & 12 & 2047 & 14 & 28 & 23 & 529 & 145 & - & 4 & 73 & 3 & 4 & 219 & 1 & 72 & 77 & 145 & 68 & 4 & 73 & 3 & 4 & 219 & 1 & 72 & 77 & 144 & 145 & 1 \\
\hline 294 & 14 & 21 & 5 & 15 & 107 & 1 & 20 & 60 & 146 & 86 & 7 & 42 & 4 & 7 & 170 & 1 & 41 & 53 & 146 & 93 & 14 & 21 & 5 & 15 & 107 & 1 & 20 & 60 & 60 & 146 & 86 \\
\hline 295 & - & - & - & - & - & - & - & - & - & - & 5 & 59 & 5 & 15 & 296 & 1 & 58 & 98 & 147 & 49 & 5 & 59 & 5 & 15 & 296 & 1 & 58 & 98 & 116 & 147 & 31 \\
\hline 296 & - & - & - & - & - & - & - & - & - & - & 8 & 37 & 5 & 15 & 185 & 1 & 36 & 76 & 147 & 71 & 8 & 37 & 5 & 15 & 185 & 1 & 36 & 76 & 76 & 147 & 71 \\
\hline 297 & - & - & - & - & - & - & - & - & - & - & 3 & 99 & 3 & 3 & 298 & 1 & 98 & 101 & 148 & 47 & 9 & 33 & 7 & 63 & 232 & 3 & 96 & 181 & 192 & 148 & - \\
\hline 298 & - & - & - & - & - & - & - & - & - & - & 2 & 149 & 2 & 2 & 299 & 1 & 148 & 149 & 148 & - & 2 & 149 & 2 & 2 & 299 & 1 & 148 & 149 & 296 & 148 & - \\
\hline 299 & - & - & - & - & - & - & - & - & - & - & - & - & - & - & - & - & - & - & - & - & - & - & - & - & - & - & - & - & - & - & - \\
\hline 300 & 15 & 20 & 5 & 15 & 102 & 1 & 19 & 59 & 149 & 90 & 15 & 20 & 5 & 15 & 102 & 2 & 38 & 55 & 149 & 94 & 30 & 10 & 6 & 31 & 62 & 3 & 27 & 58 & 58 & 149 & 91 \\
\hline 301 & - & - & - & - & - & - & - & - & - & - & 7 & 43 & 4 & 7 & 174 & 1 & 42 & 54 & 150 & 96 & 7 & 43 & 4 & 7 & 174 & 1 & 42 & 54 & 84 & 150 & 66 \\
\hline 302 & - & - & - & - & - & - & - & - & - & - & 2 & 151 & 2 & 2 & 303 & 1 & 150 & 151 & 150 & - & 2 & 151 & 2 & 2 & 303 & 1 & 150 & 151 & 300 & 150 & - \\
\hline 303 & - & - & - & - & - & - & - & - & - & - & 3 & 101 & 3 & 3 & 304 & 1 & 100 & 103 & 151 & 48 & 3 & 101 & 3 & 3 & 304 & 1 & 100 & 103 & 200 & 151 & - \\
\hline 304 & - & - & - & - & - & - & - & - & - & - & 8 & 38 & 5 & 15 & 190 & 1 & 37 & 77 & 151 & 74 & 8 & 38 & 5 & 15 & 190 & 1 & 37 & 77 & 77 & 151 & 74 \\
\hline
\end{tabular}




\begin{tabular}{|c|c|c|c|c|c|c|c|c|c|c|c|c|c|c|c|c|c|c|c|c|c|c|c|c|c|c|c|c|c|c|c|}
\hline \multirow[b]{2}{*}{$N$} & \multicolumn{10}{|c|}{ EG1 } & \multicolumn{10}{|c|}{ EG2 } & \multicolumn{11}{|c|}{ EG3 } \\
\hline & $n$ & $l$ & $m$ & $g$ & $I$ & $t$ & $F$ & $T$ & $\rho$ & $D 1$ & $n$ & $l$ & $m$ & $g$ & $I$ & $t$ & $F$ & $T$ & $\rho$ & $D 2$ & $n$ & $l$ & $m$ & $g$ & $I$ & $t$ & $F$ & $T$ & $T_{M}$ & $\rho$ & $D 3$ \\
\hline 305 & - & - & - & - & - & - & - & - & - & - & 5 & 61 & 5 & 15 & 306 & 1 & 60 & 100 & 152 & 52 & 5 & 61 & 5 & 15 & 306 & 1 & 60 & 100 & 120 & 152 & 32 \\
\hline 306 & 102 & 3 & 10 & 511 & 33 & 9 & 23 & 375 & 152 & - & 6 & 51 & 4 & 7 & 205 & 1 & 50 & 62 & 152 & 90 & 6 & 51 & 4 & 7 & 205 & 1 & 50 & 62 & 100 & 152 & 52 \\
\hline 308 & 14 & 22 & 5 & 15 & 112 & 1 & 21 & 61 & 153 & 92 & 7 & 44 & 4 & 7 & 178 & 1 & 43 & 55 & 153 & 98 & 14 & 22 & 5 & 15 & 112 & 1 & 21 & 61 & 61 & 153 & 92 \\
\hline 309 & - & - & - & - & - & - & - & - & - & - & 3 & 103 & 3 & 3 & 310 & 1 & 102 & 105 & 154 & 49 & 3 & 103 & 3 & 3 & 310 & 1 & 102 & 105 & 204 & 154 & - \\
\hline 310 & 62 & 5 & 7 & 63 & 39 & 5 & 22 & 65 & 154 & 89 & 31 & 10 & 6 & 31 & 64 & 4 & 37 & 57 & 154 & 97 & 31 & 10 & 6 & 31 & 64 & 3 & 27 & 58 & 58 & 154 & 96 \\
\hline 312 & 24 & 13 & 7 & 63 & 91 & 1 & 12 & 302 & 155 & - & 6 & 52 & 4 & 7 & 209 & 1 & 51 & 63 & 155 & 92 & 12 & 26 & 5 & 15 & 130 & 1 & 25 & 65 & 65 & 155 & 90 \\
\hline 314 & - & - & - & - & - & - & - & - & - & - & 2 & 157 & 2 & 2 & 315 & 1 & 156 & 157 & 156 & - & 2 & 157 & 2 & 2 & 315 & 1 & 156 & 157 & 312 & 156 & - \\
\hline 315 & 15 & 21 & 5 & 15 & 107 & 1 & 20 & 60 & 157 & 97 & 7 & 45 & 4 & 7 & 182 & 1 & 44 & 56 & 157 & 101 & 15 & 21 & 5 & 15 & 107 & 1 & 20 & 60 & 60 & 157 & 97 \\
\hline 316 & - & - & - & - & - & - & - & - & - & - & 4 & 79 & 3 & 4 & 237 & 1 & 78 & 83 & 157 & 74 & 4 & 79 & 3 & 4 & 237 & 1 & 78 & 83 & 156 & 157 & 1 \\
\hline 318 & - & - & - & - & - & - & - & - & - & - & 6 & 53 & 4 & 7 & 213 & 1 & 52 & 64 & 158 & 94 & 6 & 53 & 4 & 7 & 213 & 1 & 52 & 64 & 104 & 158 & 54 \\
\hline 319 & - & - & - & - & - & - & - & - & - & - & - & - & - & - & - & - & - & - & - & - & - & - & - & - & - & - & - & - & - & - & - \\
\hline 320 & 20 & 16 & 7 & 63 & 112 & 1 & 15 & 305 & 159 & - & 8 & 40 & 5 & 15 & 200 & 1 & 39 & 79 & 159 & 80 & 8 & 40 & 5 & 15 & 200 & 1 & 39 & 79 & 79 & 159 & 80 \\
\hline 321 & - & - & - & - & - & - & - & - & - & - & 3 & 107 & 3 & 3 & 322 & 1 & 106 & 109 & 160 & 51 & 3 & 107 & 3 & 3 & 322 & 1 & 106 & 109 & 212 & 160 & - \\
\hline 322 & 14 & 23 & 5 & 15 & 117 & 1 & 22 & 62 & 160 & 98 & 7 & 46 & 4 & 7 & 186 & 1 & 45 & 57 & 160 & 103 & 14 & 23 & 5 & 15 & 117 & 1 & 22 & 62 & 62 & 160 & 98 \\
\hline 323 & - & - & - & - & - & - & - & - & - & - & - & - & - & - & - & - & - & - & - & - & - & - & - & - & - & - & - & - & - & - & - \\
\hline 324 & 18 & 18 & 8 & 127 & 145 & 1 & 17 & 726 & 161 & - & 6 & 54 & 4 & 7 & 217 & 1 & 53 & 65 & 161 & 96 & 12 & 27 & 5 & 15 & 135 & 1 & 26 & 66 & 66 & 161 & 95 \\
\hline 325 & - & - & - & - & - & - & - & - & - & - & 5 & 65 & 5 & 15 & 326 & 1 & 64 & 104 & 162 & 58 & 5 & 65 & 5 & 15 & 326 & 1 & 64 & 104 & 128 & 162 & 34 \\
\hline 326 & - & - & - & - & - & - & - & - & - & - & 2 & 163 & 2 & 2 & 327 & 1 & 162 & 163 & 162 & - & 2 & 163 & 2 & 2 & 327 & 1 & 162 & 163 & 324 & 162 & - \\
\hline 327 & - & - & - & - & - & - & - & - & - & - & 3 & 109 & 3 & 3 & 328 & 1 & 108 & 111 & 163 & 52 & 3 & 109 & 3 & 3 & 328 & 1 & 108 & 111 & 216 & 163 & - \\
\hline 328 & - & - & - & - & - & - & - & - & - & - & 8 & 41 & 5 & 15 & 205 & 1 & 40 & 80 & 163 & 83 & 8 & 41 & 5 & 15 & 205 & 1 & 40 & 80 & 80 & 163 & 83 \\
\hline 329 & - & - & - & - & - & - & - & - & - & - & 7 & 47 & 4 & 7 & 190 & 1 & 46 & 58 & 164 & 106 & 7 & 47 & 4 & 7 & 190 & 1 & 46 & 58 & 92 & 164 & 72 \\
\hline 330 & 15 & 22 & 5 & 15 & 112 & 1 & 21 & 61 & 164 & 103 & 15 & 22 & 5 & 15 & 112 & 2 & 42 & 59 & 164 & 105 & 15 & 22 & 5 & 15 & 112 & 1 & 21 & 61 & 61 & 164 & 103 \\
\hline 332 & - & - & - & - & - & - & - & - & - & - & 4 & 83 & 3 & 4 & 249 & 1 & 82 & 87 & 165 & 78 & 4 & 83 & 3 & 4 & 249 & 1 & 82 & 87 & 164 & 165 & 1 \\
\hline 333 & - & - & - & - & - & - & - & - & - & - & 3 & 111 & 3 & 3 & 334 & 1 & 110 & 113 & 166 & 53 & 9 & 37 & 7 & 63 & 260 & 2 & 72 & 209 & 209 & 166 & - \\
\hline 334 & - & - & - & - & - & - & - & - & - & - & 2 & 167 & 2 & 2 & 335 & 1 & 166 & 167 & 166 & - & 2 & 167 & 2 & 2 & 335 & 1 & 166 & 167 & 332 & 166 & - \\
\hline 335 & - & - & - & - & - & - & - & - & - & - & 5 & 67 & 5 & 15 & 336 & 1 & 66 & 106 & 167 & 61 & 5 & 67 & 5 & 15 & 336 & 1 & 66 & 106 & 132 & 167 & 35 \\
\hline 336 & 14 & 24 & 5 & 15 & 122 & 1 & 23 & 63 & 167 & 104 & 7 & 48 & 4 & 7 & 194 & 1 & 47 & 59 & 167 & 108 & 14 & 24 & 5 & 15 & 122 & 1 & 23 & 63 & 63 & 167 & 104 \\
\hline 338 & - & - & - & - & - & - & - & - & - & - & 2 & 169 & 2 & 2 & 339 & 1 & 168 & 169 & 168 & - & 2 & 169 & 2 & 2 & 339 & 1 & 168 & 169 & 336 & 168 & - \\
\hline
\end{tabular}




\begin{tabular}{|c|c|c|c|c|c|c|c|c|c|c|c|c|c|c|c|c|c|c|c|c|c|c|c|c|c|c|c|c|c|c|c|}
\hline \multirow[b]{2}{*}{$N$} & \multicolumn{10}{|c|}{ EG1 } & \multicolumn{10}{|c|}{ EG2 } & \multicolumn{11}{|c|}{ EG3 } \\
\hline & $n$ & $l$ & $m$ & $g$ & $I$ & $t$ & $F$ & $T$ & $\rho$ & $D 1$ & $n$ & $l$ & $m$ & $g$ & $I$ & $t$ & $F$ & $T$ & $\rho$ & $D 2$ & $n$ & $l$ & $m$ & $g$ & $I$ & $t$ & $F$ & $T$ & $T_{M}$ & $\rho$ & D3 \\
\hline 339 & - & - & - & - & - & - & - & - & - & - & 3 & 113 & 3 & 3 & 340 & 1 & 112 & 115 & 169 & 54 & 3 & 113 & 3 & 3 & 340 & 1 & 112 & 115 & 224 & 169 & - \\
\hline 340 & 340 & 1 & 11 & 1023 & 13 & 28 & 23 & 232 & 169 & - & 4 & 85 & 3 & 4 & 255 & 1 & 84 & 89 & 169 & 80 & 170 & 2 & 10 & 511 & 24 & 34 & 62 & 125 & 125 & 169 & 44 \\
\hline 341 & 31 & 11 & 6 & 31 & 70 & 2 & 20 & 71 & 170 & 99 & 31 & 11 & 6 & 31 & 70 & 3 & 30 & 61 & 170 & 109 & 31 & 11 & 6 & 31 & 70 & 3 & 30 & 61 & 61 & 170 & 109 \\
\hline 342 & 18 & 19 & 8 & 127 & 153 & 1 & 18 & 727 & 170 & - & 6 & 57 & 4 & 7 & 229 & 1 & 56 & 68 & 170 & 102 & 6 & 57 & 4 & 7 & 229 & 1 & 56 & 68 & 112 & 170 & 58 \\
\hline 343 & - & - & - & - & - & - & - & - & - & - & 7 & 49 & 4 & 7 & 198 & 1 & 48 & 60 & 171 & 111 & 7 & 49 & 4 & 7 & 198 & 1 & 48 & 60 & 96 & 171 & 75 \\
\hline 344 & - & - & - & - & - & - & - & - & - & - & 8 & 43 & 5 & 15 & 215 & 1 & 42 & 82 & 171 & 89 & 8 & 43 & 5 & 15 & 215 & 1 & 42 & 82 & 84 & 171 & 87 \\
\hline 345 & 15 & 23 & 5 & 15 & 117 & 1 & 22 & 62 & 172 & 110 & 15 & 23 & 5 & 15 & 117 & 2 & 44 & 61 & 172 & 111 & 15 & 23 & 5 & 15 & 117 & 1 & 22 & 62 & 62 & 172 & 110 \\
\hline 346 & - & - & - & - & - & - & - & - & - & - & 2 & 173 & 2 & 2 & 347 & 1 & 172 & 173 & 172 & - & 2 & 173 & 2 & 2 & 347 & 1 & 172 & 173 & 344 & 172 & - \\
\hline 348 & - & - & - & - & - & - & - & - & - & - & 12 & 29 & 5 & 15 & 145 & 1 & 28 & 68 & 173 & 105 & 12 & 29 & 5 & 15 & 145 & 1 & 28 & 68 & 68 & 173 & 105 \\
\hline 350 & 70 & 5 & 9 & 255 & 47 & 5 & 22 & 315 & 174 & - & 7 & 50 & 4 & 7 & 202 & 1 & 49 & 61 & 174 & 113 & 14 & 25 & 5 & 15 & 127 & 1 & 24 & 64 & 64 & 174 & 110 \\
\hline 351 & - & - & - & - & - & - & - & - & - & - & 3 & 117 & 3 & 3 & 352 & 1 & 116 & 119 & 175 & 56 & 9 & 39 & 7 & 63 & 274 & 2 & 76 & 213 & 213 & 175 & - \\
\hline 352 & - & - & - & - & - & - & - & - & - & - & 8 & 44 & 5 & 15 & 220 & 1 & 43 & 83 & 175 & 92 & 8 & 44 & 5 & 15 & 220 & 1 & 43 & 83 & 86 & 175 & 89 \\
\hline 354 & - & - & - & - & - & - & - & - & - & - & 6 & 59 & 4 & 7 & 237 & 1 & 58 & 70 & 176 & 106 & 6 & 59 & 4 & 7 & 237 & 1 & 58 & 70 & 116 & 176 & 60 \\
\hline 355 & - & - & - & - & - & - & - & - & - & - & 5 & 71 & 5 & 15 & 356 & 1 & 70 & 110 & 177 & 67 & 5 & 71 & 5 & 15 & 356 & 1 & 70 & 110 & 140 & 177 & 37 \\
\hline 356 & - & - & - & - & - & - & - & - & - & - & 4 & 89 & 3 & 4 & 267 & 1 & 88 & 93 & 177 & 84 & 4 & 89 & 3 & 4 & 267 & 1 & 88 & 93 & 176 & 177 & 1 \\
\hline 357 & 21 & 17 & 6 & 31 & 104 & 1 & 16 & 129 & 178 & 49 & 7 & 51 & 4 & 7 & 206 & 1 & 50 & 62 & 178 & 116 & 21 & 17 & 6 & 31 & 104 & 2 & 32 & 83 & 83 & 178 & 95 \\
\hline 358 & - & - & - & - & - & - & - & - & - & - & 2 & 179 & 2 & 2 & 359 & 1 & 178 & 179 & 178 & - & 2 & 179 & 2 & 2 & 359 & 1 & 178 & 179 & 356 & 178 & - \\
\hline 360 & 15 & 24 & 5 & 15 & 122 & 1 & 23 & 63 & 179 & 116 & 15 & 24 & 5 & 15 & 122 & 2 & 46 & 63 & 179 & 116 & 15 & 24 & 5 & 15 & 122 & 1 & 23 & 63 & 63 & 179 & 116 \\
\hline 361 & - & - & - & - & - & - & - & - & - & - & - & - & - & - & - & - & - & - & - & - & - & - & - & - & - & - & - & - & - & - & - \\
\hline 362 & - & - & - & - & - & - & - & - & - & - & 2 & 181 & 2 & 2 & 363 & 1 & 180 & 181 & 180 & - & 2 & 181 & 2 & 2 & 363 & 1 & 180 & 181 & 360 & 180 & - \\
\hline 363 & - & - & - & - & - & - & - & - & - & - & 3 & 121 & 3 & 3 & 364 & 1 & 120 & 123 & 181 & 58 & 3 & 121 & 3 & 3 & 364 & 1 & 120 & 123 & 240 & 181 & - \\
\hline 364 & 28 & 13 & 6 & 31 & 79 & 1 & 12 & 125 & 181 & 56 & 7 & 52 & 4 & 7 & 210 & 1 & 51 & 63 & 181 & 118 & 14 & 26 & 5 & 15 & 132 & 1 & 25 & 65 & 65 & 181 & 116 \\
\hline 365 & 73 & 5 & 10 & 511 & 54 & 5 & 22 & 724 & 182 & - & 5 & 73 & 5 & 15 & 366 & 1 & 72 & 112 & 182 & 70 & 5 & 73 & 5 & 15 & 366 & 1 & 72 & 112 & 144 & 182 & 38 \\
\hline 366 & - & - & - & - & - & - & - & - & - & - & 6 & 61 & 4 & 7 & 245 & 1 & 60 & 72 & 182 & 110 & 6 & 61 & 4 & 7 & 245 & 1 & 60 & 72 & 120 & 182 & 62 \\
\hline 368 & - & - & - & - & - & - & - & - & - & - & 8 & 46 & 5 & 15 & 230 & 1 & 45 & 85 & 183 & 98 & 8 & 46 & 5 & 15 & 230 & 1 & 45 & 85 & 90 & 183 & 93 \\
\hline 369 & - & - & - & - & - & - & - & - & - & - & 3 & 123 & 3 & 3 & 370 & 1 & 122 & 125 & 184 & 59 & 9 & 41 & 7 & 63 & 288 & 2 & 80 & 217 & 217 & 184 & - \\
\hline 370 & - & - & - & - & - & - & - & - & - & - & 5 & 74 & 5 & 15 & 371 & 1 & 73 & 113 & 184 & 71 & 10 & 37 & 6 & 31 & 223 & 2 & 72 & 123 & 144 & 184 & 40 \\
\hline 371 & - & - & - & - & - & - & - & - & - & - & 7 & 53 & 4 & 7 & 214 & 1 & 52 & 64 & 185 & 121 & 7 & 53 & 4 & 7 & 214 & 1 & 52 & 64 & 104 & 185 & 81 \\
\hline
\end{tabular}




\begin{tabular}{|c|c|c|c|c|c|c|c|c|c|c|c|c|c|c|c|c|c|c|c|c|c|c|c|c|c|c|c|c|c|c|c|}
\hline \multirow[b]{2}{*}{$N$} & \multicolumn{10}{|c|}{ EG1 } & \multicolumn{10}{|c|}{ EG2 } & \multicolumn{11}{|c|}{ EG3 } \\
\hline & $n$ & $l$ & $m$ & $g$ & $I$ & $t$ & $F$ & $T$ & $\rho$ & $D 1$ & $n$ & $l$ & $m$ & $g$ & $I$ & $t$ & $F$ & $T$ & $\rho$ & $D 2$ & $n$ & $l$ & $m$ & $g$ & $I$ & $t$ & $F$ & $T$ & $T_{M}$ & $\rho$ & D3 \\
\hline 372 & 31 & 12 & 6 & 31 & 76 & 2 & 22 & 73 & 185 & 112 & 31 & 12 & 6 & 31 & 76 & 3 & 33 & 64 & 185 & 121 & 31 & 12 & 6 & 31 & 76 & 3 & 33 & 64 & 66 & 185 & 119 \\
\hline 374 & - & - & - & - & - & - & - & - & - & - & 2 & 187 & 2 & 2 & 375 & 1 & 186 & 187 & 186 & - & 2 & 187 & 2 & 2 & 375 & 1 & 186 & 187 & 372 & 186 & - \\
\hline 375 & - & - & - & - & - & - & - & - & - & - & 15 & 25 & 5 & 15 & 127 & 1 & 24 & 64 & 187 & 123 & 15 & 25 & 5 & 15 & 127 & 1 & 24 & 64 & 64 & 187 & 123 \\
\hline 376 & - & - & - & - & - & - & - & - & - & - & 8 & 47 & 5 & 15 & 235 & 1 & 46 & 86 & 187 & 101 & 8 & 47 & 5 & 15 & 235 & 1 & 46 & 86 & 92 & 187 & 95 \\
\hline 377 & - & - & - & - & - & - & - & - & - & - & - & - & - & - & - & - & - & - & - & - & - & - & - & - & - & - & - & - & - & - & - \\
\hline 378 & 126 & 3 & 8 & 127 & 27 & 9 & 23 & 77 & 188 & 111 & 7 & 54 & 4 & 7 & 218 & 1 & 53 & 65 & 188 & 123 & 14 & 27 & 5 & 15 & 137 & 1 & 26 & 66 & 66 & 188 & 122 \\
\hline 380 & 20 & 19 & 7 & 63 & 133 & 1 & 18 & 308 & 189 & - & 4 & 95 & 3 & 4 & 285 & 1 & 94 & 99 & 189 & 90 & 20 & 19 & 7 & 63 & 133 & 3 & 54 & 139 & 139 & 189 & 50 \\
\hline 381 & 127 & 3 & 8 & 127 & 29 & 9 & 23 & 77 & 190 & 113 & 127 & 3 & 8 & 127 & 29 & 14 & 37 & 66 & 190 & 124 & 127 & 3 & 8 & 127 & 29 & 12 & 32 & 68 & 68 & 190 & 122 \\
\hline 382 & - & - & - & - & - & - & - & - & - & - & 2 & 191 & 2 & 2 & 383 & 1 & 190 & 191 & 190 & - & 2 & 191 & 2 & 2 & 383 & 1 & 190 & 191 & 380 & 190 & - \\
\hline 384 & 24 & 16 & 7 & 63 & 112 & 1 & 15 & 305 & 191 & - & 12 & 32 & 5 & 15 & 160 & 1 & 31 & 71 & 191 & 120 & 12 & 32 & 5 & 15 & 160 & 1 & 31 & 71 & 71 & 191 & 120 \\
\hline 385 & 35 & 11 & 8 & 127 & 90 & 2 & 20 & 362 & 192 & - & 7 & 55 & 4 & 7 & 222 & 1 & 54 & 66 & 192 & 126 & 7 & 55 & 4 & 7 & 222 & 1 & 54 & 66 & 108 & 192 & 84 \\
\hline 386 & - & - & - & - & - & - & - & - & - & - & 2 & 193 & 2 & 2 & 387 & 1 & 192 & 193 & 192 & - & 2 & 193 & 2 & 2 & 387 & 1 & 192 & 193 & 384 & 192 & - \\
\hline 387 & - & - & - & - & - & - & - & - & - & - & 3 & 129 & 3 & 3 & 388 & 1 & 128 & 131 & 193 & 62 & 9 & 43 & 7 & 63 & 302 & 2 & 84 & 221 & 221 & 193 & - \\
\hline 388 & - & - & - & - & - & - & - & - & - & - & 4 & 97 & 3 & 4 & 291 & 1 & 96 & 101 & 193 & 92 & 4 & 97 & 3 & 4 & 291 & 1 & 96 & 101 & 192 & 193 & 1 \\
\hline 390 & 30 & 13 & 6 & 31 & 80 & 1 & 12 & 125 & 194 & 69 & 15 & 26 & 5 & 15 & 132 & 1 & 25 & 65 & 194 & 129 & 15 & 26 & 5 & 15 & 132 & 1 & 25 & 65 & 65 & 194 & 129 \\
\hline 391 & - & - & - & - & - & - & - & - & - & - & - & - & - & - & - & - & - & - & - & - & - & - & - & - & - & - & - & - & - & - & - \\
\hline 392 & 28 & 14 & 6 & 31 & 85 & 1 & 13 & 126 & 195 & 69 & 7 & 56 & 4 & 7 & 226 & 1 & 55 & 67 & 195 & 128 & 14 & 28 & 5 & 15 & 142 & 1 & 27 & 67 & 67 & 195 & 128 \\
\hline 393 & - & - & - & - & - & - & - & - & - & - & 3 & 131 & 3 & 3 & 394 & 1 & 130 & 133 & 196 & 63 & 3 & 131 & 3 & 3 & 394 & 1 & 130 & 133 & 260 & 196 & - \\
\hline 394 & - & - & - & - & - & - & - & - & - & - & 2 & 197 & 2 & 2 & 395 & 1 & 196 & 197 & 196 & - & 2 & 197 & 2 & 2 & 395 & 1 & 196 & 197 & 392 & 196 & - \\
\hline 395 & - & - & - & - & - & - & - & - & - & - & 5 & 79 & 5 & 15 & 396 & 1 & 78 & 118 & 197 & 79 & 5 & 79 & 5 & 15 & 396 & 1 & 78 & 118 & 156 & 197 & 41 \\
\hline 396 & 18 & 22 & 8 & 127 & 177 & 1 & 21 & 730 & 197 & - & 12 & 33 & 5 & 15 & 165 & 1 & 32 & 72 & 197 & 125 & 12 & 33 & 5 & 15 & 165 & 1 & 32 & 72 & 72 & 197 & 125 \\
\hline 398 & - & - & - & - & - & - & - & - & - & - & 2 & 199 & 2 & 2 & 399 & 1 & 198 & 199 & 198 & - & 2 & 199 & 2 & 2 & 399 & 1 & 198 & 199 & 396 & 198 & - \\
\hline 399 & 21 & 19 & 6 & 31 & 116 & 1 & 18 & 131 & 199 & 68 & 7 & 57 & 4 & 7 & 230 & 1 & 56 & 68 & 199 & 131 & 21 & 19 & 6 & 31 & 116 & 2 & 36 & 87 & 87 & 199 & 112 \\
\hline 400 & 20 & 20 & 7 & 63 & 140 & 1 & 19 & 309 & 199 & - & 8 & 50 & 5 & 15 & 250 & 1 & 49 & 89 & 199 & 110 & 8 & 50 & 5 & 15 & 250 & 1 & 49 & 89 & 98 & 199 & 101 \\
\hline 402 & - & - & - & - & - & - & - & - & - & - & 6 & 67 & 4 & 7 & 269 & 1 & 66 & 78 & 200 & 122 & 6 & 67 & 4 & 7 & 269 & 1 & 66 & 78 & 132 & 200 & 68 \\
\hline 403 & 31 & 13 & 6 & 31 & 82 & 1 & 12 & 125 & 201 & 76 & 31 & 13 & 6 & 31 & 82 & 3 & 36 & 67 & 201 & 134 & 31 & 13 & 6 & 31 & 82 & 3 & 36 & 67 & 72 & 201 & 129 \\
\hline 404 & - & - & - & - & - & - & - & - & - & - & 4 & 101 & 3 & 4 & 303 & 1 & 100 & 105 & 201 & 96 & 4 & 101 & 3 & 4 & 303 & 1 & 100 & 105 & 200 & 201 & 1 \\
\hline 405 & - & - & - & - & - & - & - & - & - & - & 15 & 27 & 5 & 15 & 137 & 1 & 26 & 66 & 202 & 136 & 15 & 27 & 5 & 15 & 137 & 1 & 26 & 66 & 66 & 202 & 136 \\
\hline
\end{tabular}




\begin{tabular}{|c|c|c|c|c|c|c|c|c|c|c|c|c|c|c|c|c|c|c|c|c|c|c|c|c|c|c|c|c|c|c|c|}
\hline \multirow[b]{2}{*}{$N$} & \multicolumn{10}{|c|}{ EG1 } & \multicolumn{10}{|c|}{ EG2 } & \multicolumn{11}{|c|}{ EG3 } \\
\hline & $n$ & $l$ & $m$ & $g$ & $I$ & $t$ & $F$ & $T$ & $\rho$ & $D 1$ & $n$ & $l$ & $m$ & $g$ & $I$ & $t$ & $F$ & $T$ & $\rho$ & $D 2$ & $n$ & $l$ & $m$ & $g$ & $I$ & $t$ & $F$ & $T$ & $T_{M}$ & $\rho$ & $D 3$ \\
\hline 406 & - & - & - & - & - & - & - & - & - & - & 14 & 29 & 5 & 15 & 147 & 1 & 28 & 68 & 202 & 134 & 14 & 29 & 5 & 15 & 147 & 1 & 28 & 68 & 68 & 202 & 134 \\
\hline 407 & - & - & - & - & - & - & - & - & - & - & - & - & - & - & - & - & - & - & - & - & - & - & - & - & - & - & - & - & - & - & - \\
\hline 408 & 24 & 17 & 7 & 63 & 119 & 1 & 16 & 306 & 203 & - & 12 & 34 & 5 & 15 & 170 & 1 & 33 & 73 & 203 & 130 & 12 & 34 & 5 & 15 & 170 & 1 & 33 & 73 & 73 & 203 & 130 \\
\hline 410 & - & - & - & - & - & - & - & - & - & - & 5 & 82 & 5 & 15 & 411 & 1 & 81 & 121 & 204 & 83 & 10 & 41 & 6 & 31 & 247 & 1 & 40 & 153 & 153 & 204 & 51 \\
\hline 411 & - & - & - & - & - & - & - & - & - & - & 3 & 137 & 3 & 3 & 412 & 1 & 136 & 139 & 205 & 66 & 3 & 137 & 3 & 3 & 412 & 1 & 136 & 139 & 272 & 205 & - \\
\hline 412 & - & - & - & - & - & - & - & - & - & - & 4 & 103 & 3 & 4 & 309 & 1 & 102 & 107 & 205 & 98 & 4 & 103 & 3 & 4 & 309 & 1 & 102 & 107 & 204 & 205 & 1 \\
\hline 413 & - & - & - & - & - & - & - & - & - & - & 7 & 59 & 4 & 7 & 238 & 1 & 58 & 70 & 206 & 136 & 7 & 59 & 4 & 7 & 238 & 1 & 58 & 70 & 116 & 206 & 90 \\
\hline 414 & 18 & 23 & 8 & 127 & 185 & 1 & 22 & 731 & 206 & - & 6 & 69 & 4 & 7 & 277 & 1 & 68 & 80 & 206 & 126 & 6 & 69 & 4 & 7 & 277 & 1 & 68 & 80 & 136 & 206 & 70 \\
\hline 415 & - & - & - & - & - & - & - & - & - & - & 5 & 83 & 5 & 15 & 416 & 1 & 82 & 122 & 207 & 85 & 5 & 83 & 5 & 15 & 416 & 1 & 82 & 122 & 164 & 207 & 43 \\
\hline 416 & - & - & - & - & - & - & - & - & - & - & 8 & 52 & 5 & 15 & 260 & 1 & 51 & 91 & 207 & 116 & 8 & 52 & 5 & 15 & 260 & 1 & 51 & 91 & 102 & 207 & 105 \\
\hline 417 & - & - & - & - & - & - & - & - & - & - & 3 & 139 & 3 & 3 & 418 & 1 & 138 & 141 & 208 & 67 & 3 & 139 & 3 & 3 & 418 & 1 & 138 & 141 & 276 & 208 & - \\
\hline 418 & - & - & - & - & - & - & - & - & - & - & 2 & 209 & 2 & 2 & 419 & 1 & 208 & 209 & 208 & - & 2 & 209 & 2 & 2 & 419 & 1 & 208 & 209 & 416 & 208 & - \\
\hline 420 & 60 & 7 & 7 & 63 & 50 & 3 & 18 & 103 & 209 & 106 & 15 & 28 & 5 & 15 & 142 & 1 & 27 & 67 & 209 & 142 & 15 & 28 & 5 & 15 & 142 & 1 & 27 & 67 & 67 & 209 & 142 \\
\hline 422 & - & - & - & - & - & - & - & - & - & - & 2 & 211 & 2 & 2 & 423 & 1 & 210 & 211 & 210 & - & 2 & 211 & 2 & 2 & 423 & 1 & 210 & 211 & 420 & 210 & - \\
\hline 423 & - & - & - & - & - & - & - & - & - & - & 3 & 141 & 3 & 3 & 424 & 1 & 140 & 143 & 211 & 68 & 9 & 47 & 7 & 63 & 330 & 2 & 92 & 229 & 229 & 211 & - \\
\hline 424 & - & - & - & - & - & - & - & - & - & - & 8 & 53 & 5 & 15 & 265 & 1 & 52 & 92 & 211 & 119 & 8 & 53 & 5 & 15 & 265 & 1 & 52 & 92 & 104 & 211 & 107 \\
\hline 425 & 85 & 5 & 9 & 255 & 49 & 5 & 22 & 315 & 212 & - & 5 & 85 & 5 & 15 & 426 & 1 & 84 & 124 & 212 & 88 & 85 & 5 & 9 & 255 & 49 & 15 & 70 & 143 & 143 & 212 & 69 \\
\hline 426 & - & - & - & - & - & - & - & - & - & - & 6 & 71 & 4 & 7 & 285 & 1 & 70 & 82 & 212 & 130 & 6 & 71 & 4 & 7 & 285 & 1 & 70 & 82 & 140 & 212 & 72 \\
\hline 427 & - & - & - & - & - & - & - & - & - & - & 7 & 61 & 4 & 7 & 246 & 1 & 60 & 72 & 213 & 141 & 7 & 61 & 4 & 7 & 246 & 1 & 60 & 72 & 120 & 213 & 93 \\
\hline 428 & - & - & - & - & - & - & - & - & - & - & 4 & 107 & 3 & 4 & 321 & 1 & 106 & 111 & 213 & 102 & 4 & 107 & 3 & 4 & 321 & 1 & 106 & 111 & 212 & 213 & 1 \\
\hline 429 & - & - & - & - & - & - & - & - & - & - & 3 & 143 & 3 & 3 & 430 & 1 & 142 & 145 & 214 & 69 & 3 & 143 & 3 & 3 & 430 & 1 & 142 & 145 & 284 & 214 & - \\
\hline 430 & - & - & - & - & - & - & - & - & - & - & 5 & 86 & 5 & 15 & 431 & 1 & 85 & 125 & 214 & 89 & 10 & 43 & 6 & 31 & 259 & 1 & 42 & 155 & 155 & 214 & 59 \\
\hline 432 & 24 & 18 & 7 & 63 & 126 & 1 & 17 & 307 & 215 & - & 12 & 36 & 5 & 15 & 180 & 1 & 35 & 75 & 215 & 140 & 12 & 36 & 5 & 15 & 180 & 1 & 35 & 75 & 75 & 215 & 140 \\
\hline 434 & 62 & 7 & 7 & 63 & 53 & 3 & 18 & 103 & 216 & 113 & 14 & 31 & 5 & 15 & 157 & 1 & 30 & 70 & 216 & 146 & 14 & 31 & 5 & 15 & 157 & 1 & 30 & 70 & 70 & 216 & 146 \\
\hline 435 & - & - & - & - & - & - & - & - & - & - & 15 & 29 & 5 & 15 & 147 & 1 & 28 & 68 & 217 & 149 & 15 & 29 & 5 & 15 & 147 & 1 & 28 & 68 & 68 & 217 & 149 \\
\hline 436 & - & - & - & - & - & - & - & - & - & - & 4 & 109 & 3 & 4 & 327 & 1 & 108 & 113 & 217 & 104 & 4 & 109 & 3 & 4 & 327 & 1 & 108 & 113 & 216 & 217 & 1 \\
\hline 437 & - & - & - & - & - & - & - & - & - & - & - & - & - & - & - & - & - & - & - & - & - & - & - & - & - & - & - & - & - & - & - \\
\hline 438 & 146 & 3 & 11 & 1023 & 37 & 9 & 23 & 859 & 218 & - & 6 & 73 & 4 & 7 & 293 & 1 & 72 & 84 & 218 & 134 & 6 & 73 & 4 & 7 & 293 & 1 & 72 & 84 & 144 & 218 & 74 \\
\hline
\end{tabular}




\begin{tabular}{|c|c|c|c|c|c|c|c|c|c|c|c|c|c|c|c|c|c|c|c|c|c|c|c|c|c|c|c|c|c|c|c|}
\hline \multirow[b]{2}{*}{$N$} & \multicolumn{10}{|c|}{ EG1 } & \multicolumn{10}{|c|}{ EG2 } & \multicolumn{11}{|c|}{ EG3 } \\
\hline & $n$ & $l$ & $m$ & $g$ & $I$ & $t$ & $F$ & $T$ & $\rho$ & $D 1$ & $n$ & $l$ & $m$ & $g$ & $I$ & $t$ & $F$ & $T$ & $\rho$ & $D 2$ & $n$ & $l$ & $m$ & $g$ & $I$ & $t$ & $F$ & $T$ & $T_{M}$ & $\rho$ & $D 3$ \\
\hline 440 & 20 & 22 & 7 & 63 & 154 & 1 & 21 & 311 & 219 & - & 8 & 55 & 5 & 15 & 275 & 1 & 54 & 94 & 219 & 125 & 8 & 55 & 5 & 15 & 275 & 1 & 54 & 94 & 108 & 219 & 111 \\
\hline 441 & 63 & 7 & 7 & 63 & 53 & 3 & 18 & 103 & 220 & 117 & 63 & 7 & 7 & 63 & 53 & 6 & 38 & 72 & 220 & 148 & 63 & 7 & 7 & 63 & 53 & 5 & 32 & 75 & 75 & 220 & 145 \\
\hline 442 & - & - & - & - & - & - & - & - & - & - & 2 & 221 & 2 & 2 & 443 & 1 & 220 & 221 & 220 & - & 2 & 221 & 2 & 2 & 443 & 1 & 220 & 221 & 440 & 220 & - \\
\hline 444 & - & - & - & - & - & - & - & - & - & - & 12 & 37 & 5 & 15 & 185 & 1 & 36 & 76 & 221 & 145 & 12 & 37 & 5 & 15 & 185 & 1 & 36 & 76 & 76 & 221 & 145 \\
\hline 445 & - & - & - & - & - & - & - & - & - & - & 5 & 89 & 5 & 15 & 446 & 1 & 88 & 128 & 222 & 94 & 5 & 89 & 5 & 15 & 446 & 1 & 88 & 128 & 176 & 222 & 46 \\
\hline 446 & - & - & - & - & - & - & - & - & - & - & 2 & 223 & 2 & 2 & 447 & 1 & 222 & 223 & 222 & - & 2 & 223 & 2 & 2 & 447 & 1 & 222 & 223 & 444 & 222 & - \\
\hline 447 & - & - & - & - & - & - & - & - & - & - & 3 & 149 & 3 & 3 & 448 & 1 & 148 & 151 & 223 & 72 & 3 & 149 & 3 & 3 & 448 & 1 & 148 & 151 & 296 & 223 & - \\
\hline 448 & 28 & 16 & 6 & 31 & 97 & 1 & 15 & 128 & 223 & 95 & 14 & 32 & 5 & 15 & 162 & 1 & 31 & 71 & 223 & 152 & 14 & 32 & 5 & 15 & 162 & 1 & 31 & 71 & 71 & 223 & 152 \\
\hline 450 & 30 & 15 & 6 & 31 & 92 & 1 & 14 & 127 & 224 & 97 & 15 & 30 & 5 & 15 & 152 & 1 & 29 & 69 & 224 & 155 & 15 & 30 & 5 & 15 & 152 & 1 & 29 & 69 & 69 & 224 & 155 \\
\hline 451 & - & - & - & - & - & - & - & - & - & - & - & - & - & - & - & - & - & - & - & - & - & - & - & - & - & - & - & - & - & - & - \\
\hline 452 & - & - & - & - & - & - & - & - & - & - & 4 & 113 & 3 & 4 & 339 & 1 & 112 & 117 & 225 & 108 & 4 & 113 & 3 & 4 & 339 & 1 & 112 & 117 & 224 & 225 & 1 \\
\hline 453 & - & - & - & - & - & - & - & - & - & - & 3 & 151 & 3 & 3 & 454 & 1 & 150 & 153 & 226 & 73 & 3 & 151 & 3 & 3 & 454 & 1 & 150 & 153 & 300 & 226 & - \\
\hline 454 & - & - & - & - & - & - & - & - & - & - & 2 & 227 & 2 & 2 & 455 & 1 & 226 & 227 & 226 & - & 2 & 227 & 2 & 2 & 455 & 1 & 226 & 227 & 452 & 226 & - \\
\hline 455 & 35 & 13 & 8 & 127 & 106 & 1 & 12 & 721 & 227 & - & 7 & 65 & 4 & 7 & 262 & 1 & 64 & 76 & 227 & 151 & 7 & 65 & 4 & 7 & 262 & 1 & 64 & 76 & 128 & 227 & 99 \\
\hline 456 & 24 & 19 & 7 & 63 & 133 & 1 & 18 & 308 & 227 & - & 12 & 38 & 5 & 15 & 190 & 1 & 37 & 77 & 227 & 150 & 12 & 38 & 5 & 15 & 190 & 1 & 37 & 77 & 77 & 227 & 150 \\
\hline 458 & - & - & - & - & - & - & - & - & - & - & 2 & 229 & 2 & 2 & 459 & 1 & 228 & 229 & 228 & - & 2 & 229 & 2 & 2 & 459 & 1 & 228 & 229 & 456 & 228 & - \\
\hline 459 & 51 & 9 & 9 & 255 & 84 & 2 & 16 & 835 & 229 & - & 3 & 153 & 3 & 3 & 460 & 1 & 152 & 155 & 229 & 74 & 51 & 9 & 9 & 255 & 84 & 11 & 95 & 204 & 204 & 229 & 25 \\
\hline 460 & 20 & 23 & 7 & 63 & 161 & 1 & 22 & 312 & 229 & - & 4 & 115 & 3 & 4 & 345 & 1 & 114 & 119 & 229 & 110 & 20 & 23 & 7 & 63 & 161 & 3 & 66 & 151 & 151 & 229 & 78 \\
\hline 462 & 21 & 22 & 6 & 31 & 134 & 1 & 21 & 134 & 230 & 96 & 14 & 33 & 5 & 15 & 167 & 1 & 32 & 72 & 230 & 158 & 14 & 33 & 5 & 15 & 167 & 1 & 32 & 72 & 72 & 230 & 158 \\
\hline 464 & - & - & - & - & - & - & - & - & - & - & 8 & 58 & 5 & 15 & 290 & 1 & 57 & 97 & 231 & 134 & 8 & 58 & 5 & 15 & 290 & 1 & 57 & 97 & 114 & 231 & 117 \\
\hline 465 & 465 & 1 & 10 & 511 & 14 & 28 & 23 & 105 & 232 & 127 & 15 & 31 & 5 & 15 & 157 & 1 & 30 & 70 & 232 & 162 & 15 & 31 & 5 & 15 & 157 & 1 & 30 & 70 & 70 & 232 & 162 \\
\hline 466 & - & - & - & - & - & - & - & - & - & - & 2 & 233 & 2 & 2 & 467 & 1 & 232 & 233 & 232 & - & 2 & 233 & 2 & 2 & 467 & 1 & 232 & 233 & 464 & 232 & - \\
\hline 468 & 36 & 13 & 9 & 255 & 117 & 1 & 12 & 1688 & 233 & - & 12 & 39 & 5 & 15 & 195 & 1 & 38 & 78 & 233 & 155 & 12 & 39 & 5 & 15 & 195 & 1 & 38 & 78 & 78 & 233 & 155 \\
\hline 469 & - & - & - & - & - & - & - & - & - & - & 7 & 67 & 4 & 7 & 270 & 1 & 66 & 78 & 234 & 156 & 7 & 67 & 4 & 7 & 270 & 1 & 66 & 78 & 132 & 234 & 102 \\
\hline 470 & - & - & - & - & - & - & - & - & - & - & 5 & 94 & 5 & 15 & 471 & 1 & 93 & 133 & 234 & 101 & 10 & 47 & 6 & 31 & 283 & 1 & 46 & 159 & 159 & 234 & 75 \\
\hline 471 & - & - & - & - & - & - & - & - & - & - & 3 & 157 & 3 & 3 & 472 & 1 & 156 & 159 & 235 & 76 & 3 & 157 & 3 & 3 & 472 & 1 & 156 & 159 & 312 & 235 & - \\
\hline 472 & - & - & - & - & - & - & - & - & - & - & 8 & 59 & 5 & 15 & 295 & 1 & 58 & 98 & 235 & 137 & 8 & 59 & 5 & 15 & 295 & 1 & 58 & 98 & 116 & 235 & 119 \\
\hline 473 & - & - & - & - & - & - & - & - & - & - & - & - & - & - & - & - & - & - & - & - & - & - & - & - & - & - & - & - & - & - & - \\
\hline
\end{tabular}




\begin{tabular}{|c|c|c|c|c|c|c|c|c|c|c|c|c|c|c|c|c|c|c|c|c|c|c|c|c|c|c|c|c|c|c|c|}
\hline \multirow[b]{2}{*}{$N$} & \multicolumn{10}{|c|}{ EG1 } & \multicolumn{10}{|c|}{ EG2 } & \multicolumn{11}{|c|}{ EG3 } \\
\hline & $n$ & $l$ & $m$ & $g$ & $I$ & $t$ & $F$ & $T$ & $\rho$ & $D 1$ & $n$ & $l$ & $m$ & $g$ & $I$ & $t$ & $F$ & $T$ & $\rho$ & D2 & $n$ & $l$ & $m$ & $g$ & $I$ & $t$ & $F$ & $T$ & $T_{M}$ & $\rho$ & D3 \\
\hline 474 & - & - & - & - & - & - & - & - & - & - & 6 & 79 & 4 & 7 & 317 & 1 & 78 & 90 & 236 & 146 & 6 & 79 & 4 & 7 & 317 & 1 & 78 & 90 & 156 & 236 & 80 \\
\hline 475 & - & - & - & - & - & - & - & - & - & - & 5 & 95 & 5 & 15 & 476 & 1 & 94 & 134 & 237 & 103 & 5 & 95 & 5 & 15 & 476 & 1 & 94 & 134 & 188 & 237 & 49 \\
\hline 476 & 28 & 17 & 6 & 31 & 103 & 1 & 16 & 129 & 237 & 108 & 14 & 34 & 5 & 15 & 172 & 1 & 33 & 73 & 237 & 164 & 14 & 34 & 5 & 15 & 172 & 1 & 33 & 73 & 73 & 237 & 164 \\
\hline 477 & - & - & - & - & - & - & - & - & - & - & 3 & 159 & 3 & 3 & 478 & 1 & 158 & 161 & 238 & 77 & 9 & 53 & 7 & 63 & 372 & 2 & 104 & 241 & 241 & 238 & - \\
\hline 478 & - & - & - & - & - & - & - & - & - & - & 2 & 239 & 2 & 2 & 479 & 1 & 238 & 239 & 238 & - & 2 & 239 & 2 & 2 & 479 & 1 & 238 & 239 & 476 & 238 & - \\
\hline 480 & 60 & 8 & 7 & 63 & 57 & 3 & 21 & 106 & 239 & 133 & 15 & 32 & 5 & 15 & 162 & 1 & 31 & 71 & 239 & 168 & 15 & 32 & 5 & 15 & 162 & 1 & 31 & 71 & 71 & 239 & 168 \\
\hline 481 & - & - & - & - & - & - & - & - & - & - & - & - & - & - & - & - & - & - & - & - & - & - & - & - & - & - & - & - & - & - & - \\
\hline 482 & - & - & - & - & - & - & - & - & - & - & 2 & 241 & 2 & 2 & 483 & 1 & 240 & 241 & 240 & - & 2 & 241 & 2 & 2 & 483 & 1 & 240 & 241 & 480 & 240 & - \\
\hline 483 & 21 & 23 & 6 & 31 & 140 & 1 & 22 & 135 & 241 & 106 & 7 & 69 & 4 & 7 & 278 & 1 & 68 & 80 & 241 & 161 & 21 & 23 & 6 & 31 & 140 & 2 & 44 & 95 & 95 & 241 & 146 \\
\hline 484 & - & - & - & - & - & - & - & - & - & - & 4 & 121 & 3 & 4 & 363 & 1 & 120 & 125 & 241 & 116 & 4 & 121 & 3 & 4 & 363 & 1 & 120 & 125 & 240 & 241 & 1 \\
\hline 485 & - & - & - & - & - & - & - & - & - & - & 5 & 97 & 5 & 15 & 486 & 1 & 96 & 136 & 242 & 106 & 5 & 97 & 5 & 15 & 486 & 1 & 96 & 136 & 192 & 242 & 50 \\
\hline 486 & - & - & - & - & - & - & - & - & - & - & 6 & 81 & 4 & 7 & 325 & 1 & 80 & 92 & 242 & 150 & 6 & 81 & 4 & 7 & 325 & 1 & 80 & 92 & 160 & 242 & 82 \\
\hline 488 & - & - & - & - & - & - & - & - & - & - & 8 & 61 & 5 & 15 & 305 & 1 & 60 & 100 & 243 & 143 & 8 & 61 & 5 & 15 & 305 & 1 & 60 & 100 & 120 & 243 & 123 \\
\hline 490 & 70 & 7 & 9 & 255 & 65 & 3 & 18 & 545 & 244 & - & 14 & 35 & 5 & 15 & 177 & 1 & 34 & 74 & 244 & 170 & 14 & 35 & 5 & 15 & 177 & 1 & 34 & 74 & 74 & 244 & 170 \\
\hline 492 & - & - & - & - & - & - & - & - & - & - & 12 & 41 & 5 & 15 & 205 & 1 & 40 & 80 & 245 & 165 & 12 & 41 & 5 & 15 & 205 & 1 & 40 & 80 & 80 & 245 & 165 \\
\hline 493 & - & - & - & - & - & - & - & - & - & - & - & - & - & - & - & - & - & - & - & - & - & - & - & - & - & - & - & - & - & - & - \\
\hline 494 & - & - & - & - & - & - & - & - & - & - & 2 & 247 & 2 & 2 & 495 & 1 & 246 & 247 & 246 & - & 2 & 247 & 2 & 2 & 495 & 1 & 246 & 247 & 492 & 246 & - \\
\hline 495 & - & - & - & - & - & - & - & - & - & - & 15 & 33 & 5 & 15 & 167 & 1 & 32 & 72 & 247 & 175 & 15 & 33 & 5 & 15 & 167 & 1 & 32 & 72 & 72 & 247 & 175 \\
\hline 496 & 62 & 8 & 7 & 63 & 60 & 3 & 21 & 106 & 247 & 141 & 31 & 16 & 6 & 31 & 100 & 3 & 45 & 76 & 247 & 171 & 62 & 8 & 7 & 63 & 60 & 5 & 37 & 80 & 80 & 247 & 167 \\
\hline 497 & - & - & - & - & - & - & - & - & - & - & 7 & 71 & 4 & 7 & 286 & 1 & 70 & 82 & 248 & 166 & 7 & 71 & 4 & 7 & 286 & 1 & 70 & 82 & 140 & 248 & 108 \\
\hline 498 & - & - & - & - & - & - & - & - & - & - & 6 & 83 & 4 & 7 & 333 & 1 & 82 & 94 & 248 & 154 & 6 & 83 & 4 & 7 & 333 & 1 & 82 & 94 & 164 & 248 & 84 \\
\hline 500 & - & - & - & - & - & - & - & - & - & - & 4 & 125 & 3 & 4 & 375 & 1 & 124 & 129 & 249 & 120 & 20 & 25 & 7 & 63 & 175 & 3 & 72 & 157 & 157 & 249 & 92 \\
\hline 501 & - & - & - & - & - & - & - & - & - & - & 3 & 167 & 3 & 3 & 502 & 1 & 166 & 169 & 250 & 81 & 3 & 167 & 3 & 3 & 502 & 1 & 166 & 169 & 332 & 250 & - \\
\hline 502 & - & - & - & - & - & - & - & - & - & - & 2 & 251 & 2 & 2 & 503 & 1 & 250 & 251 & 250 & - & 2 & 251 & 2 & 2 & 503 & 1 & 250 & 251 & 500 & 250 & - \\
\hline 504 & 63 & 8 & 7 & 63 & 60 & 3 & 21 & 106 & 251 & 145 & 14 & 36 & 5 & 15 & 182 & 1 & 35 & 75 & 251 & 176 & 14 & 36 & 5 & 15 & 182 & 1 & 35 & 75 & 75 & 251 & 176 \\
\hline 505 & - & - & - & - & - & - & - & - & - & - & 5 & 101 & 5 & 15 & 506 & 1 & 100 & 140 & 252 & 112 & 5 & 101 & 5 & 15 & 506 & 1 & 100 & 140 & 200 & 252 & 52 \\
\hline 506 & - & - & - & - & - & - & - & - & - & - & 2 & 253 & 2 & 2 & 507 & 1 & 252 & 253 & 252 & - & 2 & 253 & 2 & 2 & 507 & 1 & 252 & 253 & 504 & 252 & - \\
\hline
\end{tabular}




\begin{tabular}{|c|c|c|c|c|c|c|c|c|c|c|c|c|c|c|c|c|c|c|c|c|c|c|c|c|c|c|c|c|c|c|c|}
\hline \multirow[b]{2}{*}{$N$} & \multicolumn{10}{|c|}{ EG1 } & \multicolumn{10}{|c|}{ EG2 } & \multicolumn{11}{|c|}{ EG3 } \\
\hline & $n$ & $l$ & $m$ & $g$ & $I$ & $t$ & $F$ & $T$ & $\rho$ & $D 1$ & $n$ & $l$ & $m$ & $g$ & $I$ & $t$ & $F$ & $T$ & $\rho$ & $D 2$ & $n$ & $l$ & $m$ & $g$ & $I$ & $t$ & $F$ & $T$ & $T_{M}$ & $\rho$ & D3 \\
\hline 507 & - & - & - & - & - & - & - & - & - & - & 3 & 169 & 3 & 3 & 508 & 1 & 168 & 171 & 253 & 82 & 3 & 169 & 3 & 3 & 508 & 1 & 168 & 171 & 336 & 253 & - \\
\hline 508 & 508 & 1 & 10 & 511 & 13 & 28 & 23 & 105 & 253 & 148 & 127 & 4 & 8 & 127 & 37 & 13 & 47 & 80 & 253 & 173 & 127 & 4 & 8 & 127 & 37 & 11 & 40 & 81 & 81 & 253 & 172 \\
\hline 510 & 510 & 1 & 10 & 511 & 14 & 28 & 23 & 105 & 254 & 149 & 15 & 34 & 5 & 15 & 172 & 1 & 33 & 73 & 254 & 181 & 15 & 34 & 5 & 15 & 172 & 1 & 33 & 73 & 73 & 254 & 181 \\
\hline 511 & 511 & 1 & 10 & 511 & 17 & 28 & 23 & 105 & 255 & 150 & 511 & 1 & 10 & 511 & 17 & 52 & 46 & 80 & 255 & 175 & 511 & 1 & 10 & 511 & 17 & 46 & 41 & 81 & 82 & 255 & 173 \\
\hline 512 & - & - & - & - & - & - & - & - & - & - & 8 & 64 & 5 & 15 & 320 & 1 & 63 & 103 & 255 & 152 & 8 & 64 & 5 & 15 & 320 & 1 & 63 & 103 & 126 & 255 & 129 \\
\hline 513 & - & - & - & - & - & - & - & - & - & - & 3 & 171 & 3 & 3 & 514 & 1 & 170 & 173 & 256 & 83 & 9 & 57 & 7 & 63 & 400 & 2 & 112 & 249 & 249 & 256 & 7 \\
\hline 514 & - & - & - & - & - & - & - & - & - & - & 2 & 257 & 2 & 2 & 515 & 1 & 256 & 257 & 256 & - & 2 & 257 & 2 & 2 & 515 & 1 & 256 & 257 & 512 & 256 & - \\
\hline 515 & - & - & - & - & - & - & - & - & - & - & 5 & 103 & 5 & 15 & 516 & 1 & 102 & 142 & 257 & 115 & 5 & 103 & 5 & 15 & 516 & 1 & 102 & 142 & 204 & 257 & 53 \\
\hline 516 & - & - & - & - & - & - & - & - & - & - & 12 & 43 & 5 & 15 & 215 & 1 & 42 & 82 & 257 & 175 & 12 & 43 & 5 & 15 & 215 & 1 & 42 & 82 & 84 & 257 & 173 \\
\hline 517 & - & - & - & - & - & - & - & - & - & - & - & - & - & - & - & - & - & - & - & - & - & - & - & - & - & - & - & - & - & - & - \\
\hline 518 & - & - & - & - & - & - & - & - & - & - & 14 & 37 & 5 & 15 & 187 & 1 & 36 & 76 & 258 & 182 & 14 & 37 & 5 & 15 & 187 & 1 & 36 & 76 & 76 & 258 & 182 \\
\hline 519 & - & - & - & - & - & - & - & - & - & - & 3 & 173 & 3 & 3 & 520 & 1 & 172 & 175 & 259 & 84 & 3 & 173 & 3 & 3 & 520 & 1 & 172 & 175 & 344 & 259 & - \\
\hline 520 & 40 & 13 & 9 & 255 & 117 & 1 & 12 & 1688 & 259 & - & 8 & 65 & 5 & 15 & 325 & 1 & 64 & 104 & 259 & 155 & 8 & 65 & 5 & 15 & 325 & 1 & 64 & 104 & 128 & 259 & 131 \\
\hline 524 & - & - & - & - & - & - & - & - & - & - & 4 & 131 & 3 & 4 & 393 & 1 & 130 & 135 & 261 & 126 & 4 & 131 & 3 & 4 & 393 & 1 & 130 & 135 & 260 & 261 & 1 \\
\hline 525 & 105 & 5 & 8 & 127 & 43 & 5 & 22 & 139 & 262 & 123 & 15 & 35 & 5 & 15 & 177 & 1 & 34 & 74 & 262 & 188 & 15 & 35 & 5 & 15 & 177 & 1 & 34 & 74 & 74 & 262 & 188 \\
\hline 526 & - & - & - & - & - & - & - & - & - & - & 2 & 263 & 2 & 2 & 527 & 1 & 262 & 263 & 262 & - & 2 & 263 & 2 & 2 & 527 & 1 & 262 & 263 & 524 & 262 & - \\
\hline 527 & 31 & 17 & 6 & 31 & 106 & 1 & 16 & 129 & 263 & 134 & 31 & 17 & 6 & 31 & 106 & 3 & 48 & 79 & 263 & 184 & 31 & 17 & 6 & 31 & 106 & 2 & 32 & 83 & 83 & 263 & 180 \\
\hline 528 & 24 & 22 & 7 & 63 & 154 & 1 & 21 & 311 & 263 & - & 12 & 44 & 5 & 15 & 220 & 1 & 43 & 83 & 263 & 180 & 12 & 44 & 5 & 15 & 220 & 1 & 43 & 83 & 86 & 263 & 177 \\
\hline 529 & - & - & - & - & - & - & - & - & - & - & - & - & - & - & - & - & - & - & - & - & - & - & - & - & - & - & - & - & - & - & - \\
\hline 530 & - & - & - & - & - & - & - & - & - & - & 5 & 106 & 5 & 15 & 531 & 1 & 105 & 145 & 264 & 119 & 10 & 53 & 6 & 31 & 319 & 1 & 52 & 165 & 165 & 264 & 99 \\
\hline 531 & - & - & - & - & - & - & - & - & - & - & 3 & 177 & 3 & 3 & 532 & 1 & 176 & 179 & 265 & 86 & 9 & 59 & 7 & 63 & 414 & 2 & 116 & 253 & 253 & 265 & 12 \\
\hline 532 & 28 & 19 & 6 & 31 & 115 & 1 & 18 & 131 & 265 & 134 & 14 & 38 & 5 & 15 & 192 & 1 & 37 & 77 & 265 & 188 & 14 & 38 & 5 & 15 & 192 & 1 & 37 & 77 & 77 & 265 & 188 \\
\hline 533 & - & - & - & - & - & - & - & - & - & - & - & - & - & - & - & - & - & - & - & - & - & - & - & - & - & - & - & - & - & - & - \\
\hline 534 & - & - & - & - & - & - & - & - & - & - & 6 & 89 & 4 & 7 & 357 & 1 & 88 & 100 & 266 & 166 & 6 & 89 & 4 & 7 & 357 & 1 & 88 & 100 & 176 & 266 & 90 \\
\hline 535 & - & - & - & - & - & - & - & - & - & - & 5 & 107 & 5 & 15 & 536 & 1 & 106 & 146 & 267 & 121 & 5 & 107 & 5 & 15 & 536 & 1 & 106 & 146 & 212 & 267 & 55 \\
\hline 536 & - & - & - & - & - & - & - & - & - & - & 8 & 67 & 5 & 15 & 335 & 1 & 66 & 106 & 267 & 161 & 8 & 67 & 5 & 15 & 335 & 1 & 66 & 106 & 132 & 267 & 135 \\
\hline 537 & - & - & - & - & - & - & - & - & - & - & 3 & 179 & 3 & 3 & 538 & 1 & 178 & 181 & 268 & 87 & 3 & 179 & 3 & 3 & 538 & 1 & 178 & 181 & 356 & 268 & - \\
\hline
\end{tabular}




\begin{tabular}{|c|c|c|c|c|c|c|c|c|c|c|c|c|c|c|c|c|c|c|c|c|c|c|c|c|c|c|c|c|c|c|c|}
\hline \multirow[b]{2}{*}{$N$} & \multicolumn{10}{|c|}{ EG1 } & \multicolumn{10}{|c|}{ EG2 } & \multicolumn{11}{|c|}{ EG3 } \\
\hline & $n$ & $l$ & $m$ & $g$ & $I$ & $t$ & $F$ & $T$ & $\rho$ & $D 1$ & $n$ & $l$ & $m$ & $g$ & $I$ & $t$ & $F$ & $T$ & $\rho$ & $D 2$ & $n$ & $l$ & $m$ & $g$ & I & $t$ & $F$ & $T$ & $T_{M}$ & $\rho$ & D3 \\
\hline 538 & - & - & - & - & - & - & - & - & - & - & 2 & 269 & 2 & 2 & 539 & 1 & 268 & 269 & 268 & - & 2 & 269 & 2 & 2 & 539 & 1 & 268 & 269 & 536 & 268 & - \\
\hline 539 & - & - & - & - & - & - & - & - & - & - & 7 & 77 & 4 & 7 & 310 & 1 & 76 & 88 & 269 & 181 & 7 & 77 & 4 & 7 & 310 & 1 & 76 & 88 & 152 & 269 & 117 \\
\hline 540 & 30 & 18 & 6 & 31 & 110 & 1 & 17 & 130 & 269 & 139 & 15 & 36 & 5 & 15 & 182 & 1 & 35 & 75 & 269 & 194 & 15 & 36 & 5 & 15 & 182 & 1 & 35 & 75 & 75 & 269 & 194 \\
\hline 542 & - & - & - & - & - & - & - & - & - & - & 2 & 271 & 2 & 2 & 543 & 1 & 270 & 271 & 270 & - & 2 & 271 & 2 & 2 & 543 & 1 & 270 & 271 & 540 & 270 & - \\
\hline 543 & - & - & - & - & - & - & - & - & - & - & 3 & 181 & 3 & 3 & 544 & 1 & 180 & 183 & 271 & 88 & 3 & 181 & 3 & 3 & 544 & 1 & 180 & 183 & 360 & 271 & - \\
\hline 544 & - & - & - & - & - & - & - & - & - & - & 8 & 68 & 5 & 15 & 340 & 1 & 67 & 107 & 271 & 164 & 8 & 68 & 5 & 15 & 340 & 1 & 67 & 107 & 134 & 271 & 137 \\
\hline 545 & - & - & - & - & - & - & - & - & - & - & 5 & 109 & 5 & 15 & 546 & 1 & 108 & 148 & 272 & 124 & 5 & 109 & 5 & 15 & 546 & 1 & 108 & 148 & 216 & 272 & 56 \\
\hline 546 & 42 & 13 & 7 & 63 & 93 & 1 & 12 & 302 & 272 & - & 14 & 39 & 5 & 15 & 197 & 1 & 38 & 78 & 272 & 194 & 14 & 39 & 5 & 15 & 197 & 1 & 38 & 78 & 78 & 272 & 194 \\
\hline 548 & - & - & - & - & - & - & - & - & - & - & 4 & 137 & 3 & 4 & 411 & 1 & 136 & 141 & 273 & 132 & 4 & 137 & 3 & 4 & 411 & 1 & 136 & 141 & 272 & 273 & 1 \\
\hline 549 & - & - & - & - & - & - & - & - & - & - & 3 & 183 & 3 & 3 & 550 & 1 & 182 & 185 & 274 & 89 & 9 & 61 & 7 & 63 & 428 & 2 & 120 & 257 & 257 & 274 & 17 \\
\hline 550 & - & - & - & - & - & - & - & - & - & - & 5 & 110 & 5 & 15 & 551 & 1 & 109 & 149 & 274 & 125 & 10 & 55 & 6 & 31 & 331 & 1 & 54 & 167 & 167 & 274 & 107 \\
\hline 551 & - & - & - & - & - & - & - & - & - & - & - & - & - & - & - & - & - & - & - & - & - & - & - & - & - & - & - & - & - & - & - \\
\hline 552 & 24 & 23 & 7 & 63 & 161 & 1 & 22 & 312 & 275 & - & 12 & 46 & 5 & 15 & 230 & 1 & 45 & 85 & 275 & 190 & 12 & 46 & 5 & 15 & 230 & 1 & 45 & 85 & 90 & 275 & 185 \\
\hline 553 & - & - & - & - & - & - & - & - & - & - & 7 & 79 & 4 & 7 & 318 & 1 & 78 & 90 & 276 & 186 & 7 & 79 & 4 & 7 & 318 & 1 & 78 & 90 & 156 & 276 & 120 \\
\hline 554 & - & - & - & - & - & - & - & - & - & - & 2 & 277 & 2 & 2 & 555 & 1 & 276 & 277 & 276 & - & 2 & 277 & 2 & 2 & 555 & 1 & 276 & 277 & 552 & 276 & - \\
\hline 555 & - & - & - & - & - & - & - & - & - & - & 15 & 37 & 5 & 15 & 187 & 1 & 36 & 76 & 277 & 201 & 15 & 37 & 5 & 15 & 187 & 1 & 36 & 76 & 76 & 277 & 201 \\
\hline 556 & - & - & - & - & - & - & - & - & - & - & 4 & 139 & 3 & 4 & 417 & 1 & 138 & 143 & 277 & 134 & 4 & 139 & 3 & 4 & 417 & 1 & 138 & 143 & 276 & 277 & 1 \\
\hline 558 & 31 & 18 & 6 & 31 & 112 & 1 & 17 & 130 & 278 & 148 & 31 & 18 & 6 & 31 & 112 & 3 & 51 & 82 & 278 & 196 & 31 & 18 & 6 & 31 & 112 & 2 & 34 & 85 & 85 & 278 & 193 \\
\hline 559 & - & - & - & - & - & - & - & - & - & - & - & - & - & - & - & - & - & - & - & - & - & - & - & - & - & - & - & - & - & - & - \\
\hline 560 & 28 & 20 & 6 & 31 & 121 & 1 & 19 & 132 & 279 & 147 & 14 & 40 & 5 & 15 & 202 & 1 & 39 & 79 & 279 & 200 & 14 & 40 & 5 & 15 & 202 & 1 & 39 & 79 & 79 & 279 & 200 \\
\hline 561 & 51 & 11 & 9 & 255 & 102 & 2 & 20 & 839 & 280 & - & 3 & 187 & 3 & 3 & 562 & 1 & 186 & 189 & 280 & 91 & 51 & 11 & 9 & 255 & 102 & 10 & 106 & 230 & 230 & 280 & 50 \\
\hline 562 & - & - & - & - & - & - & - & - & - & - & 2 & 281 & 2 & 2 & 563 & 1 & 280 & 281 & 280 & - & 2 & 281 & 2 & 2 & 563 & 1 & 280 & 281 & 560 & 280 & - \\
\hline 564 & - & - & - & - & - & - & - & - & - & - & 12 & 47 & 5 & 15 & 235 & 1 & 46 & 86 & 281 & 195 & 12 & 47 & 5 & 15 & 235 & 1 & 46 & 86 & 92 & 281 & 189 \\
\hline 565 & - & - & - & - & - & - & - & - & - & - & 5 & 113 & 5 & 15 & 566 & 1 & 112 & 152 & 282 & 130 & 5 & 113 & 5 & 15 & 566 & 1 & 112 & 152 & 224 & 282 & 58 \\
\hline 566 & - & - & - & - & - & - & - & - & - & - & 2 & 283 & 2 & 2 & 567 & 1 & 282 & 283 & 282 & - & 2 & 283 & 2 & 2 & 567 & 1 & 282 & 283 & 564 & 282 & - \\
\hline 567 & 63 & 9 & 7 & 63 & 67 & 2 & 16 & 153 & 283 & 130 & 63 & 9 & 7 & 63 & 67 & 6 & 50 & 84 & 283 & 199 & 63 & 9 & 7 & 63 & 67 & 5 & 42 & 85 & 85 & 283 & 198 \\
\hline 568 & - & - & - & - & - & - & - & - & - & - & 8 & 71 & 5 & 15 & 355 & 1 & 70 & 110 & 283 & 173 & 8 & 71 & 5 & 15 & 355 & 1 & 70 & 110 & 140 & 283 & 143 \\
\hline 570 & 30 & 19 & 6 & 31 & 116 & 1 & 18 & 131 & 284 & 153 & 15 & 38 & 5 & 15 & 192 & 1 & 37 & 77 & 284 & 207 & 15 & 38 & 5 & 15 & 192 & 1 & 37 & 77 & 77 & 284 & 207 \\
\hline
\end{tabular}




\begin{tabular}{|c|c|c|c|c|c|c|c|c|c|c|c|c|c|c|c|c|c|c|c|c|c|c|c|c|c|c|c|c|c|c|c|}
\hline \multirow[b]{2}{*}{$N$} & \multicolumn{10}{|c|}{ EG1 } & \multicolumn{10}{|c|}{ EG2 } & \multicolumn{11}{|c|}{ EG3 } \\
\hline & $n$ & $l$ & $m$ & $g$ & $I$ & $t$ & $F$ & $T$ & $\rho$ & $D 1$ & $n$ & $l$ & $m$ & $g$ & $I$ & $t$ & $F$ & $T$ & $\rho$ & D2 & $n$ & $l$ & $m$ & $g$ & $I$ & $t$ & $F$ & $T$ & $T_{M}$ & $\rho$ & D3 \\
\hline 572 & - & - & - & - & - & - & - & - & - & - & 4 & 143 & 3 & 4 & 429 & 1 & 142 & 147 & 285 & 138 & 4 & 143 & 3 & 4 & 429 & 1 & 142 & 147 & 284 & 285 & 1 \\
\hline 573 & - & - & - & - & - & - & - & - & - & - & 3 & 191 & 3 & 3 & 574 & 1 & 190 & 193 & 286 & 93 & 3 & 191 & 3 & 3 & 574 & 1 & 190 & 193 & 380 & 286 & - \\
\hline 574 & - & - & - & - & - & - & - & - & - & - & 14 & 41 & 5 & 15 & 207 & 1 & 40 & 80 & 286 & 206 & 14 & 41 & 5 & 15 & 207 & 1 & 40 & 80 & 80 & 286 & 206 \\
\hline 575 & - & - & - & - & - & - & - & - & - & - & 5 & 115 & 5 & 15 & 576 & 1 & 114 & 154 & 287 & 133 & 5 & 115 & 5 & 15 & 576 & 1 & 114 & 154 & 228 & 287 & 59 \\
\hline 576 & 24 & 24 & 7 & 63 & 168 & 1 & 23 & 313 & 287 & - & 12 & 48 & 5 & 15 & 240 & 1 & 47 & 87 & 287 & 200 & 12 & 48 & 5 & 15 & 240 & 1 & 47 & 87 & 94 & 287 & 193 \\
\hline 578 & - & - & - & - & - & - & - & - & - & - & 2 & 289 & 2 & 2 & 579 & 1 & 288 & 289 & 288 & - & 2 & 289 & 2 & 2 & 579 & 1 & 288 & 289 & 576 & 288 & - \\
\hline 579 & - & - & - & - & - & - & - & - & - & - & 3 & 193 & 3 & 3 & 580 & 1 & 192 & 195 & 289 & 94 & 3 & 193 & 3 & 3 & 580 & 1 & 192 & 195 & 384 & 289 & - \\
\hline 580 & - & - & - & - & - & - & - & - & - & - & 4 & 145 & 3 & 4 & 435 & 1 & 144 & 149 & 289 & 140 & 20 & 29 & 7 & 63 & 203 & 3 & 84 & 169 & 169 & 289 & 120 \\
\hline 581 & - & - & - & - & - & - & - & - & - & - & 7 & 83 & 4 & 7 & 334 & 1 & 82 & 94 & 290 & 196 & 7 & 83 & 4 & 7 & 334 & 1 & 82 & 94 & 164 & 290 & 126 \\
\hline 582 & - & - & - & - & - & - & - & - & - & - & 6 & 97 & 4 & 7 & 389 & 1 & 96 & 108 & 290 & 182 & 6 & 97 & 4 & 7 & 389 & 1 & 96 & 108 & 192 & 290 & 98 \\
\hline 583 & - & - & - & - & - & - & - & - & - & - & - & - & - & - & - & - & - & - & - & - & - & - & - & - & - & - & - & - & - & - & - \\
\hline 584 & 73 & 8 & 10 & 511 & 84 & 3 & 21 & 1260 & 291 & - & 8 & 73 & 5 & 15 & 365 & 1 & 72 & 112 & 291 & 179 & 8 & 73 & 5 & 15 & 365 & 1 & 72 & 112 & 144 & 291 & 147 \\
\hline 585 & 585 & 1 & 13 & 4095 & 19 & 28 & 23 & 1208 & 292 & - & 15 & 39 & 5 & 15 & 197 & 1 & 38 & 78 & 292 & 214 & 15 & 39 & 5 & 15 & 197 & 1 & 38 & 78 & 78 & 292 & 214 \\
\hline 588 & 28 & 21 & 6 & 31 & 127 & 1 & 20 & 133 & 293 & 160 & 14 & 42 & 5 & 15 & 212 & 1 & 41 & 81 & 293 & 212 & 14 & 42 & 5 & 15 & 212 & 1 & 41 & 81 & 82 & 293 & 211 \\
\hline 589 & 31 & 19 & 6 & 31 & 118 & 1 & 18 & 131 & 294 & 163 & 31 & 19 & 6 & 31 & 118 & 3 & 54 & 85 & 294 & 209 & 31 & 19 & 6 & 31 & 118 & 2 & 36 & 87 & 87 & 294 & 207 \\
\hline 590 & - & - & - & - & - & - & - & - & - & - & 5 & 118 & 5 & 15 & 591 & 1 & 117 & 157 & 294 & 137 & 10 & 59 & 6 & 31 & 355 & 1 & 58 & 171 & 171 & 294 & 123 \\
\hline 591 & - & - & - & - & - & - & - & - & - & - & 3 & 197 & 3 & 3 & 592 & 1 & 196 & 199 & 295 & 96 & 3 & 197 & 3 & 3 & 592 & 1 & 196 & 199 & 392 & 295 & - \\
\hline 592 & - & - & - & - & - & - & - & - & - & - & 8 & 74 & 5 & 15 & 370 & 1 & 73 & 113 & 295 & 182 & 8 & 74 & 5 & 15 & 370 & 1 & 73 & 113 & 146 & 295 & 149 \\
\hline 594 & - & - & - & - & - & - & - & - & - & - & 6 & 99 & 4 & 7 & 397 & 1 & 98 & 110 & 296 & 186 & 6 & 99 & 4 & 7 & 397 & 1 & 98 & 110 & 196 & 296 & 100 \\
\hline 595 & 595 & 1 & 12 & 2047 & 17 & 28 & 23 & 529 & 297 & - & 7 & 85 & 4 & 7 & 342 & 1 & 84 & 96 & 297 & 201 & 7 & 85 & 4 & 7 & 342 & 1 & 84 & 96 & 168 & 297 & 129 \\
\hline 596 & - & - & - & - & - & - & - & - & - & - & 4 & 149 & 3 & 4 & 447 & 1 & 148 & 153 & 297 & 144 & 4 & 149 & 3 & 4 & 447 & 1 & 148 & 153 & 296 & 297 & 1 \\
\hline 597 & - & - & - & - & - & - & - & - & - & - & 3 & 199 & 3 & 3 & 598 & 1 & 198 & 201 & 298 & 97 & 3 & 199 & 3 & 3 & 598 & 1 & 198 & 201 & 396 & 298 & - \\
\hline 598 & - & - & - & - & - & - & - & - & - & - & 2 & 299 & 2 & 2 & 599 & 1 & 298 & 299 & 298 & - & 2 & 299 & 2 & 2 & 599 & 1 & 298 & 299 & 596 & 298 & - \\
\hline 600 & 30 & 20 & 6 & 31 & 122 & 1 & 19 & 132 & 299 & 167 & 15 & 40 & 5 & 15 & 202 & 1 & 39 & 79 & 299 & 220 & 15 & 40 & 5 & 15 & 202 & 1 & 39 & 79 & 79 & 299 & 220 \\
\hline
\end{tabular}




\section{Appendix B. Elliptic and hyperelliptic curve selection}

We describe how the elliptic curve E186 was selected, and how a random instance of the ECDLP in E186 was generated and reduced to an instance of the HCDLP in C186. The elliptic curves E161, E180, E217, E248, E300 and the corresponding hyperelliptic curves listed in Appendix $\mathrm{C}$ were generated in an analogous manner. Note that the hyperelliptic curves produced by the GHS reduction are not unique - we merely list the hyperelliptic curves generated by an invocation of Hess's KASH program [18]. Including the hyperelliptic curves and divisors will assist those who wish to implement the index-calculus methods for the HCDLP without first having to perform the complicated GHS reduction. The elliptic curve E161-2 was generated verifiably at random by selecting the curve $E_{b}: y^{2}+x y=$ $x^{3}+x^{2}+b$, where $b$ is the element in $\mathbb{F}_{2^{161}}$ identified (see below) with the smallest integer greater than or equal to $2^{160}$ for which $\# E_{b}\left(\mathbb{F}_{2^{161}}\right)$ is twice a prime. Finally, the elliptic curves E176 and E272 are taken from ANSI X9.62 [1].

\section{Appendix B.1. Elliptic curve generation}

Let $n=31$, and let $q=2^{6}$. Let $a$ be an arbitrary element of trace 1 in $\mathbb{F}_{2186}$. The order of 2 modulo $n$ is $t=5$. Let $s=6$, and let $f_{i}, 0 \leqslant i \leqslant s$, be the monic irreducible factors of $x^{31}-1$ over $\mathbb{F}_{2}$ with $f_{0}(x)=x-1$ (see Theorem 11). Let $\sigma: \mathbb{F}_{q^{n}} \rightarrow \mathbb{F}_{q^{n}}$ be the Frobenius map defined by $x \mapsto x^{q}$. Define

$$
B=\left\{b \in \mathbb{F}_{q^{n}} \backslash \mathbb{F}_{q}:(\sigma+1) f_{i}(\sigma)(b)=0 \text { for some } 1 \leqslant i \leqslant s\right\} .
$$

The elliptic curve E186 was chosen by selecting random elements $b \in B$ until the number

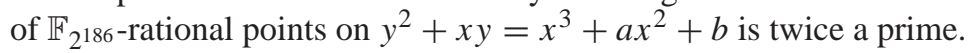

The elements of $\mathbb{F}_{2186}$ are represented as binary polynomials modulo the irreducible polynomial $z^{186}+z^{11}+1$. We identify a 186-bit integer $c=c_{185} 2^{185}+c_{184} 2^{184}+\cdots+c_{0}$ with the element $c_{185} z^{185}+c_{184} z^{184}+\cdots+c_{0}$ of $\mathbb{F}_{2}{ }^{186}$. The defining equation for the elliptic curve E186 is $y^{2}+x y=x^{3}+a x^{2}+b$, where

$$
\begin{aligned}
& a=3 \mathrm{D} 7 \mathrm{D} 03 \mathrm{~F} 4 \mathrm{CB} 539 \mathrm{C} 5 \mathrm{~B} 728 \mathrm{D} 256 \mathrm{DAD} 2 \mathrm{E} 5 \mathrm{E} 8 \mathrm{ADDB} 81 \mathrm{~B} 524 \mathrm{~F} 7 \mathrm{D} 68 \mathrm{D}, \text { and } \\
& b=35108742308 \mathrm{~B} 720 \mathrm{FAABCFFB} 70 \mathrm{D} 33 \mathrm{E} 3840 \mathrm{~F} 8 \mathrm{D} 93323635 \mathrm{~F} 7 \mathrm{E}
\end{aligned}
$$

in hexadecimal notation. The number of $\mathbb{F}_{2^{186}}$-rational points on E186 is $2 r$, where

$$
r=200000000000000000000000 \mathrm{E} 5 \mathrm{BED} 0151962 \mathrm{E} 91 \mathrm{~F} 6 \mathrm{CDF} 581
$$

is prime.

\section{Appendix B.2. ECDLP instance generation}

We selected two points $P$ and $Q$ from E186( $\left.\mathbb{F}_{2186}\right)$ verifiably at random as follows. We first defined 160-bit integers $m_{1}$ and $m_{2}$ to be the 160-bit outputs of the SHA-1 cryptographic hash function with inputs the strings ' ' and 'a', respectively. (These two strings are commonly used as inputs to generate test vectors for hash functions; see [25, Table 9.6].) We identify a 160-bit integer $c=c_{159} 2^{159}+c_{158} 2^{158}+\cdots+c_{0}$ with the element $c_{159} z^{159}+c_{158} z^{158}+\cdots+c_{0}$ of $\mathbb{F}_{2}{ }^{186}$. Then, for each $i \in\{1,2\}$, we define $n_{i}$ to be the smallest integer greaater than or equal to $m_{i}$ for which the field element corresponding to $n_{i}$ is the $x$-coordinate of some point of order not equal to 2 in E186 $\left(\mathbb{F}_{2186}\right)$; for such an $n_{i}$ we arbitrarily select one of the two possible $y$-coordinates to obtain two points $P^{\prime}$ and $Q^{\prime}$, and then we set $P=2 P^{\prime}$ and $Q=2 Q^{\prime}$. In this way, we derive the following two points of 
order $r$ :

$$
\begin{aligned}
& P=(6 \mathrm{FE} 4 \mathrm{D} 23 \mathrm{FBAFBAF} 66317050 \mathrm{~A} 0 \mathrm{D} 102 \mathrm{E} 23075572174 \mathrm{ADC} 304 \text {, } \\
& \text { 24E2CB9E1DAF2 61EA2 5FD } 0413 \text { F 85CF } 067 \text { DB5FE50F } 484 \text { 9B2); } \\
& Q=(\text { EFD00F993676085F97D9BB9117E00A34F6185104629F42, } \\
& \text { 1EBBB1F 4 36A53B00B4C74A93CF6E613F3C60D566BDB9653). }
\end{aligned}
$$

The ECDLP challenge is to find the integer $\lambda \in[0, r-1]$ such that $Q=\lambda P$. Note that since $P$ and $Q$ were (pseudo)randomly generated, the discrete logarithm $\lambda$ is not known by us a priori.

\section{Appendix B.3. HCDLP instance generation}

Hess's KASH program [18] for the Weil restriction represents elliptic curve points as zero divisors. For technical reasons, it excludes the point at infinity from occurring in the support of the divisors. Thus, instead of representing an elliptic curve point $P$ by a zero divisor $(P)-(\infty)$, we represent $P$ by the equivalent zero divisor $(P+R)-(R)$, where $R$ is an arbitrary point on the curve. We arbitrarily selected the following point of order $r$ :

$$
R=(3 \mathrm{~A} 9 \mathrm{EE} 0 \text { 9AEC0996B 46F3680D80835FF3081D795A93AB58FF, }
$$
FC867E29309F63717894B647A611E743919B511E204862).

Let $P_{1}=P+R, P_{2}=Q+R$ and $P_{3}=R$. Hess's KASH program was used to reduce (E186, $\left.P_{1}, P_{2}, P_{3}\right)$ to $\left(\mathrm{C} 186, D_{1}, D_{2}, D_{3}\right)$, where $\mathrm{C} 186$ is a genus-31 hyperelliptic curve over $\mathbb{F}_{2^{6}}$ and $D_{1}, D_{2}, D_{3}$ are divisors in $J_{\mathrm{C} 186}\left(\mathbb{F}_{2^{6}}\right)$. The elements of $\mathbb{F}_{2^{6}}$ are represented as binary polynomials modulo the irreducible polynomial $w^{6}+w^{4}+w^{3}+w+1$. The Weierstrass equation for the hyperelliptic curve C186 is $v^{2}+h(u) v=f(u)$, where

$$
\begin{aligned}
& f(u)=w^{30} u^{63}+w^{10} u^{62}+w^{40} u^{60}+w^{54} u^{56}+w^{23} u^{48}+w^{26} ; \\
& h(u)=w^{15} u^{31}+w u^{30}+w^{21} u^{28}+w^{59} u^{24}+w^{41} u^{16}+w^{10} .
\end{aligned}
$$

The divisors $D_{1}, D_{2}$ and $D_{3}$ are:

$$
\begin{aligned}
D_{1}=\operatorname{div}\left(u^{31}\right. & +w^{32} u^{30}+w^{58} u^{29}+w^{57} u^{28}+w^{11} u^{27}+w^{25} u^{26}+w^{39} u^{24}+w^{37} u^{23}+w^{59} u^{22} \\
& +w^{19} u^{21}+w^{3} u^{20}+w^{45} u^{19}+w^{47} u^{18}+w u^{16}+w^{40} u^{15}+w^{6} u^{14}+w^{53} u^{13} \\
& +w^{48} u^{12}+w^{30} u^{11}+w^{33} u^{10}+w^{19} u^{9}+w^{55} u^{8}+w^{28} u^{7}+w^{7} u^{6}+w^{20} u^{5} \\
& +w^{5} u^{4}+w^{38} u^{3}+w^{29} u^{2}+w^{60} u+w^{11}, w^{36} u^{30}+w^{28} u^{29}+w^{27} u^{28}+w^{24} u^{27} \\
& +w^{12} u^{26}+w^{58} u^{25}+w^{62} u^{24}+w^{8} u^{23}+w^{13} u^{22}+w^{41} u^{21}+w^{22} u^{20}+w^{11} u^{19} \\
& +w^{40} u^{18}+w^{26} u^{17}+w^{39} u^{16}+w^{19} u^{15}+w^{39} u^{14}+w^{43} u^{13}+w^{6} u^{8}+w^{53} u^{7} \\
& \left.+w^{42} u^{6}+w^{50} u^{5}+w^{18} u^{4}+w^{2} u^{3}+w^{38} u^{2}+w^{11} u+w\right), \\
D_{2}=\operatorname{div}\left(u^{31}\right. & +w^{9} u^{30}+w^{23} u^{29}+w^{17} u^{28}+w^{23} u^{27}+w^{37} u^{26}+w^{34} u^{25}+w^{25} u^{24} \\
& +w^{46} u^{23}+w^{21} u^{22}+w^{61} u^{21}+w^{42} u^{20}+w^{39} u^{19}+w^{7} u^{18}+w^{43} u^{17}+w^{50} u^{16} \\
& +w^{43} u^{15}+w^{22} u^{14}+w^{24} u^{13}+w^{31} u^{12}+w^{24} u^{11}+w^{5} u^{10}+w^{28} u^{9}+w^{62} u^{8} \\
& +w^{34} u^{7}+u^{6}+w^{45} u^{5}+w^{18} u^{4}+w^{15} u^{3}+w^{54} u^{2}+w^{4} u+1, w^{12} u^{30}+w^{32} u^{29} \\
& +w^{19} u^{28}+w^{62} u^{27}+w^{25} u^{26}+w^{45} u^{25}+w^{50} u^{24}+w^{18} u^{23}+w^{51} u^{22}+w^{21} \\
& +w^{36} u^{20}+w^{5} u^{19}+w^{58} u^{18}+w^{60} u^{17}+w^{22} u^{16}+w^{11} u^{15}+w^{12} u^{14}+w^{25} u^{13} \\
& +w^{47} u^{12}+w^{4} u^{11}+w^{62} u^{9}+w^{60} u^{8}+w^{33} u^{7}+w^{52} u^{6}+w^{21} u^{5}+w^{43} u^{4} \\
& \left.+w^{36} u^{3}+w^{50} u^{2}+w^{5} u+w^{20}\right),
\end{aligned}
$$




$$
\begin{aligned}
D_{3}=\operatorname{div}\left(u^{31}\right. & +w^{54} u^{30}+w^{42} u^{29}+w^{62} u^{28}+w^{38} u^{27}+w^{11} u^{26}+w^{15} u^{25}+w^{2} u^{24} \\
& +w^{62} u^{23}+w^{54} u^{22}+w^{8} u^{21}+w^{53} u^{20}+w^{17} u^{19}+w^{6} u^{18}+u^{17}+w^{51} u^{16} \\
& +w^{22} u^{15}+w^{61} u^{14}+w^{2} u^{13}+w^{61} u^{12}+w^{40} u^{11}+w^{12} u^{10}+w^{14} u^{9}+w^{3} u^{8} \\
& +w^{13} u^{7}+w^{31} u^{6}+w^{60} u^{5}+w^{16} u^{4}+w^{43} u^{3}+w^{3} u^{2}+w^{9} u+w^{7}, w^{25} u^{30} \\
& +w^{24} u^{29}+w^{62} u^{28}+w^{13} u^{27}+w^{17} u^{26}+w^{53} u^{25}+w^{52} u^{24}+w^{43} u^{23}+w^{20} u^{22} \\
& +w^{51} u^{21}+w^{23} u^{20}+w^{59} u^{19}+w^{60} u^{18}+w^{49} u^{17}+w^{20} u^{16}+w^{47} u^{15}+w^{53} u^{14} \\
& +w^{40} u^{13}+w^{49} u^{12}+w^{28} u^{11}+w^{3} u^{10}+w^{6} u^{9}+w^{35} u^{8}+w^{41} u^{7}+w^{6} u^{6} \\
& \left.+w^{46} u^{5}+w^{57} u^{3}+w^{9} u^{2}+w^{21} u+w^{53}\right) .
\end{aligned}
$$

The task is to solve the following discrete logarithm problem in $J_{\mathrm{C} 186}\left(\mathbb{F}_{2^{6}}\right)$ : find the integer $\lambda \in[0, r-1]$ such that $\left(D_{2}-D_{3}\right)=\lambda\left(D_{1}-D_{3}\right)$.

\section{Appendix C. ECDLP challenge parameters}

For an explanation of the notation used in the following tables, see Section 7 and Appendix B.

$$
\begin{aligned}
& \text { E161, } N=161, \mathbb{F}_{2^{161}}=\mathbb{F}_{2}[z] /\left(z^{161}+z^{18}+1\right) \text {, \#E161 }\left(\mathbb{F}_{2^{161}}\right)=2 \cdot r, a=1 \\
& b=1102 \text { A36EE3EEE95C1DDA26A51A954391733728D22 } \\
& r=\text { FFFFFFFFFFFFFFFFFED3F975D827A7D20F8 } 9 \\
& P=\text { (1CBF654BEEF0AE9F525F8E9F5FA1DED1D10C7D781, } \\
& \text { 175984F97695A39291B94B6D 9BD89860C9AF5DF80) } \\
& Q=(\mathrm{AE} 24976 \mathrm{AE} 483 \mathrm{ED} 2 \mathrm{E} 33 \mathrm{~A} 77 \mathrm{FD} 48 \mathrm{~F} 78 \mathrm{DAE} 06 \mathrm{ED} 0 \mathrm{~F} 54 \mathrm{E}, \\
& \text { 186EBA8B979ADAA320D47C7763CFF 8EF810A970EB) } \\
& R=(1 \mathrm{E} 7958 \mathrm{EF} 1 \mathrm{FA} 48 \mathrm{~A} 2 \mathrm{~B} 92889 \mathrm{~B} 44 \text { 2DADE6E9A6A7C173, } \\
& \text { 4EE6671B1A5D69A5578EFE30C05704FA69C78345) }
\end{aligned}
$$

$$
\begin{aligned}
& \text { C161, } q=2^{23}, \mathbb{F}_{2^{23}}=\mathbb{F}_{2}[w] /\left(w^{23}+w^{5}+1\right) \\
& f(u)=w^{6691705} u^{15}+w^{4316786} u^{14}+w^{4857716} u^{12}+w^{4289455} u^{8}+w^{7257339} \\
& h(u)=w^{7540156} u^{7}+w^{4708240} u^{6}+w^{2060647} u^{4}+w^{7822973} \\
& D_{1}=\operatorname{div}\left(u^{7}+w^{111674} u^{6}+w^{6262987} u^{5}+w^{5507868} u^{4}+w^{5024071} u^{3}+w^{7360243} u^{2}\right. \\
& +w^{4982988} u+w^{3476956}, w^{7214579} u^{6}+w^{1039748} u^{5}+w^{5362902} u^{4} \\
& \left.+w^{5575575} u^{3}+w^{6046318} u^{2}+w^{783556} u+w^{7954483}\right) \\
& D_{2}=\operatorname{div}\left(u^{7}+w^{2418740} u^{6}+w^{6332447} u^{5}+w^{5288518} u^{4}+w^{6581623} u^{3}+w^{3461659} u^{2}\right. \\
& +w^{663714} u+w^{2094946}, w^{5819570} u^{6}+w^{5789770} u^{5}+w^{3853008} u^{4} \\
& \left.+w^{3628267} u^{3}+w^{4786898} u^{2}+w^{3463517} u+w^{2504145}\right) \\
& D_{3}=\operatorname{div}\left(u^{7}+w^{7595037} u^{6}+w^{6492024} u^{5}+w^{5128797} u^{4}+w^{1479702} u^{3}+w^{3764869} u^{2}\right. \\
& +w^{2973617} u+w^{3579984}, w^{5819570} u^{6}+w^{5789770} u^{5}+w^{3853008} u^{4} \\
& \left.+w^{3628267} u^{3}+w^{4786898} u^{2}+w^{3463517} u+w^{2504145}\right)
\end{aligned}
$$


E180, $N=180, \mathbb{F}_{2^{180}}=\mathbb{F}_{2}[z] /\left(z^{180}+z^{3}+1\right), \# \mathrm{E} 180\left(\mathbb{F}_{2^{180}}\right)=2 \cdot r$

$a=\mathrm{B} 3 \mathrm{C} 8 \mathrm{~B} 5 \mathrm{AF} 89342 \mathrm{D} 73 \mathrm{C} 9 \mathrm{D} 12 \mathrm{~F} 1 \mathrm{~F} 5 \mathrm{ACDD} 36011626 \mathrm{BBC} 675 \mathrm{C} 1$

$b=990 \mathrm{D} 04982994434 \mathrm{CB} 4 \mathrm{C} 14 \mathrm{DB} 21204025865 \mathrm{~B} 40069225 \mathrm{~B} 2$

$r=800000000000000000000023 \mathrm{ABAE} 178 \mathrm{BD} 70 \mathrm{C} 3 \mathrm{E} 01 \mathrm{FDC} 31$

$P=$ (EE4AB1D7C359522ED9CABE52021DAD0EAF613C1ECE8CB, 670775621CD859D56079BB52298C0A509AB4E689593F9)

$Q=(7 F 13258 \mathrm{D} 03372 \mathrm{C} 8 \mathrm{~A} 571 \mathrm{E} 8 \mathrm{C} 199 \mathrm{DD} 9416 \mathrm{~A} 7642 \mathrm{DDA} 05 \mathrm{C} 515$, $1182432 \mathrm{~B} 1 \mathrm{~B} 3 \mathrm{C} 6 \mathrm{C} 1856 \mathrm{D} 47 \mathrm{~B} 139 \mathrm{~B} 28 \mathrm{E} 003 \mathrm{~B} 6 \mathrm{D} 9 \mathrm{~F} 440574 \mathrm{FF})$ $R=(\mathrm{BAD} 2 \mathrm{E} 0 \mathrm{D} 1 \mathrm{D} 655559 \mathrm{AF} 62 \mathrm{E} 346 \mathrm{BE} 2090135 \mathrm{E} 40 \mathrm{AEC} 22 \mathrm{EE} 5 \mathrm{C} 3$, B5B71A32C4498B8CF0DF6BB90911D91CC16F506D508C5)

C180, $q=2^{12}, \mathbb{F}_{2^{12}}=\mathbb{F}_{2}[w] /\left(w^{12}+w^{7}+w^{6}+w^{5}+w^{3}+w+1\right)$ $f(u)=w^{1977} u^{31}+w^{3517} u^{30}+w^{3954} u^{28}+w^{3666} u^{24}+w^{3749} u^{16}+w^{2880}$ $h(u)=w^{3036} u^{15}+w^{4031} u^{14}+w^{1455} u^{12}+w^{2278} u^{8}+w^{2024}$ $D_{1}=\operatorname{div}\left(u^{15}+w^{913} u^{14}+w^{120} u^{13}+w^{1222} u^{12}+w^{717} u^{11}+w^{1158} u^{10}+w^{406} u^{9}\right.$ $+w^{3864} u^{8}+w^{3391} u^{7}+w^{3302} u^{6}+w^{906} u^{5}+w^{2528} u^{4}+w^{3620} u^{3}+w^{1164} u^{2}$ $+w^{119} u+w^{68}, w^{3862} u^{14}+w^{1139} u^{13}+w^{4055} u^{12}+w^{3324} u^{11}+w^{1436} u^{10}$ $+w^{1968} u^{9}+w^{1488} u^{8}+w^{22} u^{7}+w^{3071} u^{6}+w^{1736} u^{5}+w^{394} u^{4}+w^{1892} u^{3}$ $\left.+w^{3461} u^{2}+w^{923} u+w^{2371}\right)$

$D_{2}=\operatorname{div}\left(u^{15}+w^{2828} u^{14}+w^{2507} u^{13}+w^{2845} u^{12}+w^{3821} u^{11}+w^{550} u^{10}+w^{837} u^{9}\right.$ $+w^{3146} u^{8}+w^{1040} u^{7}+w^{1551} u^{6}+w^{2806} u^{5}+w^{2321} u^{4}+w^{251} u^{3}+w^{3983} u^{2}$ $+w^{1482} u+w^{1796}, w^{2370} u^{14}+w^{3993} u^{13}+w^{2270} u^{12}+w^{3787} u^{11}+w^{2128} u^{10}$ $+w^{2948} u^{9}+w^{72} u^{8}+w^{27} u^{7}+w^{1515} u^{6}+w^{1684} u^{5}+w^{385} u^{4}+w^{3635} u^{3}$ $\left.+w^{1076} u^{2}+w^{1654} u+w^{3081}\right)$

$D_{3}=\operatorname{div}\left(u^{15}+w^{2485} u^{14}+w^{1754} u^{13}+w^{1638} u^{12}+w^{2078} u^{11}+w^{4039} u^{10}+w^{2857} u^{9}\right.$ $+w^{2716} u^{8}+w^{230} u^{7}+w^{1139} u^{6}+w^{1330} u^{5}+w^{851} u^{4}+w^{1926} u^{3}+w^{428} u^{2}$ $+w^{2628} u+w^{2729}, w^{2543} u^{14}+w^{998} u^{13}+w^{37} u^{12}+w^{1097} u^{11}+w^{2830} u^{10}$ $+w^{770} u^{9}+w^{2604} u^{8}+w^{3011} u^{7}+w^{2334} u^{6}+w^{863} u^{5}+w^{1952} u^{4}+w^{1777} u^{3}$ $\left.+w^{1122} u^{2}+w^{1754} u+w^{3677}\right)$ 
E186, $N=186, \mathbb{F}_{2^{186}}=\mathbb{F}_{2}[z] /\left(z^{186}+z^{11}+1\right), \# \mathrm{E} 186\left(\mathbb{F}_{2^{186}}\right)=2 \cdot r$

$a=3 \mathrm{D} 7 \mathrm{D} 03 \mathrm{~F} 4 \mathrm{CB} 539 \mathrm{C} 5 \mathrm{~B} 728 \mathrm{D} 256 \mathrm{DAD} 2 \mathrm{E} 5 \mathrm{E} 8 \mathrm{ADDB} 81 \mathrm{~B} 524 \mathrm{~F} 7 \mathrm{D} 68 \mathrm{D}$

$b=35108742308 \mathrm{~B} 720 \mathrm{FAABCFFB} 70 \mathrm{D} 33 \mathrm{E} 3840 \mathrm{~F} 8 \mathrm{D} 93323635 \mathrm{~F} 7 \mathrm{E}$

$r=200000000000000000000000 \mathrm{E} 5 \mathrm{BED} 0151962 \mathrm{E} 91 \mathrm{~F} 6 \mathrm{CDF} 581$

$P=(6 \mathrm{FE} 4 \mathrm{D} 23 \mathrm{FBAFBAF} 66317050 \mathrm{~A} 0 \mathrm{D} 102 \mathrm{E} 23075572174 \mathrm{ADC} 304$, 24E2CB9E1DAF2 61EA2 5FD 0413 F 85CF 067 DB5FE50F 484 9B2)

$Q=($ EFD00F993676085F97D9BB9117E00A34F6185104629F42,

1EBBB1F 436A53B00B4C74A93CF6E613F3C60D566BDB9653)

$R=(3 \mathrm{~A} 9 \mathrm{EE} 09 \mathrm{AEC} 0996 \mathrm{~B} 46 \mathrm{~F} 3680 \mathrm{D} 80835 \mathrm{FF} 3081 \mathrm{D} 795 \mathrm{~A} 93 \mathrm{AB} 58 \mathrm{FF}$,

FC867E29309F63717894B647A611E743919B511E204862)

C186, $q=64, \mathbb{F}_{2^{6}}=\mathbb{F}_{2}[w] /\left(w^{6}+w^{4}+w^{3}+w+1\right)$

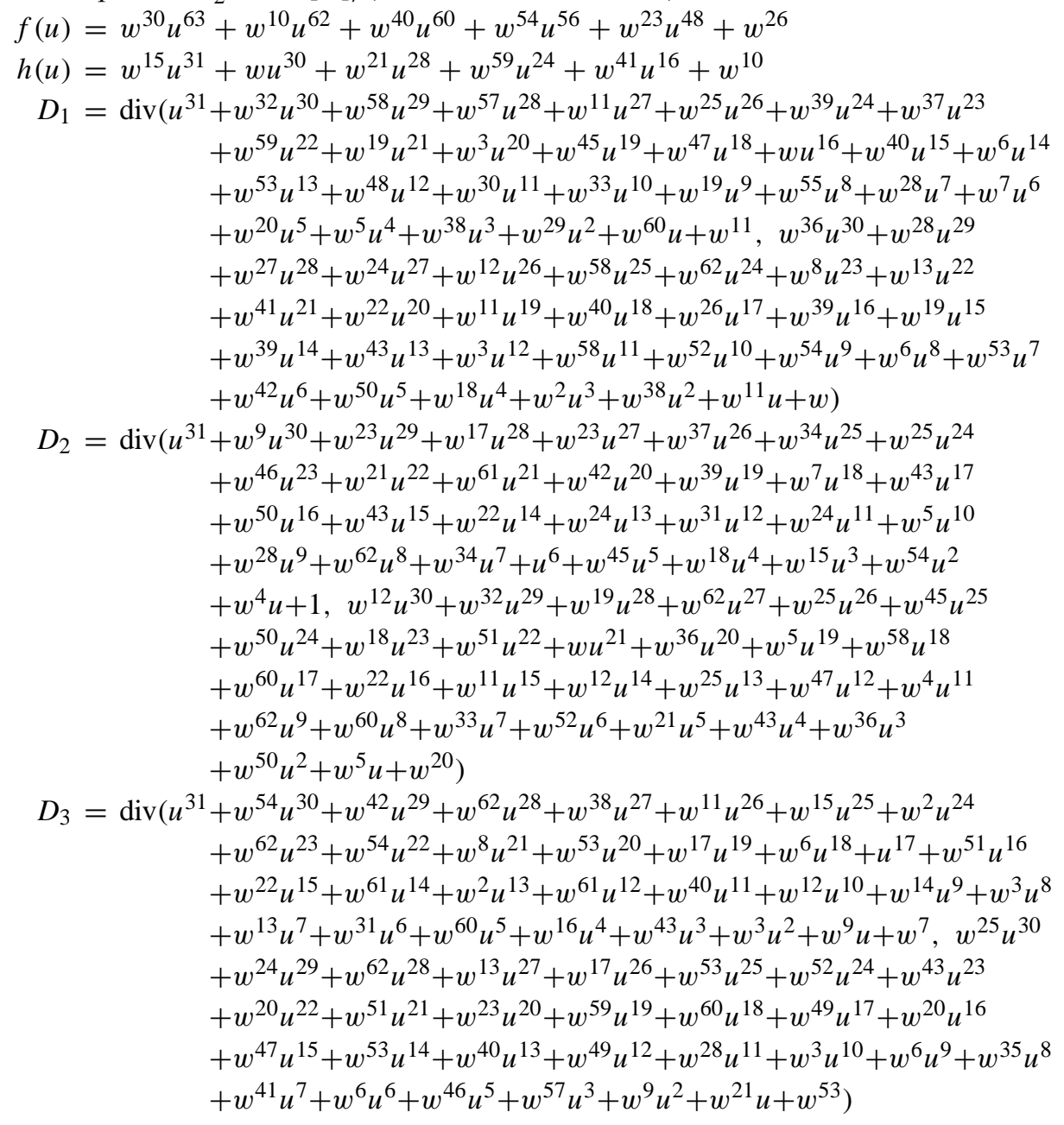


E217, $N=217, \mathbb{F}_{2^{217}}=\mathbb{F}_{2}[z] /\left(z^{217}+z^{45}+1\right), \# \mathrm{E} 217\left(\mathbb{F}_{2^{217}}\right)=2 \cdot r, a=1$

$$
\begin{aligned}
& b=11 \mathrm{E} 8 \mathrm{~F} 97 \mathrm{~F} 344082577 \mathrm{BB} 4 \mathrm{D} 782 \mathrm{D} 3 \mathrm{~F} 433 \mathrm{FBE} 30 \mathrm{D} 8 \mathrm{~F} 3 \mathrm{D} 65684 \mathrm{AE} 499694 \mathrm{BA} \\
& r=10000000000000000000000000000598 \text { FB15077594E7069CED749A1 } \\
& P=\text { (1B3E0A79E37A60852C959F1C776AEA48D328A75F8C683696CE74D55, } \\
& \text { 1CE258C697FC2A36401ADFC3DE84BB7EF5253E142159E79A474EA1) } \\
& Q=(13 \mathrm{AC} 35 \mathrm{CCA} 7052 \mathrm{FE} 1368820 \mathrm{D} 1 \mathrm{CAFC} 23 \mathrm{DB} 5 \mathrm{~F} 004681 \mathrm{~EB} 781 \mathrm{C} 931 \text { 9F 39A1, } \\
& \text { CD31941B339D9E6C6E54759E6C77C9A5FAF0BF5AB928F1EE63668F) } \\
& R=\text { (19524EC31D1843F21AA0D8CF5318D83FA150A17FA519ACAF2E571A5, } \\
& \text { DA7F2D3B1A16E78BF } 070 \text { 4A0 7DD 4FB4 38A0F23DCE8EEC0 4B3A50B2A) }
\end{aligned}
$$

C217, $q=128, \mathbb{F}_{2^{7}}=\mathbb{F}_{2}[w] /\left(w^{7}+w+1\right)$

$$
\begin{aligned}
f(u)=w^{96} u^{63} & +w^{79} u^{62}+w^{24} u^{60}+w^{122} u^{56}+w^{63} u^{48}+w^{60} u^{32}+w^{64} \\
h(u)=w^{48} u^{31} & +w^{74} u^{30}+w^{36} u^{28}+w^{107} u^{24}+w^{11} u^{16}+w^{32} \\
D_{1}=\operatorname{div}\left(u^{31}\right. & +w^{97} u^{30}+w^{66} u^{29}+w^{111} u^{28}+w^{125} u^{27}+w^{58} u^{26}+w^{88} u^{25}+w^{96} u^{24} \\
& +w^{106} u^{23}+w^{68} u^{22}+w u^{21}+w^{93} u^{20}+w^{108} u^{19}+w^{81} u^{18}+w^{107} u^{17} \\
& +w^{78} u^{16}+w^{113} u^{15}+w^{68} u^{14}+w^{115} u^{13}+w^{92} u^{12}+w^{86} u^{11}+w^{67} u^{10} \\
& +w^{73} u^{9}+w^{42} u^{8}+w u^{7}+w^{46} u^{6}+w^{51} u^{5}+w^{116} u^{4}+w^{28} u^{3}+w^{99} u^{2} \\
& +w^{105} u+w^{29}, w^{7} u^{30}+w^{22} u^{29}+w u^{28}+w^{37} u^{27}+w^{114} u^{26}+w^{93} u^{25} \\
& +w^{57} u^{24}+w^{121} u^{23}+w^{117} u^{22}+w^{7} u^{21}+w^{81} u^{20}+w^{98} u^{18}+w^{107} u^{17} \\
& +w^{84} u^{16}+w^{45} u^{15}+w^{86} u^{14}+w^{108} u^{13}+w^{90} u^{12}+w^{50} u^{11}+u^{10} \\
& +w^{111} u^{9}+w^{16} u^{8}+w^{21} u^{7}+w^{44} u^{6}+w^{14} u^{5}+w^{93} u^{4}+w^{36} u^{3} \\
& \left.+w^{118} u^{2}+w^{97} u+w^{3}\right) \\
D_{2}=\operatorname{div}\left(u^{31}\right. & +w^{47} u^{30}+w^{40} u^{29}+w^{39} u^{28}+w^{108} u^{27}+w^{113} u^{26}+w^{99} u^{25}+w^{87} u^{24}
\end{aligned}
$$$$
+w^{84} u^{23}+w^{33} u^{22}+w^{46} u^{21}+w^{34} u^{20}+w^{16} u^{19}+w^{51} u^{18}+w^{39} u^{17}
$$$$
+w^{116} u^{16}+w^{41} u^{15}+w^{50} u^{14}+w^{121} u^{13}+w^{108} u^{12}+w^{82} u^{11}+w^{74} u^{10}
$$$$
+w^{4} u^{9}+w^{118} u^{8}+w^{17} u^{7}+w^{95} u^{6}+w^{13} u^{5}+w^{23} u^{4}+w^{44} u^{3}+w^{29} u^{2}
$$$$
+w^{52} u+w^{7}, w^{105} u^{30}+w^{124} u^{29}+w^{91} u^{28}+w^{92} u^{27}+w^{102} u^{26}+w^{76} u^{25}
$$$$
+w^{2} u^{24}+w^{53} u^{23}+w^{98} u^{22}+w^{24} u^{21}+w^{58} u^{20}+w^{64} u^{19}+w^{76} u^{18}
$$$$
+w^{24} u^{17}+w^{58} u^{16}+w^{5} u^{15}+w^{14} u^{14}+w^{19} u^{13}+w^{33} u^{12}+w^{14} u^{11}
$$$$
+w^{71} u^{10}+w^{61} u^{9}+w^{125} u^{8}+w^{29} u^{7}+w^{73} u^{6}+w^{31} u^{5}+w^{25} u^{4}
$$$$
\left.+w^{115} u^{3}+w^{73} u^{2}+w^{62} u+w^{70}\right)
$$$$
D_{3}=\operatorname{div}\left(u^{31}+w^{111} u^{30}+w^{5} u^{29}+w^{74} u^{28}+w^{44} u^{27}+w^{25} u^{26}+w^{53} u^{25}+w^{15} u^{24}\right.
$$$$
+w^{13} u^{23}+w^{44} u^{22}+w^{23} u^{21}+w^{46} u^{20}+w^{122} u^{19}+w^{52} u^{18}+w^{55} u^{17}
$$$$
+w^{35} u^{16}+w^{81} u^{15}+w^{97} u^{14}+w^{14} u^{13}+w^{89} u^{12}+w^{114} u^{11}+w^{24} u^{10}
$$$$
+w^{33} u^{9}+w^{87} u^{8}+w^{18} u^{7}+w^{83} u^{6}+w^{60} u^{5}+w^{100} u^{4}+w^{125} u^{3}+w^{68} u^{2}
$$$$
+w^{44} u+w^{40}, w^{39} u^{30}+w^{108} u^{29}+w^{51} u^{28}+w^{100} u^{27}+w^{8} u^{26}+w^{43} u^{25}
$$$$
+w^{108} u^{24}+w^{81} u^{23}+w^{12} u^{22}+w^{21} u^{21}+w^{84} u^{20}+w^{15} u^{19}+w^{109} u^{18}
$$$$
+w^{114} u^{17}+w^{25} u^{16}+w^{18} u^{15}+w^{115} u^{14}+w^{122} u^{13}+u^{12}+w^{74} u^{11}
$$$$
+w^{34} u^{10}+w^{60} u^{9}+w^{58} u^{8}+w^{6} u^{7}+w^{80} u^{6}+w^{68} u^{5}+w^{7} u^{4}+w^{6} u^{3}
$$$$
\left.+w^{77} u^{2}+w^{31} u\right)
$$ 
E248, $N=248, \mathbb{F}_{2248}=\mathbb{F}_{2}[z] /\left(z^{248}+z^{15}+z^{14}+z^{10}+1\right), \# \mathrm{E} 248\left(\mathbb{F}_{2248}\right)=2 \cdot r$

$a=\mathrm{C} 65 \mathrm{~B} 1 \mathrm{~B} 2 \mathrm{~B} 48 \mathrm{E} 3 \mathrm{~A} 7 \mathrm{~B} 2 \mathrm{BC} 69 \mathrm{C} 365 \mathrm{~B} 4 \mathrm{CAE} 385 \mathrm{CE} 3 \mathrm{~F} 023 \mathrm{~A} 9742 \mathrm{C} 0 \mathrm{EF} 16$ C40E1D 62ADA2

$b=1649390 \mathrm{E} 2 \mathrm{C} 5 \mathrm{BBA} 8206486 \mathrm{E} 33 \mathrm{D} 3273 \mathrm{AE} 26$ 9EE $568 \mathrm{~F} 91 \mathrm{AC} 46 \mathrm{BC} 86$ A $6 \mathrm{~A} 792 \mathrm{CF} 6 \mathrm{CEA}$

$r=80000000000000000000000000000004 \mathrm{~B} 55 \mathrm{AAB} 05 \mathrm{CC} 2 \mathrm{C} 4 \mathrm{EE} 2 \mathrm{E} 6$ 973D $0 \mathrm{C} 247 \mathrm{E} 01$

$P=$ (4AF353C030AADA55B6FAED2BBF 314EBC28C8B6FB1DFC0 9272 8A28E70F0E73F， 168FADD57DC3046C69A0121310D284A04C 145E0ECB88B91BAD8BE25F58CBE9)

$Q=$ (62231B26EEF12154100D6257F7148AC78C5A61FC129532449 4368A42CA9FD5, 403910538623DE3633D0FD6690E12F 4D 81 D1915D3728C5617CD6A65B92AA41)

$R=$ (D69A12AFCF 86A8CF 9F30051B8C655D050B238A215C19C8512 D41B547C1ADE2, A3FDA75DB34F93EF657FAAB2163AE05E0C C1241175CE1E880841F77A369B8B)

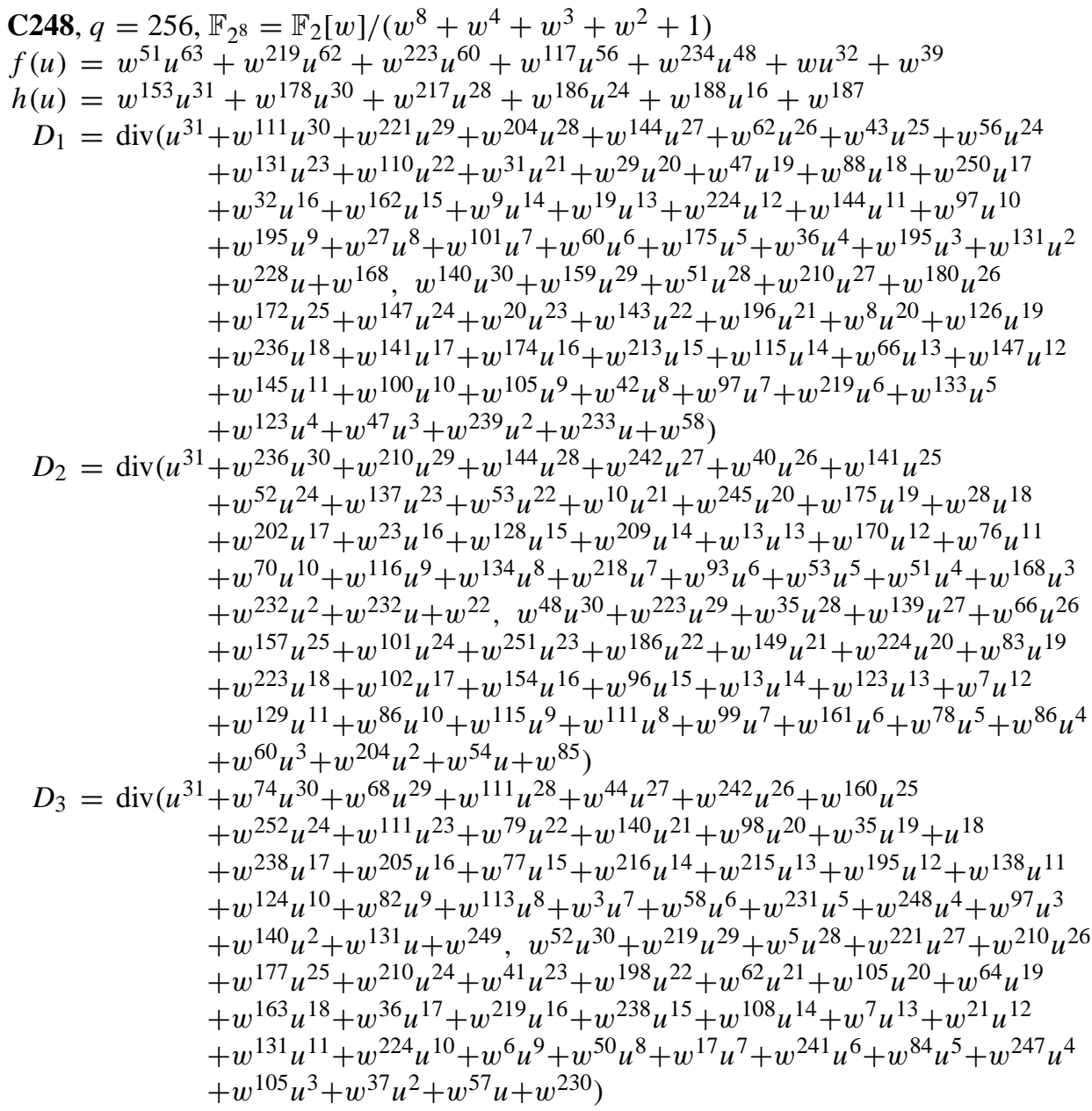


E300, $N=300, \mathbb{F}_{2^{300}}=\mathbb{F}_{2}[z] /\left(z^{300}+z^{5}+1\right), \# \mathrm{E} 300\left(\mathbb{F}_{2^{300}}\right)=2 \cdot r$

$a=8 \mathrm{E} 8 \mathrm{EEC} 356 \mathrm{CB} 05 \mathrm{D} 6 \mathrm{FC} 50 \mathrm{~A} 73 \mathrm{~F} 3639 \mathrm{AB} 70 \mathrm{C} 19 \mathrm{~A} 18 \mathrm{E} 5234 \mathrm{~A} 172276 \mathrm{EE}$ 631E42A6A2CE5A28250424E4

$b=44808 \mathrm{~A} 33 \mathrm{D} 47780 \mathrm{EC} 13 \mathrm{CC} 721 \mathrm{C} 66605252 \mathrm{~A} 082008 \mathrm{AC} 5910272188$ $6382368 C C B 415802 \mathrm{~A} 4 \mathrm{E} 95 \mathrm{ACE}$

$r=$ 7FFFFFFFFFFFFFFFFFFFFFFFFFFFFFF09A007BD 63597 47C0A7181FC6DA9704EFB0C1

$P=$ (9F080369EA917727D1E1C709CC5BF2674AA7C79A2DFA5E5B 44 7F364F61690CD6DBAC05F5EFD， 558B8FB728CECBB9D0AA367 687E17D 6E97793769C71645AA1 68EEDCA3E2AE03D642B722572)

$Q=(\mathrm{F} 93 \mathrm{C} 3685 \mathrm{D} 5 \mathrm{E} 9 \mathrm{AB} 2 \mathrm{A0F} 7 \mathrm{C} 7 \mathrm{BD} 7 \mathrm{~F} 687 \mathrm{~A} 9 \mathrm{C} 4 \mathrm{E} 42 \mathrm{C} 10 \mathrm{C} 94 \mathrm{BD} 477 \mathrm{~F} 419$ E5C25B6E643B919F51CB3730B， 4C11A5F31FAD729F839F98D 34FB59D279C70A3126FFC3D5C1611F949340EEE12474A66263AC) $R=(\mathrm{FF} 00541676 \mathrm{FD} 0036 \mathrm{D} 12 \mathrm{COFEC} 3 \mathrm{~A} 1 \mathrm{~B} 8 \mathrm{D} 8 \mathrm{C} 692627 \mathrm{~F} 4 \mathrm{E} 8 \mathrm{DB} 62 \mathrm{~F} 45 \mathrm{~B}$ 708D9431C2E99F299984FD406, 37E09E68926A8157861A512 A86696A4A78A0F 0C15F 9EAC 4AECF 8BF 6D2B818284E8C3F 5853BD)

C300, $q=2^{20}, \mathbb{F}_{2^{20}}=\mathbb{F}_{2}[w] /\left(w^{20}+w^{10}+w^{9}+w^{7}+w^{6}+w^{5}+w^{4}+w+1\right)$ $f(u)=w^{327321} u^{31}+w^{349092} u^{30}+w^{995286} u^{28}+w^{930226} u^{24}+w^{602756} u^{16}+w^{602843}$ $h(u)=w^{687948} u^{15}+w^{946981} u^{14}+w^{169852} u^{12}+w^{811172} u^{8}+w^{458632}$ $D_{1}=\operatorname{div}\left(u^{15}+w^{173675} u^{14}+w^{1014246} u^{13}+w^{193959} u^{12}+w^{558539} u^{11}+w^{376720} u^{10}\right.$ $+w^{149697} u^{9}+w^{852573} u^{8}+w^{522198} u^{7}+w^{78372} u^{6}+w^{576415} u^{5}+w^{577000} u^{4}$ $+w^{1025691} u^{3}+w^{1030913} u^{2}+w^{224944} u+w^{165103}, w^{153473} u^{14}+w^{159391} u^{13}$ $+w^{624451} u^{12}+w^{540652} u^{11}+w^{1026818} u^{10}+w^{895055} u^{9}+w^{925553} u^{8}$ $+w^{700268} u^{7}+w^{449406} u^{6}+w^{518791} u^{5}+w^{428720} u^{4}+w^{109656} u^{3}+w^{362556} u^{2}$ $\left.+w^{818181} u+w^{438018}\right)$

$D_{2}=\operatorname{div}\left(u^{15}+w^{672767} u^{14}+w^{60108} u^{13}+w^{592469} u^{12}+w^{806912} u^{11}+w^{209094} u^{10}\right.$ $+w^{21555} u^{9}+w^{351715} u^{8}+w^{1006855} u^{7}+w^{553595} u^{6}+w^{115789} u^{5}+w^{940657} u^{4}$ $+w^{411255} u^{3}+w^{553233} u^{2}+w^{410382} u+w^{440174}, w^{456657} u^{14}+w^{165272} u^{13}$ $+w^{940178} u^{12}+w^{506617} u^{11}+w^{970890} u^{10}+w^{791679} u^{9}+w^{336652} u^{8}$ $+w^{568666} u^{7}+w^{937671} u^{6}+w^{23894} u^{5}+w^{617541} u^{4}+w^{400003} u^{3}+w^{792481} u^{2}$ $\left.+w^{36607} u+w^{409913}\right)$

$D_{3}=\operatorname{div}\left(u^{15}+w^{745174} u^{14}+w^{152075} u^{13}+w^{759312} u^{12}+w^{254997} u^{11}+w^{718088} u^{10}\right.$ $+w^{134849} u^{9}+w^{84810} u^{8}+w^{1017558} u^{7}+w^{909326} u^{6}+w^{549738} u^{5}+w^{64404} u^{4}$ $+w^{337345} u^{3}+w^{700483} u^{2}+w^{960561} u+w^{789792}, w^{163511} u^{14}+w^{370136} u^{13}$ $+w^{421951} u^{12}+w^{972631} u^{11}+w^{113274} u^{10}+w^{380219} u^{9}+w^{648060} u^{8}$ $+w^{564150} u^{7}+w^{642068} u^{6}+w^{819577} u^{5}+w^{633633} u^{4}+w^{662299} u^{3}+w^{542356} u^{2}$ $\left.+w^{473005} u+w^{146842}\right)$ 
E161-2, $N=161, \mathbb{F}_{2^{161}}=\mathbb{F}_{2}[z] /\left(z^{161}+z^{18}+1\right), \# \mathrm{E} 161\left(\mathbb{F}_{2^{161}}\right)=2 \cdot r$ $a=1$

$b=10000000000000000000000000000000000000062$

$r=$ FFFFFFFFFFFFFFFFFFFD5D528D29B3677A6BF15

$P=(1590236 \mathrm{CF}$ 8BCADEA57D2ABABDC7C918CD991F7FD1, 16019BB593140463E7C859426E4AD76188D4EA3C8)

$Q=(\mathrm{ACA} 7 \mathrm{~F} 2 \mathrm{D} 366101 \mathrm{~B} 27 \mathrm{~A} 6043551 \mathrm{C0B0D} 8 \mathrm{C} 60 \mathrm{~A} 32 \mathrm{ED} 98$, C15307FE705B5A5D638B40DF023D202E434D7D75)

E176, $N=176, \mathbb{F}_{2^{176}}=\mathbb{F}_{2}[z] /\left(z^{176}+z^{43}+z^{2}+z+1\right), \# \mathrm{E} 176\left(\mathbb{F}_{2176}\right)=65390 \cdot r$ $a=\mathrm{E} 4 \mathrm{E} 6 \mathrm{DB} 2995065 \mathrm{C} 407 \mathrm{D} 9 \mathrm{D} 39 \mathrm{~B} 8 \mathrm{D} 0967 \mathrm{~B} 96704 \mathrm{BA} 8 \mathrm{E} 9 \mathrm{C} 90 \mathrm{~B}$ $b=$ 5DDA 4 70ABE6414DE8EC133AE2 8E9BBD7FCECOAE0FFF 2 $r=10092537397 \mathrm{ECA} 4 \mathrm{~F} 6145799 \mathrm{D} 62 \mathrm{~B} 0 \mathrm{~A} 19 \mathrm{CE} 06 \mathrm{FE} 26 \mathrm{AD}$

$P=(96 \mathrm{E} 24$ 98B189AAD 4 55EC2431323B24E0603155C4EEE24, 2056F 497331B645ACAB8519F3F71099A71EBDD 7E2D06) $Q=(8 \mathrm{CE} 8805 \mathrm{EA} 1 \mathrm{D} 92 \mathrm{~A} 77975 \mathrm{~F} 69988 \mathrm{FF} 0 \mathrm{~B} 2 \mathrm{C} 99 \mathrm{~A} 3 \mathrm{C} 344 \mathrm{D} 469 \mathrm{D}$, 27A65C20DA08E0732D6327CF41E3C4B27AB9DB63706A)

E272, $N=272, \mathbb{F}_{2^{272}}=\mathbb{F}_{2}[z] /\left(z^{272}+z^{56}+z^{3}+z+1\right), \# \mathrm{E} 272\left(\mathbb{F}_{2272}\right)=65286 \cdot r$ $a=91 \mathrm{~A} 091 \mathrm{~F} 03 \mathrm{~B} 5 \mathrm{FBA} 4 \mathrm{AB} 2 \mathrm{CCF} 49 \mathrm{C} 4 \mathrm{EDD} 220 \mathrm{FB} 028712 \mathrm{D} 42 \mathrm{BE} 752 \mathrm{~B} 2 \mathrm{C} 4$ $0094 \mathrm{DBACDB} 586 \mathrm{FB} 20$

$b=7167 \mathrm{EFC} 92 \mathrm{BB} 2 \mathrm{E} 3 \mathrm{CE} 7 \mathrm{C} 8 \mathrm{AAAFF} 34 \mathrm{E} 12 \mathrm{~A} 9 \mathrm{C} 557003 \mathrm{D} 7 \mathrm{C} 73 \mathrm{~A} 6 \mathrm{FAF} 003$ F99F6CC8482E540F 7

$r=100 \mathrm{FAF}$ 51354E0E39E4892DF6E319C72C8161603FA45AA7B998A $167 \mathrm{~B} 8 \mathrm{~F} 1 \mathrm{E} 629521$

$P=$ (DE9DE5CD3B90447A20 6BEFE8167505CB7A28616DADABC639B 42 1DF763F 961D689DA9, $49831637686123445336 \mathrm{FE} 8 \mathrm{~B} 5$ 9FF 791C7 1CF 455823A4C375280A148B043DE7ECF17F)

$Q=(\mathrm{C} 9189420 \mathrm{~F} 828 \mathrm{C} 242771 \mathrm{E} 2 \mathrm{D} 64768930089 \mathrm{AB} 56 \mathrm{BA}$ E6D3A1DB2 94 AEDAD 60BAC9591E65, EB0123561D81715B23575A5DF3B13A771 C8521523C4EA853C0C4DF3294F70BF65AAA) 


\section{References}

1. ANSI X9.62, Public key cryptography for the financial services industry: the elliptic curve digital signature algorithm (ECDSA), 1999. 140, 164

2. S. Arita, 'Weil descent of elliptic curves over finite fields of characteristic three', $A d$ vances in Cryptology - Asiacrypt 2000, Lecture Notes in Comput. Sci. 1976 (Springer, 2000) 248-259. 146

3. E. ARTIN, 'Quadratische Körper im Gebiete der höheren Kongruenzen', Math. Z. 19 (1924) 207-246. 129

4. D. CAntor, 'Computing in the jacobian of a hyperelliptic curve', Math. Comp. 48 (1987) 95-101. 129

5. D. Coppersmith, A. Odlyzko and R. Schroeppel, 'Discrete logarithms in $G F(p)$ ', Algorithmica, 1 (1986) 1-15. 134

6. M. Daberkow, C. Fieker, J. Klüners, M. Pohst, K. Roegner, M. Schörnig and K. Wildanger, 'KANT V4', J. Symbolic Comput. 24 (1997) 267-283. 131

7. C. DiEM, 'A study on theoretical and practical aspects of Weil-restrictions of varieties', Ph.D. thesis, University of Essen, 2001. 146, 146

8. A. EngE and P. GAUDRY, 'A general framework for subexponential discrete logarithm algorithms', Acta Arith. 102 (2002) 83-103. 133

9. M. Fouquet, P. Gaudry and R. Harley, 'An extension of Satoh's algorithm and its implementation', J. Ramanujan Math. Soc. 15 (2000) 281-318. 140

10. G. Frey, 'Applications of arithmetical geometry to cryptographic constructions', Proceedings of the Fifth International Conference on Finite Fields and Applications (Springer, 2001) 128-161. 127

11. G. FreY and H. RücK, 'A remark concerning m-divisibility', Math. Comp. 62 (1994) 865-874. 127

12. S. Galbraith, 'Constructing isogenies between elliptic curves over finite fields', LMS J. Comput. Math. 2 (1999) 118-138;

http://www.lms.ac.uk/jcm/2/lms1998-010. 140

13. S. Galbraith and N. Smart, 'A cryptographic application of Weil descent', Codes and cryptography, Lecture Notes in Comput. Sci. 1746 (Springer, 1999) 191-200. 127

14. S. Galbraith, F. Hess and N. Smart, 'Extending the GHS Weil descent attack', Advances in Cryptology - Eurocrypt 2002, Lecture Notes in Comput. Sci. 2332 (Springer, 2002) 29-44. 140, 144, 148, 148

15. R. Gallant, R. Lambert and S. Vanstone, 'Improving the parallelized Pollard lambda search on anomalous binary curves', Math. Comp. 69 (2000) 1699-1705. 133,142

16. P. GAUDRY, 'An algorithm for solving the discrete log problem on hyperelliptic curves', Advances in Cryptology - Eurocrypt 2000, Lecture Notes in Comput. Sci. 1807 (Springer, 2000) 19-34. 133

17. P. Gaudry, F. Hess and N. Smart, 'Constructive and destructive facets of Weil descent on elliptic curves', J. Cryptology 15 (2002) 19-46. 127, 128, 130, 130, 130, 130, 131, $131,131,135,142,142$ 
18. F. HESS, KASH program for performing the GHS attack, 2000.

http://www.cs.bris.ac.uk/ nigel/weil_descent.html.131, 146, 164, 165

19. InTERnEt ENGINEERING TASK Force, 'The OAKLEY key determination protocol', IETF RFC 2412, November 1998; http: / / www. ietf.org/rfc/rfc2412. 139

20. M. Jacobson, A. Menezes and A. Stein, 'Solving elliptic curve discrete logarithm problems using Weil descent', J. Ramanujan Math. Soc. 16 (2001) 231-260. 134, $134,146,146,146$

21. A. JouX and R. LERCIER, 'Improvements on the general number field sieve for discrete logarithms in finite fields. A comparison with the Gaussian integer method', Math. Comp., to appear. 135

22. N. Koblitz, 'Hyperelliptic cryptosystems', J. Cryptology 1 (1989) 139-150. 129

23. A. Menezes and M. Qu, 'Analysis of the Weil descent attack of Gaudry, Hess and Smart', Topics in Cryptology - CT-RSA 2001, Lecture Notes in Comput. Sci. 2020 (Springer, 2001) 308-318. 128, 131, 131, 133

24. A. Menezes, T. Oкамото and S. Vanstone, 'Reducing elliptic curve logarithms to logarithms in a finite field', IEEE Trans. Inform. Theory 39 (1993) 1639-1646. 127

25. A. Menezes, P. van Oorschot and S. VAnstone, Handbook of applied cryptography (CRC Press, 1996). 164

26. P. van OORSCHOt and M. WIENER, 'Parallel collision search with cryptanalytic applications', J. Cryptology 12 (1999) 1-28. 127, 133

27. S. PAULUS and H. RÜCK, 'Real and imaginary quadratic representations of hyperelliptic function fields', Math. Comp. 68 (1999) 1233-1241. 130

28. S. Pohlig and M. Hellman, 'An improved algorithm for computing logarithms over $G F(p)$ and its cryptographic significance', IEEE Trans. Inform. Theory 24 (1978) 106-110. 127

29. J. Pollard, 'Monte Carlo methods for index computation mod p', Math. Comp. 32 (1978) 918-924. 127, 133

30. T. SATOH, 'The canonical lift of an ordinary elliptic curve over a finite field and its point counting', J. Ramanujan Math. Soc. 15 (2000) 247-270. 140

31. N. SMART, 'How secure are elliptic curves over composite extension fields?', Advances in Cryptology-Eurocrypt 2001, Lecture Notes in Comput. Sci. 2045 (Springer, 2001).

32. E. TESKE, 'Speeding up Pollard's rho method for computing discrete logarithms', Algorithmic number theory, Lecture Notes in Comput. Sci. 1423 (Springer, 1998) 541-554. 133

33. N. ThÉriault, 'Weil descent attack for Artin-Schreier curves', preprint, 2002. 131

34. M. Wiener and R. Zuccherato, 'Faster attacks on elliptic curve cryptosystems', Selected areas in cryptography, Lecture Notes in Comput. Sci. 1556 (Springer, 1999) 190-200. 133, 142

Markus Maurer

Alfred Menezes ajmeneze@uwaterloo.ca

Edlyn Teske eteske@uwaterloo.ca

Department of Combinatorics and Optimization

University of Waterloo, Canada 